\title{
An Experimentally Validated Modal Model Simulator for the Assessment of Different Blade Tip Timing Algorithms
}

DOI:

10.1016/j.ymssp.2019.106484

\section{Document Version}

Accepted author manuscript

Link to publication record in Manchester Research Explorer

\section{Citation for published version (APA):}

Mohamed, M., Bonello, P., \& Russhard, P. (2019). An Experimentally Validated Modal Model Simulator for the Assessment of Different Blade Tip Timing Algorithms. Mechanical Systems and Signal Processing.

https://doi.org/10.1016/j.ymssp.2019.106484

\section{Published in:}

Mechanical Systems and Signal Processing

\section{Citing this paper}

Please note that where the full-text provided on Manchester Research Explorer is the Author Accepted Manuscript or Proof version this may differ from the final Published version. If citing, it is advised that you check and use the publisher's definitive version.

\section{General rights}

Copyright and moral rights for the publications made accessible in the Research Explorer are retained by the authors and/or other copyright owners and it is a condition of accessing publications that users recognise and abide by the legal requirements associated with these rights.

\section{Takedown policy}

If you believe that this document breaches copyright please refer to the University of Manchester's Takedown Procedures [http://man.ac.uk/04Y6Bo] or contact uml.scholarlycommunications@manchester.ac.uk providing relevant details, so we can investigate your claim.

\section{OPEN ACCESS}




\section{An Experimentally Validated Modal Model Simulator for the}

2 Assessment of Different Blade Tip Timing Algorithms

3

4

5

6

7

8

9

10

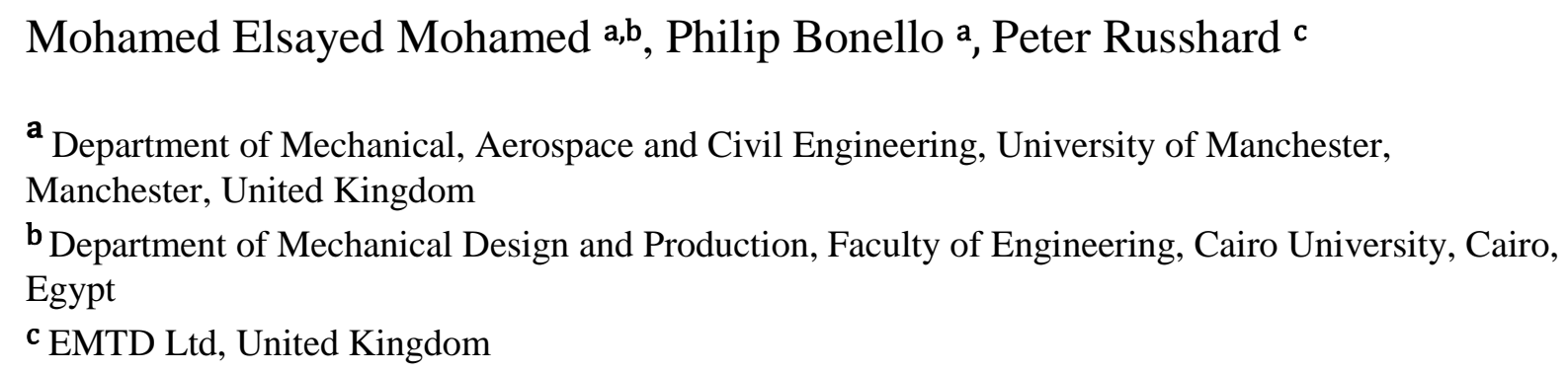

\section{ABSTRACT}

Blade Tip Timing (BTT) technology is concerned with the estimation of turbomachinery blade vibration. A BTT system comprises two parts: (a) measurement system for blade tip arrival times ("BTT data"); (b) the analysis algorithms. Simulators that generate BTT data play a key role in BTT development since they enable an assessment of the performance of the different BTT algorithms. Such assessments are not feasible with real engine data since they require controlled conditions and knowledge of the true tip vibration as the reference. Most simulators in the literature are based on a simple spring-mass-damper model and commercially available ones have no physical underpinning. This work presents a novel realistic simulator based on the experimentally validated Finite Element (FE) model of a bladed disk (blisk). Transformation to modal space enables the efficient generation of simulated BTT data regardless of the complexity of the blisk geometry. The simulator is then used in a first-time comparative study of three principal BTT algorithm methods (autoregressive, sine fitting, two-parameter plot) under various conditions involving synchronous and asynchronous excitations with both single and simultaneous frequencies. The study clarifies obscurities in the methods and demonstrates the applicability of each method to different BTT system scenarios.

\section{Keywords}

Blade Tip Timing; Finite Element Analysis; bladed assembly mechanics; measurement systems 
2 Turbomachinery blades are usually subjected to high stresses due to harsh operating 3 conditions such as speed, pressure, and temperature. Vibration measurements of bladed 4 assemblies (bladed disks or "blisks") play an important role in the testing phase, for 5 validating/refining mathematical models of effective designs that guarantee structural 6 integrity and reliability, as well as engine certification. An in-service blade vibration 7 measurement system can also form the basis of structural health monitoring system provided 8 it can survive the harsh operating conditions [1]. Blade tip timing (BTT) is considered to 9 provide an effective technique for both testing and monitoring of blades, and its applicability continues to be enhanced and extended through continuous development.

BTT is a non-contact non-intrusive measurement system comprising two main parts: (a) the measurement system; (b) the BTT data analysis algorithms. The BTT process is based on the detection of the arrival times of the tips of all blades at a number of circumferentially distributed probes (Fig. 1). If there is no vibration, the times would be equal to the expected values for rigid rotation of the blade. Different values would be obtained with the existence of vibration (or speed varying offsets like axial float). The difference between the measured and expected time of arrival (TOA) is used to calculate the blade tip displacement in the $x-y$ plane (the plane of rotation) as follows:

$d_{j}=\Omega R\left(t_{\mathrm{exp}, j}-t_{\mathrm{det}, j}\right)$

where $t_{\mathrm{exp}, j}$ and $t_{\mathrm{det}, j}$ are, respectively, the expected and actual (detected) TOAs of the blade tip at probe no. $j, j=1 \ldots N_{\mathrm{Pr}}, \Omega$ is the average rotational speed in $\mathrm{rad} / \mathrm{s}$ over one revolution 
1 and $R$ is the radius from the axis of rotation to the nominal sensing point on the blade tip.

2 The TOAs and the blade tip deflections computed from Eq. (1) are referred to as the "BTT

3 data". The uncertainty of this type of measurement depends on the precision of the measured

4 time and phase [2]. TOA measurements are under-sampled and include two types of noise:

5 "DC" and "AC". DC-noise appears as a steady-state offset in the data that is caused by

6 uncertainty in the position of the sensing point on the blade tip relative to the probe, resulting

7 from probe and/or blade positional errors, or from speed-dependent steady (non-oscillatory)

8 movements of the blisk (axial float, blade lean, and blade untwist) [3]. The authors presented

9 a method in [3-5] for determining the steady movements from BTT data, enabling the

10 identification of the true sensing point, and consequently improving the reliability of BTT vibration estimates. AC-noise arises from delays and errors in the sampling and acquisition instruments [6], and also from other significant sources such as blade buffeting [7]. The acquired data are analysed using one of the BTT algorithms to identify the amplitude, frequency, and phase of vibration. The identified tip vibration could then be used to estimate the stress at a chosen location using a pre-determined relationship based on a validated FE model of the blisk [8].

Different BTT algorithms (methods) have been developed in the last 20 years, starting with the Two-Parameter Plot (2PP) by Heath [9], and the sine fit method, also by Heath [10]. This was then followed by the auto-regressive (AR) methods [11-13], and more recently the multi-frequency sine fitting method introduced by Russhard in 2010 [1]. Other methods exist, but with little information, such as the circumferential Fourier Fit (CFF) method [14]. BTT methods can be categorised according to their applicability to different types of vibration to be identified - single frequency or multiple frequency vibration, for which a frequency component can be synchronous or asynchronous. Synchronous frequencies are 
1 integer engine orders (EOs, where EO is the ratio of the vibration frequency to the rotational

2 speed), whereas asynchronous frequencies are non-integer EOs.

3 The 2PP method was introduced by Heath in 1999 [9] and is used to find the EO of 4 synchronous single-frequency vibration. Claimed improvements to the identification 5 procedure for the EO using the $2 \mathrm{PP}$ method have been presented recently [15].

6 The auto-regressive (AR) class of techniques was introduced by Carrington et al. [11] in 2000 7 and developed further by Gallego Garrido et al. [12,13]. The most basic method in [11] was 8 valid only for the analysis of data resulting from a single synchronous excitation using 9 measurements of a single blade at a number of probes during a single revolution.

10 The upgraded class of AR methods developed by Gallego Garrido et al. [12][13] could be 11 used to analyse data from multiple (simultaneous) frequency excitations that can be synchronous and/or asynchronous. The methods in this class were designated in [12][13] as MAR/MGAR/MGARIV, from which further sub-varieties were derived (most notably those designated as MGAREP and MGARES), according to method of deriving the frequency. The applicability of each method was dependent on the parameter referred to as the "probe spacing on the resonance" (PSR) [12], which is defined as the engine order of the vibration to be identified multiplied by the angular spacing between the first and last probes:

$$
\operatorname{PSR}=\operatorname{EO}\left(N_{\mathrm{Pr}_{\mathrm{r}}}-1\right) \Delta \theta
$$

where $\Delta \theta$ is the probe angular spacing. Defining PSR as a percentage

$\% \mathrm{PSR}=100 \mathrm{EO}\left(N_{\mathrm{P}_{\mathrm{r}}}-1\right) \Delta \theta / 360^{\circ}$

the researchers in [12] showed that each method can be used within a specific range of \%PSR. Their conclusion was that MGARES is the most recommended AR method for 
1 systems subjected to either single or multiple excitations [13]. Hence, MGARES is the only

2 AR method considered in this paper.

3 Sine fitting algorithms involve fitting BTT data over one or more revolutions to a harmonic

4 function, or a combination of harmonic functions in case of multiple frequencies. Originally,

5 there were two standard algorithms of sine fitting of BTT data [16,17], both of which

6 applicable to a single frequency (or EO) - the three-parameter least square fit (first used in

7 [10]), and the four-parameter least square fit (first published in [16] and used recently in

8 [17]). The sine fitting method introduced by Russhard [1] is not restricted to a single EO

9 since the EOs are not determined as part of the fitting procedure, but pre-determined independently. In the case of vibration composed of integer EOs, the most convenient method to identify the relevant EOs is through the FE-derived Campbell diagram [1]. On the other hand, non-integer EOs are identified through a travelling wave method [1]. Russhard's work in [1] requires the data to be prepared before fitting, in order to separate the synchronous vibration (integer EOs) from the asynchronous. AC/DC noise components are also minimised. The method in [1] is therefore referred to here as Multi-frequency Sine Fitting with data Preparation (MSFP). This method is reported in [1] to be the current standard used in a leading aero-engine manufacturer, having replaced the earlier AR methods of [11] which revealed poor performance using real engine data due to the presence of noise. However, so far there has been no comparison between MSFP and the newer class of AR methods like MGARES. It should also be emphasised that, although the data preparation method in [1] was used for sine fitting, it could equally be applied to other BTT algorithms, as done for the first time in the present paper.

All the various BTT methods described above have been individually tested using either simulated and/or measured data [1,9-14]. Their performance and limits of applicability are mainly dependent on the following conditions: the number of frequencies contained in the 
1 vibration (single frequency/multiple frequencies); the type of excitation (synchronous and/or

2 asynchronous); the number of probes used; their angular spacing; the measurement noise

3 level [1,9-14]. What is lacking in the literature is an assessment of the principal BTT

4 methods (particularly MSFP and MGARES) with a view to establishing their applicability for

5 the aforementioned conditions. Such an assessment is not feasible with real engine data since

6 it needs to be made under controlled conditions and requires knowledge of the true tip

7 vibration as the reference. The assessment therefore needs to done using BTT data generated

8 from a simulator.

9 The BTT simulators presented in the literature have been typically based on simple mathematical models (discrete multi-degree-of-freedom spring-mass-damper systems) of the blisk $[6,11,18,19]$. Although such simulators can generate vibration with significant multifrequency content through the simultaneous excitation of multiple modes (which is why the simulator in [18] is described as a "multiple-modes simulator"), such modes are the product of a simplistic representation of the blisk resulting from the following assumptions: blade deflection limited to a single direction; elastic coupling between blades limited to adjacent blades only; the use of rigid supports for the connection between the blades and the disk. Moreover, many types of loadings and/or movements cannot be applied to such systems. It has been observed in [1] that while BTT algorithms may work well with data from such simplistic simulators, the same algorithms fail when applied to real data. On the other hand, while commercially available BTT measurement systems have the facility to generate simulated BTT data, these are not derived from a simulation of the actual mechanics of a blisk (they are instead synthesised from user-specified signal characteristics). A somewhat similar approach is used in the simulators presented in [20,21], where the BTT data are synthesised from actual measurements using the minimum number of probes. It is also noted 
1 that FE-based simulators used in previous works (Khayrton [22], Diamond [23]) were limited

2 to single mode resonance [24].

3 Hence, there is the need for a simulator that is based on a realistic model of the blisk, which

4 can accurately capture the complexity of the blisk design (and associated multi-mode

5 dynamics) while retaining computational efficiency. This latter quality is essential since the

6 process of determining a particular value of $t_{\mathrm{det}, j}$ in Eq. (1) with the proposed realistic

7 simulator involves multiple calculations in the integration process (including revisions in the

8 time step) to determine which of the nodes on a given blade tip coincides with the angular

9 and axial position of a given probe's centreline, and at what time.

The novel contributions of this paper are therefore as follows.

- A BTT data simulator based on the eigenvectors and eigenvalues of the FE model of a blisk. Transformation to modal coordinates is crucial for the operation of the simulator since the effective number of blisk modes required for accurate convergence of the vibration response is typically very much less than the number of degrees of freedom used in the FE model. Hence, the simulator can efficiently and accurately capture dynamic effects (e.g. the inter-blade coupling, blade-disk coupling). It can also allow the study of the effect of steady (non-oscillatory) movements (such as axial float, blade lean

- The application of the simulator to an experimentally validated FE model of a blisk in order to perform the aforementioned assessment of three principal BTT methods: 
1 The three BTT methods considered are presented in detail. During the course of this research

2 it was found that the MGARES two-frequency vibration expressions quoted in [12] gave the

3 wrong estimates. Hence, it was essential to repeat the derivation in [12] to find the correct

4 formulae. Also, the MGARES single-frequency vibration expressions, which are not given in

5 [12], are derived in this paper. The procedures for data preparation (section 3.1) and MSFP

6 (section 3.3) originate from the dissertation in [1] but have not been previously published in a

7 journal paper and are therefore presented in a different form. The experimental validation of

8 the modal parameters (eigenvectors and eigenvalues) of the blisk used in the assessment is

9 done using impact tests and FireWire cameras.

The simulator presented in this paper was first employed (but not presented in detail) by the authors in 2018 [3] in order to evaluate new algorithms for detecting steady movements. In the same year, independent researchers [24] presented a simulator that was also based on FEderived system modes. The work in [24] focused on tuning the simulator output to engine data, rather than assessing BTT algorithms. The literature review in [24] confirms the lack of multiple modes simulators that are based on realistic FE models. The similarities and differences between the simulator in [24] and the present one are detailed in section 2.2.

\section{SIMULATOR}

The simulator has three modules: (1) ANSYS module; (2) Simulink module; (3) Matlab module. The Matlab module interfaces with the other two modules and with the user. It allows the user to edit a pre-existing ANSYS input file for the FE analysis of a blisk according to the number of modes to be extracted. The free undamped modal analysis is then run in ANSYS and its output is saved to Excel files. This one-off computation for a given blisk is done for the non-rotating blisk in a fixed reference orientation (angular position). The eigenvalue analysis is then read into the Simulink module, which is based on the equations 
1 described in the following subsection and finds the expected and detected arrival times to

2 compute the blade tip displacements $d_{j}$ (as per Eq.(1)). These BTT data are then fed back to

3 the Matlab module for the subsequent processing.

$\mathbf{u}(t)=\left[\begin{array}{lllll}\cdots & x_{\mathrm{P}_{k}}(t) & y_{\mathrm{P}_{k}}(t) & z_{\mathrm{P}_{k}}(t) & \cdots\end{array}\right]^{\mathrm{T}}(3 K \times 1)$

14 where

$\mathbf{u}(t)=\mathbf{u}^{(\mathrm{rgd})}(t)+\Delta \mathbf{u}^{(\mathrm{def})}(t)+\Delta \mathbf{u}^{(\mathrm{off})}(t)$

15 In Eq. (5), $\mathbf{u}^{(\mathrm{rgd})}(t)$ represents the blade tip positions due to rigid rotation only (in the absence of deformation and any applied shifting), $\Delta \mathbf{u}^{(\mathrm{def})}(t)$ represents the response to the

17 excitation forces and $\Delta \mathbf{u}^{(\text {off })}(t)$ represents a prescribed steady offset that can vary with time.

18 This latter shift is optionally applied to simulate steady movements, which are a source of

\section{Fig. 2. HERE}

"DC noise" (Introduction). This was considered by the authors in [3-5] and is not considered here. The vector $\mathbf{u}^{(\mathrm{rgd})}(t)$ is given by 


$$
\mathbf{u}^{(\mathrm{rgd})}(t)=\left[\begin{array}{lllll}
\cdots & x_{\mathrm{P}_{k}}^{(\mathrm{rgd})}(t) & y_{\mathrm{P}_{k}}^{(\mathrm{rgd})}(t) & z_{\mathrm{P}_{k}}^{(\mathrm{rgd})}(t) & \cdots
\end{array}\right]^{\mathrm{T}}(3 K \times 1)
$$

1 Real tests are typically performed using accelerated (run-up) or decelerated (slow-down) 2 spinning of the rotor [19]. Hence, rigid rotation blade tip coordinates are calculated as 3 follows.

$$
x_{\mathrm{P}_{k}}^{(\mathrm{rgd})}(t)=R_{\mathrm{P}_{k}}^{(\mathrm{ref})} \cos \theta_{\mathrm{P}_{k}}^{(\mathrm{rgd})}(t), y_{\mathrm{P}_{k}}^{(\mathrm{rgd})}(t)=R_{\mathrm{P}_{k}}^{(\mathrm{ref})} \sin \theta_{\mathrm{P}_{k}}^{(\mathrm{rgd})}(t), z_{\mathrm{P}_{k}}^{(\mathrm{rgd})}(t)=z_{\mathrm{P}_{k}}^{(\mathrm{ref})}
$$

4 where

5 - The rigid rotation angular position $\theta_{\mathrm{P}_{k}}^{(\mathrm{rgd})}(t), 0 \leq \theta_{\mathrm{P}_{k}}^{(\mathrm{rgd})}<2 \pi$, is given by

$$
\theta_{\mathrm{P}_{k}}^{(\mathrm{rgd})}(t)=\theta_{\mathrm{P}_{k}}^{(\mathrm{ref})}+\Omega_{\mathrm{i}} t+\frac{\left(\Omega_{\mathrm{f}}-\Omega_{\mathrm{i}}\right)}{2 T_{\mathrm{s}}} t^{2}-2 \pi \cdot \text { floor }\left(\left\{\theta_{\mathrm{P}_{k}}^{(\mathrm{ref})}+\Omega_{\mathrm{i}} t+\frac{\left(\Omega_{\mathrm{f}}-\Omega_{\mathrm{i}}\right)}{2 T_{\mathrm{s}}} t^{2}\right\} /\{2 \pi\}\right)
$$

11 The term $\Delta \mathbf{u}^{(\mathrm{def})}(t)$ represents the oscillatory part of Eq. (5). Since it is superimposed on the 12 rigid rotation in Eq. (5) it is determined by considering the dynamic response of the non13 rotating blisk. The elements of $\Delta \mathbf{u}^{(\mathrm{def})}(t)$ are calculated from a transformation to modal 14 space using as basis functions the natural undamped modes of vibration of the non-rotating 


$$
\Delta \mathbf{u}^{(\mathrm{def})}(t)=\mathbf{H}_{\mathrm{P}} \mathbf{q}(t)
$$

1 where $\mathbf{H}_{\mathrm{P}}$ is the $3 K \times H$ matrix whose columns are the mass normalised eigenvectors

2 containing the modal displacements in the Cartesian degrees of freedom at the nodes $\mathrm{P}_{k}$

$3 \quad(k=1 \ldots K)$ of the blade tip:

$$
\mathbf{H}_{\mathrm{P}}=\left[\begin{array}{ccc}
\vdots & \cdots & \vdots \\
\phi_{x_{\mathrm{P}_{k}}}^{(1)} & \cdots & \phi_{x_{\mathrm{P}_{k}}}^{(H)} \\
\phi_{y_{\mathrm{P}_{k}}}^{(1)} & \cdots & \phi_{y_{\mathrm{P}_{k}}}^{(H)} \\
\phi_{\mathrm{P}_{k}}^{(1)} & \cdots & \phi_{\mathrm{P}_{\mathrm{P}_{k}}}^{(H)} \\
\vdots & \cdots & \vdots
\end{array}\right](3 K \times H)
$$

4 and $\mathbf{q}(t)$ is the $H \times 1$ vector of modal co-ordinates that are governed by the modal equations 5 of motion:

$$
\ddot{\mathbf{q}}(t)+\operatorname{diag}\left(\left[\cdots 2 \zeta_{r} \varpi_{r} \cdots\right]\right) \dot{\mathbf{q}}(t)+\mathbf{D q}(t)=\mathbf{H}_{\mathbf{f}}^{\mathrm{T}} \mathbf{f}(t)
$$

6 where:

- $\mathbf{f}(t)$ is the $3 N \times 1$ vector of dynamic excitation forces applied at nodes $Q_{n}(n=1 \ldots N)$ in the directions of the Cartesian degrees of freedom there:

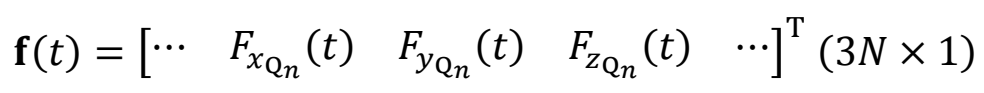

9

10

- $\quad \mathbf{H}_{\mathbf{f}}$ is the associated $3 N \times H$ matrix which is defined similarly to $\mathbf{H}_{\mathrm{P}}$ (Eq. (10)) but with subscript $\mathrm{P}_{k}$ replaced by $\mathrm{Q}_{n}$.

- $\mathbf{D}$ is the diagonal matrix containing the squares of the natural frequencies $\varpi_{r}(r=$ $1, \ldots, H)$, that correspond to the mode shapes (columns) in $\mathbf{H}_{\mathbf{P}}$ or $\mathbf{H}_{\mathbf{f}}$. 
2 - The modal damping matrix $\operatorname{diag}\left(\left[\cdots 2 \zeta_{r} \varpi_{r} \cdots\right]\right)$ is added to ensure decay of transients 3 when the excitation forces $\mathbf{f}(t)$ are user-prescribed time functions (as below).

4 The modal transformation using $H$ modes enables efficient solution of Eq. (11) regardless of 5 the complexity of the blisk since the effective number of modes required for accurate 6 convergence of Eq. (9) is typically much less than the number of degrees of freedom used in 7 the FE model of the blisk.

9 For an angular velocity sweep of duration $T_{\mathrm{s}}$, the excitation forces in $\mathbf{f}(t)$ (Eq. (11)) are assumed to be a superposition of $M$ chirp signals whose frequencies are fixed ratios (engine orders) $\mathrm{EO}_{m}, m=1 \ldots M$, of the time-varying angular speed, as described by the following formula [19] (using $F_{x_{Q_{n}}}(t)$ as an example):

$F_{\chi_{\mathrm{Q}_{n}}}(t)=\sum_{m=1}^{M} F_{x_{\mathrm{Q}_{n}}}^{(m)}(t)=\sum_{m=1}^{M} A_{x_{\mathrm{Q}_{n}}}^{(m)} \sin \left[\mathrm{EO}_{m}\left(\Omega_{\mathrm{i}} t+\left(\frac{\Omega_{\mathrm{f}}-\Omega_{\mathrm{i}}}{2 T_{\mathrm{S}}}\right) t^{2}\right)+\varphi_{x_{\mathrm{Q}_{n}}}^{(m)}\right]$

14 where $A_{x_{\mathrm{Q}_{n}}}^{(m)}$ and $\varphi_{x_{\mathrm{Q}_{n}}}^{(m)}$ are the force amplitude and phase respectively corresponding to 15 frequency component with engine order $\mathrm{EO}_{m}$. If a travelling wave model of blade excitation 16 is required, the phase angles are set according to the following formula [26] which is a 17 corrected version of the one in [19]:

$\varphi_{x_{\mathrm{Q}_{n}}}^{(m)}=2 \pi i N_{\mathrm{ND}} / N_{\mathrm{b}} \quad i=0,1, \ldots \ldots,\left(N_{\mathrm{b}}-1\right)$

18 where $i$ is the number of the blade to which node $\mathrm{Q}_{n}$ belongs, and $N_{\mathrm{ND}}$ is the number of nodal diameters. It is important to note that the forces $\mathbf{f}(t)$ are applied to the non-rotating blisk and their responses superimposed on the rigid rotation displacement (Eq. (5)) i.e. the coordinate 
1 system used in modal Eq. (11) and forces $\mathbf{f}(t)$ in Eq. (12) is at a fixed angular position

2 relative to the spinning blisk. The force at a given node $Q_{n}$ has multiple frequencies which

3 vary with the rotational speed $\Omega(t)$. For constant $\Omega(t)$ (i.e. $\Omega_{\mathrm{f}}=\Omega_{\mathrm{i}}$ ), Eq. (13) reduces to the

4 conventional Fourier series used to represent arbitrary periodic excitation on a given blade

5 [27]. For $\Omega_{\mathrm{f}} \neq \Omega_{\mathrm{i}}$, Eq. (13) is a modified Fourier series, wherein the basis functions $F_{x_{\mathrm{Q}_{n}}}^{(m)}(t)$

6 are swept harmonic functions. In either case, the circular frequencies are $\mathrm{EO}_{m}$.

$7\left(\Omega_{\mathrm{i}}+\left(\frac{\Omega_{\mathrm{f}}-\Omega_{\mathrm{i}}}{T_{\mathrm{s}}}\right) t\right)\left(=\mathrm{EO}_{m} \Omega(t)\right)$ (by differentiation of term in [ ]). If the $\mathrm{EO}_{m}$ are all

8 integers, the fundamental frequency of the excitation will be $\Omega(t)$. In any case, the

9 frequencies $\mathrm{EO}_{m} \Omega(t)$ of interest are only those coinciding with one of the natural

10 frequencies, as per Campbell diagram [1].

12 Let $\left(x_{j}, y_{j}, z_{j}\right)$ be the coordinates of the tip centre of probe no. $j\left(j=1 \ldots N_{\mathrm{Pr}}\right)$. The nominal sensing point on a given blade for probe no. $j$ is denoted by $\mathrm{P}_{k_{\mathrm{nom}, j}}$, which corresponds to the node $\mathrm{P}_{k}$ on the blade tip that, in the fixed reference orientation of the undeformed and unshifted blisk, has the closest axial position to that of the tip centre of probe no. $j$ :

$$
\left|z_{\mathrm{P}_{k_{\text {nom }}, j}}^{(\mathrm{ref})}-z_{j}\right|=\min \left(\left[\left|z_{\mathrm{P}_{1}}^{(\mathrm{ref})}-z_{j}\right| \cdots\left|z_{\mathrm{P}_{k}}^{(\mathrm{ref})}-z_{j}\right| \quad \cdots\left|z_{\mathrm{P}_{K}}^{(\mathrm{ref})}-z_{j}\right|\right]\right)
$$

17 It should be noted that for a given blade, $\mathrm{P}_{k_{\text {nom }, j}}$ may be different for different probes since these may be mounted at different axial locations.

Letting $\theta_{j}, 0 \leq \theta_{j}<2 \pi$, be the angular position in the $x-y$ plane of probe no. $j$, the expected arrival time of the blade at probe no. $j, t_{\mathrm{exp}, j}$, is given by solving the quadratic equation: 
$\theta_{\mathrm{P}_{k_{\mathrm{nom}}, j}}^{(\mathrm{rgd})}(t)=\theta_{j}$, for $t=t_{\mathrm{exp}, j}$

1 where the left hand side of Eq. (16) is given by the right hand side of Eq. (8) with the

2 appropriate value of $k\left(k_{\text {nom,j }}\right)$ identified from eqn. (15).

3 The actual (detected) arrival times at probe no. $j$ corresponding to each node $\mathrm{P}_{k}$ on the blade 4 tip are given by the times $t=t_{\theta_{j}, \mathrm{P}_{k}}$ for which

$$
\theta_{\mathrm{P}_{k}}\left(x_{\mathrm{P}_{k}}(t), y_{\mathrm{P}_{k}}(t)\right)=\theta_{j}
$$

5 where $\theta_{\mathrm{P}_{k}}$ is the actual angular position of $\mathrm{P}_{k}$ in the plane of rotation ( $x-y$ plane), as per

6 elements $x_{\mathrm{P}_{k}}(t), y_{\mathrm{P}_{k}}(t)$ in $\mathbf{u}(t)$ in Eq. (4) computed according to equations (5) to (11).

7 The modal equations (11) are integrated using the Matlab function ode45 via Simulink. The 8 use of Simulink was essential in order to benefit from the in-built function block Hit 9 Crossing, which was also used in the spring-mass simulator of [19]. In the present case Hit 10 Crossing solves Eq. (17) by comparing $\theta_{\mathrm{P}_{k}}$ to $\theta_{j}$ and can force the integrator to change the 11 time steps until it finds the required crossing time. This process is done at each time step for 12 each of the nodes on the tip of a blade. Of these $K$ times $\left(t=t_{\theta_{j}, \mathrm{P}_{k}}, k=1 \ldots K\right)$, the one that 13 is taken to be the detected blade arrival time at probe no. $j, t=t_{\mathrm{det}, j}$, is the one 14 corresponding to the node $\mathrm{P}_{k_{\mathrm{det}}, j}$ which is the node $\mathrm{P}_{k}$ on the blade tip for which the axial 15 coordinate at instant $t=t_{\theta_{j}, \mathrm{P}_{k}}$ is closest to that of the centre of probe no. $j$ as shown in Fig. 2 and expressed mathematically below: 


$$
\begin{aligned}
& \left|z_{\mathrm{P}_{k_{\mathrm{det}}, j}}\left(t_{\theta_{j}, \mathrm{P}_{k_{\mathrm{det}}, j}}\right)-z_{j}\right| \\
& =\min \left(\left[\left|z_{\mathrm{P}_{1}}\left(t_{\theta_{j}, \mathrm{P}_{1}}\right)-z_{j}\right| \quad \cdots\left|z_{\mathrm{P}_{k}}\left(t_{\theta_{j}, \mathrm{P}_{k}}\right)-z_{j}\right| \cdots\left|z_{\mathrm{P}_{K}}\left(t_{\theta_{j}, \mathrm{P}_{K}}\right)-z_{j}\right|\right]\right)
\end{aligned}
$$

1 Equation (18) is solved using a separate custom-written function block.

2 Having determined $t_{\mathrm{det}, j}$ and $t_{\mathrm{exp}, j}$, the instantaneous tip displacement when passing probe

3 no. $j, d_{j}$, is calculated from Eq. (1), where $\Omega$ is the average angular velocity over the

4 revolution considered and $R=R_{\mathrm{P}_{k_{\mathrm{nom}}}}^{(\mathrm{ref})}$, where $\mathrm{P}_{k_{\text {nom }}}$ is the nominal sensing point for probe $5 \quad$ no. $j$ as defined by Eq. (15).

6 The process described by equations (15)-(18) refers to a given blade passing a given probe 7 no. $j$ and therefore needs to be repeated for all probes $j=1 \ldots N_{\operatorname{Pr}}$ over each revolution. For 8 the work in section 5, it is sufficient to have BTT data from one blade as detected by all 9 probes, except when determining a non-integer EO by the travelling wave method (as part of the MSFP, section 3.3), since this additionally requires data from all blades as detected by two probes.

14 Fig. 3 shows a flow schematic of the simulator, summarising the process described above for a given blade and given probe. The final stage is to corrupt the BTT data by adding AC noise, which is taken to be Gaussian white noise. This is generated using the standard normal

17 distribution with zero mean and unit variance and then normalized and multiplied by a scaling factor $\gamma$ to achieve the desired noise-to-signal ratio (NSR). NSR is defined as the ratio 
1 between the root mean square (RMS) of the noise values to the RMS of the pure BTT

2 displacements [11]:

$$
\mathrm{NSR}=\frac{\text { RMS(Normalized Noise }) * \gamma}{\sqrt{\sum_{n=1}^{N_{\text {revs }}} \sum_{j=1}^{N_{\operatorname{Pr}}}\left({ }^{n} d_{j}\right)^{2} /\left(N_{\text {revs }} N_{\mathrm{Pr}}\right)}}
$$

4 where ${ }^{n} d_{j}$ is the displacement of a given blade observed by probe no. $j$ during revolution no. $5 \quad n$ and the denominator of Eq. (19) is the global RMS of these displacements. The nominal 6 value of NSR in BTT is $10 \%$ [12].

\subsection{Discussion on Simulator Capabilities}

8 With regard to the simulator [24] referred to at the end of section 1, developed at the same 9 time by other researchers, the following observations are made.

a) A subset of nominal system modes of a compressor rotor was used in [24]. The present work focuses on the blisk modes, but the modes of the wider system can be considered if available.

b) The solver in [24] used an implicit integration scheme with fixed time step. The present simulator uses automatic variable step size control to maintain the prescribed error tolerance; Simulink offers a wide choice of explicit/implicit solvers.

c) The detected arrival time at probe no. $j$ corresponding to a given point in a given revolution was determined in [24] simply by interpolation. In other words, with reference to equation (17), the TOA of $\mathrm{P}_{k}$ was determined in [24] by interpolating between two integration time values $t_{1}, t_{2}$ for which

$$
\theta_{\mathrm{P}_{k}}\left(x_{\mathrm{P}_{k}}\left(t_{1}\right), y_{\mathrm{P}_{k}}\left(t_{1}\right)\right)<\theta_{j}<\theta_{\mathrm{P}_{k}}\left(x_{\mathrm{P}_{k}}\left(t_{2}\right), y_{\mathrm{P}_{k}}\left(t_{2}\right)\right)
$$


i.e. the algorithm did not alter the integration time through a function like Hit Crossing to find the "precise" crossing time, as explained under Eq. (17) and in Fig. 3. This simple approach could be used in the present simulator and will result in considerable time saving. However, there are concerns of accuracy. An investigation into the error incurred in using simple interpolation $v s$ the more accurate Hit Crossing approach would be a useful future research work.

d) The simulator in [24] allowed the following optional inputs: noise; motion-dependent aerodynamic forces; mistuning effect; probe position error (PPE); blade static deflection; blade position error (BPE). The present work can accommodate noise, aerodynamic, and mistuning effects in the same way. PPE and BPE affect $t_{\text {exp, } j}[4]-$ thus, angular or axial PPE can be considered by adding error terms $\Delta \theta_{j}, \Delta z_{j}$ to Eqs. (17), (18) respectively. Blade static deflection and other steady shifts can be accommodated through the term $\Delta \mathbf{u}^{\text {(off) }}(t)$ in Eq. (5), as done by authors in [4].

e) The simulator in [24] also accommodated gyroscopic/Coriolis effects as an optional input, but these were based on a constant gyroscopic matrix (obtained from FE) applicable to a single speed only. The corresponding natural frequencies used in the modal equations included rotational effects but were only applicable for a single speed. Hence, the variation in rotational speed considered in [24] was only around 10\%. Such an approach is not suitable for the present work since the speed varies over a much wider range. Hence, the present version of the simulator neglects gyroscopic effects, centrifugal stress stiffening/spin softening [28]. This is justified by the use of a lightweight aluminium blisk in the studies of sections 4 and 5. A future version of the simulator is under development which shall accommodate rotational inertia effects without speed restriction by considering them as "external" excitations in Eq. (11) so as to retain the non-rotational modes as basis functions. 
2 The general form of the blade tip displacement $d_{j}$ measured at probe no. $j$ prior to data 3 preparation is assumed to follow the following form

$$
d_{j}=P_{j}+\sum_{m=1}^{M}\left(a_{1 m} \sin \left(\omega_{m} t_{j}\right)+a_{2 m} \cos \left(\omega_{m} t_{j}\right)\right)+\text { noise }
$$

4 where $\omega_{m}=\mathrm{EO}_{m} \Omega$ is a generic frequency component. $t_{j}$ represents the sampling time 5 according to the assumptions made in the BTT algorithm used. $P_{j}$ is the steady offset 6 resulting from positional errors in the probes and/or blades, and blade steady movements.

\subsection{Data Preparation Process}

8 With reference to Eq. (21), the raw BTT data may include both synchronous (integral EOs) 9 and asynchronous (non-integral EOs) components. The aim of the data preparation is to separate both types and to minimise the offset $P_{j}$ and the noise. The process introduced by Russhard [1] will be illustrated with respect to the examples shown in Figs. 4(a,b), 5(a,b), wherein the raw data refers to the tip displacement of a given blade observed by one of six probes over a large number of revolutions as the rotational speed was swept in order to traverse a blisk resonance. The data in Figs. 4, 5 pertains to Test no.5 (Table 2) in section 5. The excitation was such that the vibration response had two EOs: 2 and 3.6. The raw data was generated by the simulator of section 2 and corrupted with $20 \%$ NSR. It should be noted that the strong oscillatory profile in Figs. $4(a), 5(a, b)$ is caused by the non-integer EO, not the noise. 
1 In order to extract only the synchronous vibration response, both non-integral and noise

2 signals are reduced using the Savitsky-Golay smoothing filters [29] as shown in Fig. 4(a).

3 Savitsky-Golay filters work on minimizing the least squares error in fitting a polynomial to

4 frames of noisy data. The smoothing in Fig. 4(a) was done using Matlab function sgolayfilt

5 [30] with polynomial order of 3 and frame size of 51.

6 The next step is to minimise any offset $\left(P_{j}\right)$ in the data. This is done by dividing the

7 smoothed data into a large number of segments (buffers) each of length $N_{\text {buff }}$ points (each

8 point corresponding to a revolution). For the data in Fig. 4(a), the buffer length is 10 points.

9 If the arithmetic average of each buffer is calculated and subtracted from the smoothed data,

10 not only is the data offset $P_{j}$ minimised, but a large portion of the integral EO data is removed

11 (since for integer $\mathrm{EO}_{m}$ and gradual variations in rotational speed, the difference in $d_{j}$ from

12 one revolution to the next is very small). Hence, to preserve the integer EO data, a mask is

13 placed on the resonance regions. If $b_{k}$ is the average of buffer no. $k$ and the masked region

$14 \mathrm{AB}$ is bounded by buffer nos. $k_{\mathrm{A}}$ and $k_{\mathrm{B}}$, then:

- for $k<k_{\mathrm{A}}, k>k_{\mathrm{B}}$ (buffers outside $\mathrm{AB}$ ) - $b_{k}$ is calculated by arithmetic average of data in buffer.

- for $k_{\mathrm{A}}<k<k_{\mathrm{B}}$ (buffers inside $\mathrm{AB}$ ) - $b_{k}$ is replaced by a modified "average" $\hat{b}_{k}$ calculated by linear interpolation between $b_{k_{\mathrm{A}}}$ and $b_{k_{\mathrm{B}}}$.

This process is illustrated in Fig. 4(a).

The resonance regions to be masked are identified by calculating the coherence of the probe displacement data on a revolution-by-revolution basis using the cross correlation factor $X_{r}$ of Carrington [6]. 


$$
X_{r}=\frac{2 \sum_{j=1}^{N_{\operatorname{Pr}}}\left({ }^{n} d_{j}\right)\left({ }^{n+1} d_{j}\right)}{\sum_{j=1}^{N_{\operatorname{Pr}}}\left({ }^{n} d_{j}\right)^{2}+\sum_{j=1}^{N_{\operatorname{Pr}}}\left({ }^{n+1} d_{j}\right)^{2}}
$$

1 where ${ }^{n} d_{j}$ was defined below equation (19). According to [1], for amplitudes in the

2 resonance region of at least $0.1 \mathrm{~mm}$, it is experimentally shown that $X_{r}^{2}$ should be close to $3 \quad 0.99$.

4 The buffer averages/modified averages $b_{k}, \hat{b}_{k}$ in Fig. 4(a) are then subtracted from the 5 corresponding data points. The resulting smoothed data with offset minimised is shown in 6 Fig. 4(b) and this data represents the synchronous 2 EO component of the original two7 frequency noisy data.

8

9 It should be noted that, to determine whether or not the above procedure should be applied to the original data, it is necessary to refer to the Campbell diagram (e.g. Fig. 6) to identify any resonant integer EOs within the speed range considered. Once the separated integer EO data is processed by the BTT algorithm, the quality of fit of the BTT results is evaluated as per section 3.3. Such a priori information is generally essential to target the correct responses [1] especially when using MSFP to calculate the amplitude/phase of integer EOs (section 3.3).

Fig. 6 HERE

The extraction of the asynchronous components involves the removal of the integral and steady-offset component from the unfiltered data (Eq. (21)) by subtracting the buffer averages $b_{k}$ as in the previous section but without applying the masks over the resonance region. This is illustrated in Fig. 5(a). The resulting data in Fig. 5(b) represents the non- 
1 synchronous component (3.6 EO) of the raw data plus the added noise. It is noted that noise

2 cannot be separated from the non-integer EO. Hence, the uncertainty from noise is a more

3 significant factor in the BTT estimation of non-integer EOs than integer EOs.

5 After the above process, Eq. (21) is revised as follows:

$$
\begin{aligned}
d_{j}=a_{0}+\sum_{m=1}^{M}\left(a_{1 m} \sin \left(\omega_{m} t_{j}\right)+a_{2 m} \cos \left(\omega_{m} t_{j}\right)\right) \\
=a_{0}+\sum_{m=1}^{M}\left(a_{1 m} \sin \left(\mathrm{EO}_{m} \Omega t_{j}\right)+a_{2 m} \cos \left(\mathrm{EO}_{m} \Omega t_{j}\right)\right)
\end{aligned}
$$

6 where the $\mathrm{EO}_{m}$ 's are either all integers or all non-integers and $a_{0}$ is the corresponding

7 residual offset. In the case of non-integer EOs, $a_{0}$ is mainly due to the noise. It is noted that

$8 d_{j}$ in Eq. (23) refers to the prepared data, whereas $d_{j}$ in Eq. (21) is the raw data (the notation

9 is retained to avoid complication). Also, it is noted that MGARES (section 3.2) does not require separation of synchronous from non-synchronous components, unlike MSFP (section

3.3). However, the data is prepared in the same way of this section for all methods to ensure a fair comparison.

For a constant rotational speed $\Omega$, the once-per-revolution sampling times are given by $t_{j}=t_{j, k=1}+(k-1) 2 \pi / \Omega, \quad k=1,2, \ldots$ and so, the term $\mathrm{EO}_{m} \Omega t_{j}=\mathrm{EO}_{m} \Omega\left\{t_{j, k=1}+\right.$ $(k-1) 2 \pi / \Omega\}$ always represents the same angle $\mathrm{EO}_{m} \Omega t_{j, k=1}$ provided $\mathrm{EO}_{m}$ is a fixed integer. Hence, Eq. (23) can then be rewritten in terms of the fixed probe angular position $\theta_{j}=\Omega t_{j, k=1}$ as follows: 
$d_{j}=a_{0}+\sum_{m=1}^{M}\left(a_{1 m} \sin \left(\mathrm{EO}_{m} \theta_{j}\right)+a_{2 m} \cos \left(\mathrm{EO}_{m} \theta_{j}\right)\right)$

1 The representation of Eq. (24) is also valid if the rotational speed $\Omega$ is assumed to be

2 approximately constant over one revolution, while varying gradually over time.

3 The format of Eq. (24) is used by MSFP (section 3.3) and the 2PP (section 3.4). The 2PP

4 relies on the format of Eq. (24) (with single frequency term) to identify the EO. Hence, the

$52 \mathrm{PP}$ is limited to synchronous vibration (integer EOs). However, as shown in section 3.3, the

6 identification of the EOs in the MSFP is independent of Eq. (24), which is only used to

7 identify the coefficients $a_{0}, a_{1 m}, a_{2 m}$. This explains the use of Eq. (24) with non-integer

8 EOs in the MSFP method with negligible error in [1], as also confirmed in the present paper.

9

$t_{j-m}=t_{j}-m \Delta t, m=1,2, \ldots$

$$
\Delta t=\Delta \theta / \Omega
$$

17 It is assumed that response $d_{j}$ at the different probes follow the linear-prediction auto-

$$
d_{j}=a_{0}+\sum_{m=1}^{M} A_{m} \sin \left(\omega_{m} t_{j}+\psi_{m}\right)
$$

It is assumed that the frequencies $\omega_{m}=\mathrm{EO}_{m} \Omega$ are constant, which means that the rotational speed $\Omega$ is assumed to be approximately constant, even when multiple revolutions are considered. It is also assumed that the probes are equally spaced at angular intervals $\Delta \theta$ so that the times $t_{j}$ assumed in Eq. (25) are given by:

$t_{j-m}=t_{j}-m \Delta t, m=1,2, \ldots$ regressive equation for a system of multi-DOF with $M$ simultaneous excitations 


$$
d_{j}=c_{0}-\sum_{m=1}^{2 M} c_{m} d_{j-m}
$$

1 where $c_{0}$ is the constant offset, and $c_{m}$ is an AR coefficient. The equations in (27) can be

2 rewritten for a single revolution as in Multiple Auto-Regressive method (MAR) using a 3 matrix representation as follows

$$
\begin{aligned}
& {\left[\begin{array}{c}
d_{2 M+1} \\
d_{2 M+2} \\
\vdots \\
d_{N_{\mathrm{Pr}}}
\end{array}\right]=\left[\begin{array}{ccccc}
-d_{2 M} & -d_{2 M-1} & \cdots & -d_{1} & 1 \\
-d_{2 M+1} & -d_{2 M} & \cdots & -d_{2} & 1 \\
\vdots & \vdots & \ddots & \vdots & \vdots \\
-d_{N_{\mathrm{Pr}}-1} & -d_{N_{\mathrm{Pr}}-2} & \cdots & -d_{N_{\mathrm{Pr}}-2 M} & 1
\end{array}\right]\left[\begin{array}{c}
c_{1} \\
c_{2} \\
\vdots \\
c_{2 M} \\
c_{0}
\end{array}\right]} \\
& \text { Or } \quad \mathbf{d}=\mathbf{B c}
\end{aligned}
$$

4 The above system of equations can be solved as

$\mathbf{c}=\left(\mathbf{B}^{\mathrm{T}} \mathbf{B}\right)^{-\mathbf{1}} \mathbf{B}^{\mathrm{T}} \mathbf{d}$

5 The minimum number of probes $N_{\mathrm{Pr}_{\text {min }}}$ required by this method is

$$
N_{\operatorname{Pr}_{\text {min }}}=4 M+1
$$

6 The method has been extended to use observations from a multiple revolutions $N_{\text {revs }}$ for the 7 same blade:

$$
\left[\begin{array}{c}
{ }^{1} \mathbf{d} \\
\vdots \\
N_{\text {revs }} \mathbf{d}
\end{array}\right]=\left[\begin{array}{c}
{ }^{1} \mathbf{B} \\
\vdots \\
N_{\text {revs }} \mathbf{B}
\end{array}\right] \mathbf{C}
$$

8 where the left-hand superscript denotes data taken at a given revolution. Use of the multiple 9 revolution Eqn. (32) instead of the single revolution Eq. (28) ("MAR") is referred in [12] to 10 as "MGAR" where the letter "G" stands for "global". 
1 The number of unknowns in $\mathbf{c}$ can be reduced by substituting the expression (25) and

2 manipulating trigonometric expressions, resulting in the following reduced system:

$$
\left[\begin{array}{c}
{ }^{1} \hat{\mathbf{d}} \\
\vdots \\
N_{\text {revs }} \hat{\mathbf{d}}
\end{array}\right]=\left[\begin{array}{c}
{ }^{1} \widehat{\mathbf{B}} \\
\vdots \\
N_{\text {revs }} \mathbf{B}
\end{array}\right] \mathbf{c}_{\text {red }}
$$

4 MGARES involves the identification of the constants $c_{0}, c_{1}, c_{2} \ldots$ in Eq. (33)) and the

5 determination of the frequencies $\omega_{m}$ in Eq. (25) through direct (symbolic) expressions for the

6 frequencies in terms of some of these constants. The remainder of this section presents

7 MGARES for the case of two-frequency excitation and the case of single-frequency

8 excitation wherein new expressions for frequency are derived in either case.

9 Substituting the expressions for $d_{j}$ and $d_{j-m}$ from Eq. (25) and (26) into Eq. (27) and 10 isolating the sine and cosine terms for the case $M=2$ yields:

$$
\begin{aligned}
& A_{1} \sin \left(\omega_{1} t_{j}+\psi_{1}\right) \times\left(1+c_{1} \cos \left(\omega_{1} \Delta t\right)+c_{2} \cos \left(\omega_{1} 2 \Delta t\right)+c_{3} \cos \left(\omega_{1} 3 \Delta t\right)+c_{4} \cos \left(\omega_{1} 4 \Delta t\right)\right)=0 \\
& A_{1} \cos \left(\omega_{1} t_{j}+\psi_{1}\right) \times\left(c_{1} \sin \left(\omega_{1} \Delta t\right)+c_{2} \sin \left(\omega_{1} 2 \Delta t\right)+c_{3} \sin \left(\omega_{1} 3 \Delta t\right)+c_{4} \sin \left(\omega_{1} 4 \Delta t\right)\right)=0 \\
& A_{2} \sin \left(\omega_{2} t_{j}+\psi_{2}\right) \times\left(1+c_{1} \cos \left(\omega_{2} \Delta t\right)+c_{2} \cos \left(\omega_{2} 2 \Delta t\right)+c_{3} \cos \left(\omega_{2} 3 \Delta t\right)+c_{4} \cos \left(\omega_{2} 4 \Delta t\right)\right)=0 \\
& A_{2} \cos \left(\omega_{2} t_{j}+\psi_{1}\right) \times\left(c_{1} \sin \left(\omega_{2} \Delta t\right)+c_{2} \sin \left(\omega_{2} 2 \Delta t\right)+c_{3} \sin \left(\omega_{2} 3 \Delta t\right)+c_{4} \sin \left(\omega_{2} 4 \Delta t\right)\right)=0
\end{aligned}
$$

11 The equations in (34) are solved to give the following expressions:

$$
\begin{aligned}
& c_{1}=-2\left(\cos \left(\omega_{1} \Delta t\right)+\cos \left(\omega_{2} \Delta t\right)\right) \\
& c_{2}=2\left(1+2 \cos \left(\omega_{1} \Delta t\right) \cos \left(\omega_{2} \Delta t\right)\right) \\
& c_{3}=-2\left(\cos \left(\omega_{1} \Delta t\right)+\cos \left(\omega_{2} \Delta t\right)\right) \\
& c_{4}=1
\end{aligned}
$$


1 It is clear from (35) that $c_{1}=c_{3}$ and the value of $c_{4}$ is known. Hence, for two frequencies,

2 the reduced unknowns vector in Eq. (33) is $\mathbf{c}_{\mathrm{red}}=\left[\begin{array}{lll}c_{1} & c_{2} & c_{0}\end{array}\right]^{\mathrm{T}}$ and the corresponding 3 reduced system of equations (33) will be:

$$
\left[\begin{array}{c}
{ }^{1} d_{5}+{ }^{1} d_{1} \\
{ }^{1} d_{6}+{ }^{1} d_{2} \\
{ }^{1} d_{7}+{ }^{1} d_{3} \\
\vdots \\
{ }_{N_{\text {revs }}} d_{5}+{ }^{N_{\text {revs }}} d_{1} \\
{ }^{N_{\text {revs }}} d_{6}+{ }^{N_{\text {revs }}} d_{2} \\
{ }^{N_{\text {revs }}} d_{7}+{ }^{N_{\text {revs }}} d_{3}
\end{array}\right]=\left[\begin{array}{ccc}
-\left({ }^{1} d_{4}+{ }^{1} d_{2}\right) & -\left({ }^{1} d_{3}\right) & 1 \\
-\left({ }^{1} d_{5}+{ }^{1} d_{3}\right) & -\left({ }^{1} d_{4}\right) & 1 \\
-\left({ }^{1} d_{6}+{ }^{1} d_{4}\right) & -\left({ }^{1} d_{5}\right) & 1 \\
\vdots & \vdots & \vdots \\
-\left({ }^{N_{\text {revs }}} d_{4}+{ }^{N_{\text {revs }}} d_{2}\right) & -\left({ }^{N_{\text {revs }}} d_{3}\right) & 1 \\
-\left({ }^{N_{\text {revs }}} d_{5}+{ }^{N_{\text {revs }}} d_{3}\right) & -\left({ }^{N_{\text {revs }}} d_{4}\right) & 1 \\
-\left({ }^{N_{\text {revs }}} d_{6}+{ }^{N_{\text {revs }}} d_{4}\right) & -\left({ }^{N_{\text {revs }}} d_{5}\right) & 1
\end{array}\right]\left[\begin{array}{l}
c_{1} \\
c_{2} \\
c_{0}
\end{array}\right]
$$

4 Thus the minimum required number of probes in Eq. (36) has been reduced to 7 instead of 9 5 (for the unreduced case in Eq. (32)). By solving the first two equations in (35), a solution for 6 each frequency can be obtained by the following equations.

$$
\begin{aligned}
& \omega_{1}=\cos ^{-1}\left(\frac{1}{4}\left[-c_{1}+\sqrt{c_{1}^{2}-4 c_{2}+8}\right]\right) \frac{1}{\Delta t} \\
& \omega_{2}=\cos ^{-1}\left(\frac{1}{4}\left[-c_{1}-\sqrt{c_{1}^{2}-4 c_{2}+8}\right]\right) \frac{1}{\Delta t}
\end{aligned}
$$

7 The above two equations are different from those in [12], which gave erroneous frequencies 8 and were confirmed to be wrong after the authors worked through the procedure in [12].

9 The direct expression for the frequency for the case of $M=1$ (not considered in [12]) is now 10 derived. Starting from (34), by removing all terms and coefficients related to the second 11 excitation, the equations become

$$
\begin{aligned}
& A_{1} \sin \left(\omega_{1} t_{j}+\psi_{1}\right) \times\left(1+c_{1} \cos \left(\omega_{1} \Delta t\right)+c_{2} \cos \left(\omega_{1} 2 \Delta t\right)\right)=0 \\
& A_{1} \cos \left(\omega_{1} t_{j}+\psi_{1}\right) \times\left(c_{1} \sin \left(\omega_{1} \Delta t\right)+c_{2} \sin \left(\omega_{1} 2 \Delta t\right)\right)=0
\end{aligned}
$$


1 By solving the two equations in (38), the following new expressions for AR coefficients are

2 obtained

$$
\begin{aligned}
& c_{1}=-2 \cos \left(\omega_{1} \Delta t\right) \\
& c_{2}=1
\end{aligned}
$$

3 Hence, for $M=1$, the system described by Eq. (33) becomes:

$$
\left[\begin{array}{c}
{ }^{1} d_{3}+{ }^{1} d_{1} \\
{ }^{1} d_{4}+{ }^{1} d_{2} \\
\vdots \\
{ }^{N_{\text {revs }}} d_{3}+{ }^{N_{\text {revs }}} d_{1} \\
{ }^{N_{\text {revs }}} d_{4}+{ }^{N_{\text {revs }}} d_{2}
\end{array}\right]=\left[\begin{array}{cc}
-\left({ }^{1} d_{2}\right) & 1 \\
-\left({ }^{1} d_{3}\right) & 1 \\
\vdots & \vdots \\
-\left({ }^{N_{\text {revs }}} d_{2}\right) & 1 \\
-\left({ }^{N_{\text {revs }}} d_{3}\right) & 1
\end{array}\right]\left[\begin{array}{l}
C_{1} \\
c_{0}
\end{array}\right]
$$

5 Thus the minimum number of probes in Eq. (40) becomes 4 instead of 5 (for the unreduced 6 case of Eq. (32). Also, the frequency can be calculated as

$$
\omega_{1}=\left[\cos ^{-1}\left(-c_{1} / 2\right)\right] / \Delta t
$$

7 Once the vibration frequencies are known, the residual DC offset and amplitudes/phases in 8 Eq. (25) can be determined from the matrix format of the alternative expression of Eq. (23) 9 for different probes $j=1 \ldots N_{\mathrm{Pr}}$ for any arbitrarily chosen revolution:

$$
\left[\begin{array}{cccccc}
1 & \sin \left(\omega_{1} t_{1}\right) & \cos \left(\omega_{1} t_{1}\right) & \cdots & \sin \left(\omega_{M} t_{1}\right) & \cos \left(\omega_{M} t_{1}\right) \\
1 & \sin \left(\omega_{1} t_{2}\right) & \cos \left(\omega_{1} t_{2}\right) & \cdots & \sin \left(\omega_{M} t_{2}\right) & \cos \left(\omega_{M} t_{2}\right) \\
1 & \vdots & \vdots & \vdots & \vdots & \vdots \\
1 & \sin \left(\omega_{1} t_{N_{\mathrm{Pr}}}\right) & \cos \left(\omega_{1} t_{N_{\mathrm{Pr}}}\right) & \cdots & \sin \left(\omega_{M} t_{N_{\mathrm{Pr}}}\right) & \cos \left(\omega_{M} t_{N_{\mathrm{Pr}}}\right)
\end{array}\right] \times\left[\begin{array}{c}
a_{0} \\
a_{11} \\
a_{21} \\
\vdots \\
a_{1 M} \\
a_{2 M}
\end{array}\right]=\left[\begin{array}{c}
d_{1} \\
d_{2} \\
\vdots \\
d_{N_{\mathrm{Pr}}}
\end{array}\right]
$$

\subsection{Multi-frequency sine fitting with data preparation (MSFP)}

11 According to Russhard's method [1], for the case when the data is prepared so that all EOs in 12 Eq. (24) are integers, these EOs are estimated from FE data (Campbell diagram) of the 
1 vibrating system. Using samples from $N_{\text {Pr }}$ probes for a given revolution, Eq. (24) can be

2 written in a matrix form:

$$
\begin{aligned}
{\left[\begin{array}{c}
d_{1} \\
d_{2} \\
\vdots \\
d_{N_{\mathrm{Pr}}}
\end{array}\right]=\left[\begin{array}{cccccc}
1 & \sin \left(\mathrm{EO}_{1} \theta_{1}\right) & \cos \left(\mathrm{EO}_{1} \theta_{1}\right) & \cdots & \sin \left(\mathrm{EO}_{\mathrm{M}} \theta_{1}\right) & \cos \left(\mathrm{EO}_{\mathrm{M}} \theta_{1}\right) \\
1 & \sin \left(\mathrm{EO}_{1} \theta_{2}\right) & \cos \left(\mathrm{EO}_{1} \theta_{2}\right) & \cdots & \sin \left(\mathrm{EO}_{\mathrm{M}} \theta_{2}\right) & \cos \left(\mathrm{EO}_{\mathrm{M}} \theta_{2}\right) \\
\vdots & \vdots & \vdots & \cdots & \vdots & \vdots \\
1 & \sin \left(\mathrm{EO}_{1} \theta_{N_{\mathrm{Pr}}}\right) & \cos \left(\mathrm{EO}_{1} \theta_{N_{\mathrm{Pr}}}\right) & \cdots & \sin \left(\mathrm{EO}_{\mathrm{M}} \theta_{N_{\mathrm{Pr}}}\right) & \cos \left(\mathrm{EO}_{\mathrm{M}} \theta_{N_{\mathrm{Pr}}}\right)
\end{array}\right]\left[\begin{array}{c}
a_{0} \\
a_{11} \\
a_{21} \\
\vdots \\
a_{1 M} \\
a_{2 M}
\end{array}\right] } \\
\text { Or } \mathbf{d}=\mathbf{B a}
\end{aligned}
$$

3 Unlike MGARES, the probes need not to be equally spaced and the probe angular positions

4 can be optimised to minimise sensitivity of the solution to the probes positions. This is done

5 by calculating the condition number of the matrix $\mathbf{B}$ i.e. the ratio of the biggest to smallest

6 singular values of $\mathbf{B}$. The solution is considered sensitive to small changes in $\theta_{j}$ values if the

7 condition number was greater than ten [1]. A number of iterations should be done using

8 initial positions of the probes until an acceptable solution for the condition number found.

9 Eq. (44) is solved for each individual revolution using Gaussian elimination or Matlab 10 operation mldivide [31] in order to obtain the sine and cosine coefficients $a_{1 m}, a_{2 m}$ for each

$11 \quad \mathrm{EO}_{m}$ over the revolution considered.

12 The last step in the identification for integer EO amplitudes and phases is the validity of the estimates for the EOs used in the fit. This is done using the Pearson correlation factor given in Eq. (45), where $d_{j}$ are the elements of the measurements vector $\mathbf{d}$ on the left hand of eq. (44) and $\hat{d}_{j}$ are the elements of the vector of the fitted measurements $\hat{\mathbf{d}}=\mathbf{B a}$ is as calculated from the right hand of eq. (44) after finding a. The correlation is considered acceptable if it is close to the experimentally validated value of 0.95 [1]. 


$$
\rho_{d_{j} \widehat{d}_{j}}=\frac{\sum_{j=1}^{N_{\operatorname{Pr}}} d_{j} \widehat{d}_{j}-\frac{\left(\sum_{j=1}^{N_{\operatorname{Pr}}} d_{j}\right)\left(\sum_{j=1}^{N_{\mathrm{Pr}}} \widehat{d}_{j}\right)}{N_{\operatorname{Pr}}}}{\sqrt{\left(\left(\sum_{j=1}^{N_{\mathrm{Pr}}} d_{j}^{2}\right)-\frac{\left(\sum_{j=1}^{N_{\mathrm{Pr}}} d_{j}\right)^{2}}{N_{\mathrm{Pr}}}\right)\left(\left(\sum_{j=1}^{N_{\mathrm{Pr}}} \widehat{d}_{j}^{2}\right)-\frac{\left(\sum_{j=1}^{\left.N_{\mathrm{Pr}} \widehat{d}_{j}\right)^{2}}\right.}{N_{\mathrm{Pr}}}\right)}}
$$

1 Although the number of unknowns in Eq. (44) is $2 M+1$, an equal number of probes would 2 result in $\rho_{d_{j} \widehat{d}_{j}}$ being identically 1 , which is not useful. Hence, the minimum number of probes 3 with this method is one more than this, that is

$$
N_{\operatorname{Pr}_{\text {min }}}=2 M+2
$$

4 If the correlation between $d_{j}$ and $\hat{d}_{j}$ is lower than expected, then from the Campbell diagram 5 one should be able to identify an additional EO that was missed out from eq. (43). Access to 6 the Campbell diagram ensures that fake EOs are not added to improve the correlation. In 7 addition to this, there are other precautions that can be taken e.g.

a. Since Eq. (43) is solved for each revolution, the evolution of the phase for each EO response can be examined to confirm resonance.

b. The correlation between EO response amplitudes in a fit can be calculated - for an incorrect EO fitting, a high correlation is registered [1].

c. Responses that are too noisy can simply be ignored - in real applications this is more acceptable than spending high amount of cost and time analysing a response that presents no danger to the blade life.

For the case when the data is prepared so that all EOs in Eq. (24) are non-integers, the solution process for the amplitudes and phases follows that of Eq. (44), except that the EOs used in the matrix are identified using the travelling wave method as follows. 
1 For blisk vibration at circular frequency $\omega_{m}$, the data from all the blades observed by a given

2 probe defines travelling wave of frequency $f_{\mathrm{t}, m} \mathrm{~Hz}[32]$ :

$$
f_{\mathrm{t}, m}=\left(\omega_{m}+N_{\mathrm{ND}_{m}} \Omega\right) /(2 \pi)
$$

3 where $N_{\mathrm{ND}_{m}}$ is the number of nodal diameters of the blisk vibration at $\omega_{m} \cdot N_{\mathrm{ND}_{m}}$ could be 4 determined from the phase difference $\left(\Delta \psi_{m}\right)$ between the travelling waves observed by two 5 probes 1, 2 with angular spacing $\Delta \theta[33]$ as follows:

$$
N_{\mathrm{ND}_{m}}=\Delta \psi_{m} / \Delta \theta
$$

6 Let ${ }_{k}^{n} d_{j}$ denote the displacements observed by a given probe no. $j$ over any given revolution $7 n$, where $k=1 \ldots N_{\mathrm{b}}$ is the blade counter. Over that revolution, the probe observes the time 8 series $\left\{{ }_{1}^{n} d_{j}, \ldots,{ }_{N_{\mathrm{b}}}^{n} d_{j}\right\}$. Let ${ }^{n} X_{j}(f)$ be the Fast Fourier Transform (FFT) of the time series:

$$
{ }^{n} X_{j}(f)=\operatorname{FFT}\left(\left\{\begin{array}{l}
n \\
1
\end{array} d_{j}, \quad \cdots \quad,{ }_{N_{\mathrm{b}}}^{n} d_{j}\right\}\right)
$$

$9 \omega_{m}$ (and therefore $\mathrm{EO}_{m}=\omega_{m} / \Omega$ ) can be determined from Eq. (47) after $f_{\mathrm{t}, m}$ and $N_{\mathrm{ND}_{m}}$ have 10 been determined using the travelling wave frequency spectra ${ }^{n} X_{1}(f),{ }^{n} X_{2}(f)$ at two probes nos. 1, 2, as per following process, which elucidates that described in [1].

12 1- Calculate ${ }^{n} X_{1}(f)$ and ${ }^{n} X_{2}(f)$ for each revolution $n$ of a large number of revolutions while the speed is increasing (it is assumed constant for each revolution).

2- Plot the variation of ${ }^{n} X_{1}(f)$ or ${ }^{n} X_{2}(f)$ with the revolution number $n$ (or rotational speed) to produce a "travelling wave plot". This is illustrated in Fig. 7(a,b) which refers to Test no. 2 in Table 2 of section 5. The variation of $f_{\mathrm{t}, m}$ with rotational speed is clearly identifiable (see Fig. 7(b)). 
13 - From the travelling wave plot, detect a value of $f_{\mathrm{t}, m}$ for which the amplitude is suitably high and record the corresponding revolution number $n=n_{\mathrm{S}}$ and speed $\Omega=\Omega_{n_{\mathrm{S}}}$.

3 4- For the selected speed $\Omega_{n_{\mathrm{S}}}$, calculate the cross spectrum using the following relation [34]

$$
{ }^{n_{\mathrm{S}}} X_{12}(f)={ }^{n_{\mathrm{S}}} X_{1}(f) \cdot{ }^{n_{\mathrm{S}}} X_{2}^{*}(f)
$$

4 where ${ }^{n_{\mathrm{S}}} X_{2}^{*}(f)$ is the complex conjugate of ${ }^{n_{\mathrm{S}}} X_{2}(f)$.

5 5- Calculate $\Delta \psi$ as follows

$$
\Delta \psi=\tan ^{-1}\left[\frac{\operatorname{Im}\left\{{ }^{n_{\mathrm{s}}} X_{12}\left(f=f_{\mathrm{t}, m}\right)\right\}}{\operatorname{Re}\left\{{ }^{n_{\mathrm{s}}} X_{12}\left(f=f_{\mathrm{t}, m}\right)\right\}}\right]
$$

6 6- Calculate $N_{\mathrm{ND}_{m}}$ from equation (48).

7 7- Calculate $\omega_{m}$ from equation (47), and then determine the non-integral engine order $8 \quad \mathrm{EO}_{m}=\omega_{m} / \Omega_{n_{\mathrm{S}}}$.

\section{Fig.7 HERE}

\subsection{Two Parameter Plot Method}

2PP requires data from two probes only and is applicable for synchronous vibration at single frequency $\omega=\mathrm{EO} \Omega$ where EO is a fixed integer and $\Omega$ varies gradually. From Eq. (24), neglecting the DC offset, the tip displacements $d_{1}, d_{2}$ observed at two probes positioned at angles $\theta_{1}, \theta_{2}$ relative to the positive $x$-axis where $\theta_{2}>\theta_{1}$ for $\Omega$ anticlockwise, can be written as:

$$
d_{1}(\omega)=A(\omega) \sin \left(\mathrm{EO} \theta_{1}+\psi(\omega)\right), \quad d_{2}(\omega)=A(\omega) \sin \left(\operatorname{EO} \theta_{1}+\operatorname{PSR}+\psi(\omega)\right)
$$


$\operatorname{PSR}=\operatorname{EO}\left(\theta_{2}-\theta_{1}\right)$

1 The assumed expressions are valid when the blisk vibration is predominantly in a single

2 mode [9]. Hence, as $\Omega$ (and therefore $\omega$ ) varies, the amplitude and phase will vary according

3 to the frequency response function of a single-degree-of-freedom system:

$A(\omega)=\frac{F_{r}}{K_{r}}\left|\frac{1}{1-\left(\omega / \varpi_{r}\right)^{2}+\mathrm{j} 2 \zeta_{r}\left(\omega / \varpi_{r}\right)}\right|, \quad \psi(\omega)=\arg \left\{\frac{1}{1-\left(\omega / \varpi_{r}\right)^{2}+\mathrm{j} 2 \zeta_{r}\left(\omega / \varpi_{r}\right)}\right\}$

4 where $\varpi_{r}, \zeta_{r}, K_{r}$ are the natural circular frequency, damping ratio and equivalent modal

5 stiffness of mode no. $r$ and $\omega, F_{r}$ are the circular frequency and fixed amplitude of the

6 equivalent modal excitation force $f_{r}(t)=F_{r} \sin \omega t$.

7 It is observed that when sweeping $\Omega$ either up or down to traverse the resonance frequency,

8 the plot of $d_{2}(\omega)$ versus $d_{1}(\omega)$ forms an ellipse inscribed in a square of sides of length equal

9 to the amplitude of vibration at resonance $A\left(\varpi_{r}\right)$ i.e. $A\left(\varpi_{r}\right)$ is equal to the peak-to-peak

variation in $d_{1,2}(\omega)$. Moreover, the major axis of the ellipse is always at an angle $\beta$ of $\pm 45^{\circ}$

to the $d_{1}$ axis, except for the PSR equal to an odd multiple of $\pi / 2$, when it degenerates into a circle. This is illustrated in Fig. 8 for the case EO $=1, \theta_{1}=30^{\circ}, \theta_{2}=315^{\circ}$ where Eqs. (51-

53) were used with $\zeta_{r}=0.001$ to compute the non-dimensional displacements

$\tilde{d}_{1,2}(\omega)=d_{1,2}(\omega) /\left(F_{r} / K_{r}\right)$

14 The ellipse in Fig. 8 is inscribed in a square with side of length $\tilde{A}\left(\varpi_{r}\right)=A\left(\varpi_{r}\right) /\left(F_{r} / K_{r}\right)$. It 15 is noted that the ellipse and square were shifted so that their centres coincide with the origin.

16 This was achieved by plotting $\left[\tilde{d}_{2}(\omega)-\overline{\tilde{d}_{2}(\omega)}\right]$ versus $\left[\tilde{d}_{1}(\omega)-\overline{\tilde{d}_{1}(\omega)}\right]$ where $\overline{\tilde{d}_{1,2}(\omega)}$ 17 denotes the mean of the points $\tilde{d}_{1,2}(\omega)$ over the ellipse. 
1 By plotting a number of ellipses for different PSR, Heath [9] identified the following

2 relationships between PSR and the ratio $\varepsilon$ of the length of the minor axis to the length of

3 major axis (Fig. 8), according to the major axis inclination $\beta$ :

$$
\begin{aligned}
& \beta=+45^{\circ}: \quad \text { PSR }=\left\{\begin{array}{c}
-7.22644 \varepsilon^{3}-21.82787 \varepsilon^{2}+119.08946 \varepsilon-0.16976 \\
\text { or } \\
7.21876 \varepsilon^{3}+21.84879 \varepsilon^{2}-119.10273 \varepsilon+360.17127
\end{array}\right. \\
& \beta=-45^{\circ}: \quad \operatorname{PSR}=\left\{\begin{array}{c}
7.27262 \varepsilon^{3}+21.84211 \varepsilon^{2}-119.16418 \varepsilon+180.17559 \\
\text { or } \\
-7.28363 \varepsilon^{3}-21.82579 \varepsilon^{2}+119.15622 \varepsilon+179.82559
\end{array}\right.
\end{aligned}
$$

4 Hence, from an experimental plot $\left[d_{2}(\omega)-\overline{d_{2}(\omega)}\right]$ versus $\left[d_{1}(\omega)-\overline{d_{1}(\omega)}\right]$ one can extract

5 two different values of PSR, and knowing the probe relative spacing $\theta_{2}-\theta_{1}$, two different

6 values of EO can be calculated from Eq. (52). From these, the one that most closely

7 corresponds to an integer should be selected. In case that both EO estimates closely 8 correspond to integers, a prior knowledge of EO would be essential. A two parameter plot of data generated by the simulator of this paper and corrupted by 5\% NSR is shown in Fig. 9. Rigosi et al [15] recently (2016) claimed improvements to the $2 \mathrm{PP}$ method through an analytical method of estimating the PSR. Their analysis appears to assume that $d_{1}(\omega)=$ $A(\omega)$ at a point where the ellipse touches one of the vertical sides of the inscribing square. However, the authors of the present paper have found that this may not always be the case.

Fig. 8 HERE

Fig. 9 HERE

\section{VALIDATION OF THE FE MODAL MODEL USED IN STUDY}

The blisk considered in this study was provided by an industrial collaborator and its design is shown in Fig. 10. It was made from an aluminium alloy (rr58) of Young's modulus $72 \mathrm{GPa}$ and density of $2750 \mathrm{~kg} / \mathrm{m}^{3}$. 
3 The blisk was analysed by FE analysis and experimentally in two non-rotating 4 configurations:

5 (1) Free conditions - these conditions were approximately realized experimentally by placing

6 the blisk horizontally on a soft foam support;

7 (2) Centrally mounted conditions i.e. attached to a steel block, as shown in Fig. 2 and the experimental set up in Fig. 11 (where the block is fixed to an optical table).

Fig. 11. HERE

12 Configuration (1) was used as a basic test for the modelling of the blisk in isolation.

Configuration (2) was the one whose modal parameters were used in the simulator for 14 generating the BTT data in section 5 (as per Eqs. (5), (9)). Both configurations were 15 experimentally tested for frequency response functions (FRFs) using impact testing 16 (instrumented hammer and small accelerometers). Configuration (2) was additionally experimentally analysed using two FireWire cameras under electromagnetic shaker excitation for the mode shapes (see Fig. 12).

21 The geometries of both the blisk and (in the case of configuration (2)) the steel block support were built using SolidWorks 2014 and then exported to ANSYS 15.0 for FE analysis. The element used in the mesh was Solid187 (10-node element). In the case of configuration (2), 
1 bottom base of the support block (Fig. 2) was fully fixed and the mating parts of the

2 connection between the blisk and the steel block were meshed using 3-D contact surface

3 element CONTA174 associated with the 3-D target segment elements TARGE170. The

4 numbers of nodes and elements were respectively as follows: 77232, 48215 (conf. (1) - free

5 blisk); 638623, 394592 (conf. (2) - centrally mounted blisk).

6 With regard to conf. (1), the experimental FRFs were in very good agreement with the 7 predicted ones, as illustrated in Fig. 13, where both force and response were normal to the 8 plane of the blisk. The low frequency discrepancy is simply due to the bounce mode of the 9 blisk on the foam support (which was not considered in the prediction made with no constraints). As expected, for low frequencies and ignoring the bounce mode, both measured and predicted accelerance FRFs asymptote towards the reciprocal of the mass of the blisk. There appears to be an unexplained divergence between measurement and prediction in the region 1.9-2 kHz, but nonetheless the overall agreement validates the blisk's FE parameters.

Fig. 14 shows the predicted and measured accelerance FRFs for conf. (2), where both force and response are normal to the plane of the blisk. The agreement is reasonably good, although not as good as for conf. (1). This was expected due to the added complications of the constraints in the model, and the assumption fully fixed conditions at the base of the support structure (Fig. 2) which is difficult to realise experimentally.

With regards to the values of the natural frequencies, there is fair correlation between predictions and measurements, as seen in Table 1, with discrepancies in the first six modes ranging from $0.7 \%$ to $8.1 \%$. It is noticed that only three of these modes appear clearly in the FRF magnitude graph of Fig. 14. 
3 Table 1 Natural frequencies of blisk in configuration (2) (Figs. 2, 11)

\begin{tabular}{ccccccc}
\hline Mode \# & 1 & 2 & 3 & 4 & 5 & 6 \\
\hline Type & Torsional & Bending & Bending & Axial & Bending & Bending \\
Experimental $(\mathrm{Hz})$ & 222 & 370 & 385.9 & 942.5 & 1499.8 & 1520.1 \\
FE $(\mathrm{Hz})$ & 218.7 & 402.6 & 404.7 & 893.7 & 1509.7 & 1509.9 \\
$\%$ Error & 1.8 & 8.8 & 4.9 & -5.2 & 0.7 & -0.7 \\
\hline
\end{tabular}

8 Fig. 15 shows the predicted mode shapes of the first six modes. Mode no. 1 (predicted at $9 \quad 218.7 \mathrm{~Hz}$ ) is a torsional mode which therefore does not feature in the FRFs of Fig. 14 (since the excitation was normal to the blisk). The experimental value of $222 \mathrm{~Hz}$ in Table 1 was therefore determined separately using FireWire cameras/shaker setup (Fig. 12) with tangential excitation. With reference to the FE predicted frequency values in Table 1 and the corresponding mode shapes in Fig. 15:

- Mode nos. 2 and 3 are closely spaced bending modes (402.6, 404.7 Hz) each with a single nodal diameter $\left(N_{\mathrm{ND}}=1\right)$, the respective nodal diameters being orthogonal to each other. These two modes feature as a double peak in the predicted FRF in Fig. 14.

- Mode no. $4(893.7 \mathrm{~Hz})$, is an axial or "umbrella" mode with no nodal diameter.

- Mode nos. 5 and 6 are very closely spaced bending modes (1509.7, 1509.9 Hz) each with a two orthogonal nodal diameters $\left(N_{\mathrm{ND}}=2\right)$. These two modes appear as a single peak in the predicted FRF in Fig. 14. 
1 With reference to the corresponding measured frequencies in Table 1

2 - The frequency estimates $(370,385.9 \mathrm{~Hz})$ for modes nos. 2 and $3\left(N_{\mathrm{ND}}=1\right.$ bending modes) are also closely spaced, but their spacing is not as close as the corresponding predictions (see above). It is noticed that mode no. 3 is a minor, barely visible peak in the measured FRF magnitude of Fig. 14 and its frequency value was identified accurately from the FRF phase.

- $\quad$ The frequency estimates $(1499.8,1520.1 \mathrm{~Hz})$ for modes nos. 5 and $6\left(N_{\mathrm{ND}}=2\right.$ bending modes) are more closely spaced than the frequency estimates for the $N_{\mathrm{ND}}=1$ bending modes, and this trend follows the predictions. It is noticed that mode no. 6 features as a prominent peak in the measured FRF magnitude graph of Fig. 14, whereas mode no.5 is a minor, barely visible peak whose frequency value was identified from the FRF phase.

It is noticed that mode no.4 features prominently in both predicted and measured FRFs of Fig. 14, but the resonance peak of the measurement was highly damped. The FE modelling neglected damping.

The predicted and measured shapes of mode nos. 2 to 5 were analysed using the modal assurance criterion (MAC), from which auto MAC and cross MAC matrices were computed as per method in [35]. The auto MAC matrix [35] contains MAC values comparing the mode shapes from a single source, as per Fig. 16(a), which refers to the FE predictions. This matrix presents the expected relationships between the modes. The cross MAC matrix contains MAC values comparing the mode shapes from two different sources, as per Fig. 16(b), which compares measurements with the predictions. It is clear from Fig. 16(a,b) that the cross MAC matrix very closely matches the auto MAC matrix. The MAC values comparing corresponding mode shapes (i.e. along the diagonal) are close to unity (i.e. modes are matching or "parallel"). Any two non-corresponding (off-diagonal) modes have MAC values that are low or close to zero (i.e. are "orthogonal"), except for the MAC values relating mode 
1 no. 5 (bending with $N_{\mathrm{ND}}=2$ ) with either of mode nos. 2 or 3 (bending with $N_{\mathrm{ND}}=1$ ), for

2 which the MAC is in the region 0.50 to 0.65 (meaning that mode no.5 is neither orthogonal nor parallel with mode nos. 2 or 3 ).

\section{Fig. 16. HERE}

Finally, the convergence of the modal transformation used in the blisk dynamic deflection component of the simulator (Eqs (9) to (11)) was tested for the six modes of conf. (2). Eqs. (11) were integrated for harmonic excitation of unit amplitude in the $z$-direction. The amplitudes of the steady-state time domain response in the $x, y$ and $z$ directions (obtained from Eq. (9)) were then compared with the "exact" values from the FRFs (obtained from ANSYS using modal superposition harmonic analysis with 10 modes). As shown in Fig. 17, which refers to the response at a blade tip, the correlation between time and frequency domains is accurate to within $1 \%$.

Fig. 17. HERE

\section{ASSESSMENT OF BLADE TIP TIMING ALGORITHMS}

\subsection{Description of Tests}

Table 2 summarises the conditions for the five tests conducted using data generated from the simulator, in order to assess MSFP, MGARES and 2PP (where applicable). For tests 1 and 2, the rotational speed range and the EO of the single-frequency excitation were chosen so that the first blisk mode was excited, whereas for tests 3-5, the rotational speed range and the EOs of the double-frequency excitation were chosen so that the first and second/third modes (218.7, $400 \mathrm{~Hz}$ ) were excited (see Fig. 15 (a-c) and Fig. 6). To generate the desired 
1 response, it was sufficient to apply single point excitation at a node on the tip of the topmost

2 blade (Fig. 2). For tests 1, 2, this force was in the $x$-direction, whereas for tests 3-5 it had an

3 additional component in the $z$-direction. The damping ratio in Eq. (11) was taken as 0.05 for

4 all modes. Fig. 18(a-c) show, for Tests 3-5 respectively, the tangential vibratory responses at

5 the nominal sensing point of a given blade tip. This response is obtained from the $x, y$

6 vibrations contained in the vector $\Delta \mathbf{u}^{(\text {def) }}(t)$ (eq. (9)). For a point $\mathrm{P}_{k}$ at an angular position

$7 \quad \theta_{\mathrm{P}_{k}}^{(\mathrm{ref})}$ in the fixed reference orientation, the tangential vibratory response is calculated as

$8 \quad \Delta y_{P_{k}}^{(\mathrm{def})}(t) \cos \theta_{\mathrm{P}_{k}}^{(\mathrm{ref})}-\Delta x_{P_{k}}^{(\mathrm{def})}(t) \sin \theta_{\mathrm{P}_{k}}^{(\mathrm{ref})}$, where $\Delta x_{P_{k}}^{(\mathrm{def})}(t)$ and $\Delta y_{P_{k}}^{(\mathrm{def})}(t)$ are the

9 Cartesian components of the vibratory response at $\mathrm{P}_{k}$ (contained in $\Delta \mathbf{u}^{(\mathrm{def})}(t)$ ). This latter

10 vector is transformed by the simulator into BTT data $d_{j}$, as per Fig. 3.

Fig. 18. HERE

Table 2. Summary of BTT test conditions

\begin{tabular}{cccccc}
\hline Tests & $\begin{array}{c}\text { No. of } \\
\text { synchronous } \\
\text { excitations }\end{array}$ & $\begin{array}{c}\text { No. of } \\
\text { asynchronous } \\
\text { excitations }\end{array}$ & EO & $\begin{array}{c}\text { rotational speed } \\
\text { range (rev/sec) }\end{array}$ & $\begin{array}{c}\text { angular } \\
\text { velocity sweep } \\
\text { duration } T_{\mathrm{s}}(\mathrm{s})\end{array}$ \\
\hline 1 & 1 & 0 & 1 & $150: 250$ & 1 \\
2 & 0 & 1 & 1.5 & $100: 200$ & 1 \\
3 & 2 & 0 & 5,9 & $30: 50$ & 5 \\
4 & 0 & 2 & $1.5,2.7$ & $100: 200$ & 1 \\
5 & 1 & 1 & $2,3.6$ & $75: 125$ & 2 \\
\hline
\end{tabular}

14 Table 3 summarises the probe angles used for the tests. In all cases the minimum required number of probes was used, except for test 5. From Eq. (46), by the MSFP method, $N_{\operatorname{Pr}_{\min }}=$

164 for tests $1,2(M=1$ in Eq. $(24))$ and $N_{\operatorname{Pr}_{\min }}=6$ for tests $3-4(M=2)$. In the case of MGARES, the minimum numbers of probes required by Eq. (40) (single-frequency vibration) and Eq. (36) (two-frequency vibration) were reduced from 5 and 7 respectively to 4 and 6 respectively since the dc offset term $c_{0}$ was taken to be zero as a result of the data preparation 
1 process (in fact, no dc offset was applied in Eq. (5) when generating the raw data). For test 5,

2 a minimum of 4 probes could be used since the data preparation of section 3.1 resulted in two

3 separate single-frequency components - one synchronous, the other non-synchronous.

4 Nonetheless, 6 probes were used for test 5 in order to investigate the effect on MGARES of

5 not separating the integer and non-integer EOs (possible with MGARES but not MSFP).

6 As regards the spacing $\Delta \theta$ of the probe angular positions used in each test (Table 3), this was

7 chosen to produce an acceptable condition number of matrix B used in MSFP (Eq. (44)), and

8 also be within the limits of 30-70\% PSR (see Eq. (3)) recommended for MGARES [12]. Fig.

919 shows the allowable range of values $\Delta \theta$ for test 1 based on the condition of the MSFP 10 matrix $\mathbf{B}$. In the case of tests $3-5$, it was not possible to find a single value of $\Delta \theta$ that 11 satisfied both MSFP and MGARES limits and therefore different values of $\Delta \theta$ were used for MSFP and MGARES.

Table 3. Probe angular positions for each test.

\begin{tabular}{|c|c|c|c|c|c|c|c|}
\hline \multirow{2}{*}{$\frac{\text { Test }}{1}$} & & \multicolumn{6}{|c|}{$\begin{array}{c}\text { Probe angles (degrees) } \\
\text { (measured from positive } x \text {-axis, anti-clockwise positive) }\end{array}$} \\
\hline & & -120 & -59 & 2 & 63 & - & - \\
\hline 2 & & -100 & -59 & -18 & 23 & - & - \\
\hline \multirow{2}{*}{3} & MSFP & 0 & 13 & 26 & 39 & 52 & 65 \\
\hline & MGARES & 0 & 5 & 10 & 15 & 20 & 25 \\
\hline \multirow{2}{*}{4} & MSFP & -140 & -97 & -54 & -11 & 32 & 75 \\
\hline & MGARES & -10 & 7 & 24 & 41 & 58 & 75 \\
\hline \multirow{2}{*}{5} & MSFP & -100 & -68 & -36 & -4 & 28 & 60 \\
\hline & MGARES & 0 & 13 & 26 & 39 & 52 & 65 \\
\hline
\end{tabular}


2 Figs. 20-24 show, for each test respectively, the tip displacement data $d_{j}$ for a given blade at each probe, as generated by the simulator for force amplitudes of $1 \mathrm{~N}$, prior to the addition of noise and data preparation (section 3.1). In the case of MSFP with non-integral EOs, the data for all blades at two probes were additionally required, as per travelling wave method (section 3.3). The BTT data profiles for the tests that have only integer EOs (Figs. 20 and 22) are seen to be smooth, whereas presence of non-integer EOs results in spiky BTT data profiles (Figs. 21, 23, 24). With regard to Fig. 20, which refers to a single integer EO, the peak-to-peak variation in displacement for all probes is the same, agreeing with the basic assumption of the 2PP method [36]. It is noted that the slight ripples at the start of each profile in Figs. 20, 22 are due to the initial transients of the time domain integration process.

Fig. 21. HERE

Fig. 22. HERE

Fig. 23. HERE

\section{Fig. 24. HERE}

The data in Figs. 20-24 were corrupted with various levels of noise (0, 5, 10, $20 \%$ NSR) before data preparation as per section 3.1 and the application of the BTT algorithms for frequency and amplitude estimation. For MGARES, the value of $N_{\text {revs }}=11$ (Eqs. (36), (40)) and the results were checked for repeatability with $N_{\text {revs }}=21$ and again with $N_{\text {revs }}=$ 
13 . The repeatability of the output of the BTT algorithms was also checked for BTT data

2 generated at higher force amplitudes $(10 \mathrm{~N}, 100 \mathrm{~N})$, in view of the reported influence of 3 amplitude on the cross-correlation factor of Eq. (22) [1].

\section{$4 \quad 5.2$ Results and Discussion}

5 The resonant tip vibration response that is recovered from the BTT amplitude, phase and 6 EO/frequency estimates can be described as follows

$$
u=a_{0}+\sum_{m=1}^{M} A_{m} \sin \left(\mathrm{EO}_{m} \Omega_{\mathrm{res}} t+\psi_{m}\right)
$$

7 The above expression brings together all EOs, integer and non-integer, and is compared with

8 the benchmark signal, which is the tangential blade tip vibration generated by the simulator in 9 the region of the resonance speed $\Omega_{\text {res }}$ at which one mode resonates (Tests 1,2 ) or two modes resonate simultaneously (Tests 3-5, Fig. 18). Fig. 25(a-c) compare, for Tests 3-5 respectively, the estimated BTT frequencies and amplitudes with the FFT of a short-duration dataset of the benchmark signal centred around the resonance point $\Omega(t)=\Omega_{\text {res }}$ of the speedsweep response (Fig. 18(a-c)). It is seen that FFT is good for checking the BTT frequencies. However, FFT is not suitable for fairly assessing the BTT amplitudes since the speed is only exactly resonant at the centre of the time history used for the FFT. The BTT amplitudes are therefore assessed by comparing the half peak-to-peak amplitude of the reconstructed signal (Eq. (56)) with that of the frequency sweep response (Fig. 18(a-c) for Tests 3-5) at the exact point where $\Omega(t)=\Omega_{\text {res }}$. Table 4 shows the BTT amplitude errors for all tests. 
Table 4. Percentage errors in BTT half peak-to-peak amplitudes for all tests (the reference signals for tests 3-5 are illustrated in Figs. 18(a-c)).

\begin{tabular}{lcccccc}
\hline Test \# & \multicolumn{2}{c}{ MSFP } & \multicolumn{2}{c}{ MGARES } & \multicolumn{2}{c}{ 2PP } \\
& No noise & Added noise & No noise & added noise & No noise & added noise \\
\hline $\mathbf{1}$ & 0.3 & -.45 & 0.65 & 0.3 & 1.7 & 1.9 \\
$\mathbf{2}$ & -0.7 & 11.8 & 0.08 & 10.2 & & \\
$\mathbf{3}$ & -0.01 & -3.9 & 1.2 & -25.2 & & \\
$\mathbf{4}$ & 3.4 & 11.6 & 4.4 & -10.1 & & \\
$\mathbf{5}$ & -2.8 & -11.8 & -3.3 & 5.6 & & \\
\hline
\end{tabular}

3

4 Table 5 summarises the results of the application of MSFP, MGARES and 2PP to the BTT

5 data after the data preparation process that includes the separation of integer EOs from non-

6 integer EOs. Hence, the double-frequency entries refer to cases where the two EOs are either

7 both integral (test 3, Table 2) or both non-integral (test 4) and the single-frequency entries

8 refer to tests 1,2 and 5. In all cases, the amplitude error is calculated as described above.

9 With regards to frequency error, in case of Tests 3-5, each BTT method is classified

10 according to the higher of the individual errors of the two frequencies.

11 Table 5 quantifies the flexibility of implementation of each method based on the allowable

12 variation in the probe spacing as follows

$\%$ allowable variation in probe spacing $=100 \times \frac{\left(\Delta \theta_{\max }-\Delta \theta_{\min }\right)}{\left(\Delta \theta_{\text {max }}\right)_{\text {phys }}}$

13 where:

14 - $\left(\Delta \theta_{\mathrm{max}}\right)_{\mathrm{phys}}=360^{\circ} / N_{\mathrm{Pr}_{\min }}$ is the maximum probe spacing that is physically possible;

$15-\Delta \theta_{\max }$ and $\Delta \theta_{\min }$ are the maximum and minimum allowable probe spacings.

16 In the case of MSFP, $\Delta \theta_{\max }, \Delta \theta_{\min }$ were calculated as per method in Fig. 19. In the case of

17 MGARES, $\Delta \theta_{\max }, \Delta \theta_{\min }$ were determined from Eq. (3) using the PSR limits (30-70\%) 
1 determined by [12]. For the cases involving MSFP with integer EOs, the Campbell diagram

2 of the blisk was used to identify the frequencies. The present version of the simulator does

3 not consider gyroscopic and centrifugal stress effects and so the Campbell diagram is a plot

4 of the constant FE frequencies in Table 1 versus the rotational speed. In the absence of such

5 information, the synchronous frequencies for the MSFP can be determined from BTT data of

6 all blades using the travelling wave method, as done for the case of asynchronous vibration

7 with MSFP, where the frequency values were identified with negligible error (Table 5).

8 Table 5 shows that, in the absence of noise, MSFP, MGARES and 2PP have negligible error 9 in frequency and negligible/low error in amplitude (where negligible error is $<1 \%$ and low error is $1-5 \%$ ). When noise is applied, all frequency estimates of MSFP remain accurate, as well as those of 2PP (low error), and MGARES single excitation (negligible to low). However, the frequencies of double excitations from MGARES have high (20-50\%)/very high $(>50 \%)$ errors. With regard to amplitude estimation under noise, the error is low for 2PP, negligible for MSFP with synchronous vibration (single/double-frequency), and moderate (5-20\%) for MSFP with asynchronous vibration (single/double-frequency). The amplitude error for MGARES under noise was negligible for synchronous single-frequency, moderate for asynchronous single-frequency, and moderate to high for double-frequency vibration.

The results in Table 5 show that the worst performance under noise was registered by MGARES when applied to double-frequency vibration. For this case, the MGARES also became very rigid with regard to allowable variation in probe spacing. Overall, MSFP allowed greater flexibility in probe spacing and showed best performance overall. $2 \mathrm{PP}$ is not restricted by probe spacing but is limited to single-frequency synchronous vibrations. 
1 It is important to report the effect of the data preparation process of section 3.1 on the results

2 in Table 5. This had most effect on the results of tests 1,3 , and 5 of Table 2 . In the case of

3 test 5 with $20 \%$ NSR, if the integral and non-integral EOs were not separated before applying

4 MGARES, the errors in the integer EO frequency, non-integer EO frequency and non-integer

5 EO amplitude would rise to $52 \%, 139 \%$ and $40 \%$ respectively (from $1.3 \%, 4.3 \%$ and $5.6 \%$

6 respectively). Smoothing significantly improved the estimation of the amplitude/frequency in

7 all cases involving integer EO vibration components e.g. for $2 \mathrm{PP}$, the amplitude and

8 frequency errors respectively reduced from $9.6 \%$ and $10 \%$ to only $1.9 \%$ and $4 \%$ in test 1 at

$920 \%$ NSR; for MGARES, the frequency error reduced from $174 \%$ to $50.2 \%$ in test 3 at $20 \%$

10 NSR. 
Table 5. Performance comparison between BTT algorithms for cases studied (error designations as follows: negligible <1\%; low 1-5\%; moderate 5-20\%; high 20-50\%; very high >50\%).

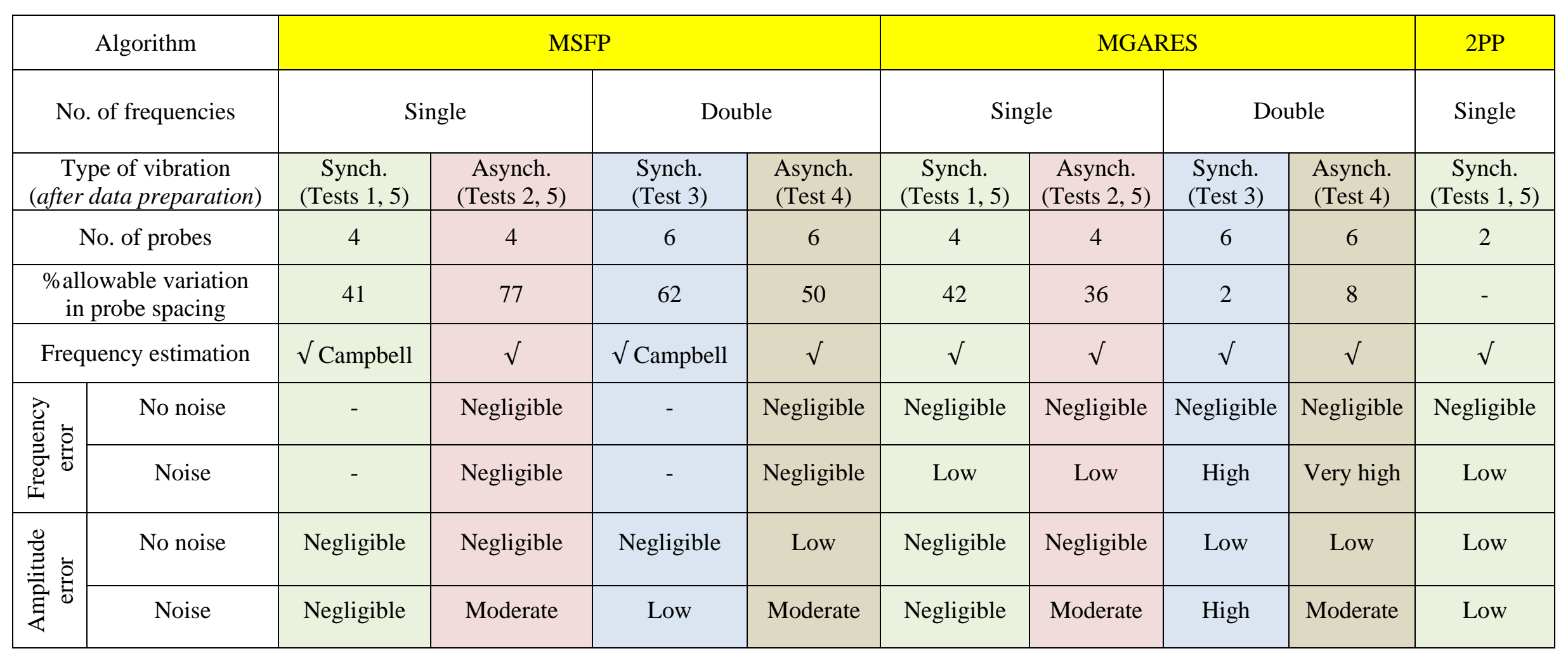




\section{6. CONCLUSIONS}

2 This paper presented a novel simulator based on the FE-derived reduced modal model of a

3 bladed disk (blisk) assembly for the generation of BTT data. The simulator has features and

4 capabilities that are similar to another modal model simulator derived by other researchers at

5 the same time, with the notable difference that the present simulator can output the exact

6 blade passing times, rather than approximate ones based on interpolation. The simulator was

7 used to generate BTT data to assess the performance of three principal BTT algorithms:

8 multi-frequency sine fitting with data preparation (MSFP); multiple global autoregressive

9 (“MGARES”); 2-parameter plot (2PP). New formulae were derived for the identification by

10 MGARES of two-frequency and single-frequency vibrations. The modal model of the blisk

11 used for the assessment was experimentally validated. The BTT data for all methods were prepared in the same way through the minimisation of added AC/DC noise and separation of integer and non-integer EOs. For this study, the worst performance under noise was the case of MGARES applied to two-frequency vibration (both synchronous or both nonsynchronous), which also required tight restrictions on variability in probe spacing. The separation of integer from non-integer EOs in the data preparation process had a significant beneficial effect on MGARES, as did data smoothing for the identification of integer EOs by all three methods. Overall, MSFP registered best overall performance - this was partly due to the fact that the identification procedure for the frequencies (EOs) used in this method was independent of the fitting process. Further assessment of BTT algorithms using a blisk model with relatively long blades will be presented in a future study. 
2 This work was funded by the Egyptian Ministry of Higher Education and the British Council

3 in Cairo. We wish to express our sincere thanks to Rotadata Ltd, Derby, UK, for its support,

4 including access to facilities.

\section{REFERENCES}

6

[1] P. Russhard, Development of a Blade Tip Timing Based Engine Health Monitoring System, The University of Manchester, 2010.

[2] K.K. R. Przysowa, Triggering Methods in Blade Tip-timing Systems, in: Book Vibration Engineering and Technology of Machinery, VETOMAC-XII Ed., Warsaw, Poland, 2016.

[3] M. Mohamed, P. Bonello, P. Russhard, The Determination of Steady-State Movements Using Blade Tip Timing Data, in: Volume 7C: Structures and Dynamics, ASME, Oslo, Norway, 2018.

[4] M. Mohamed, P. Bonello, P. Russhard, A novel method for the determination of the change in blade tip timing probe sensing position due to steady movements, Mechanical Systems and Signal Processing. 126 (2019) 686-710.

[5] M. Mohamed, P. Bonello, P. Russhard, The Determination of Simultaneous SteadyState Movements Using Blade Tip Timing Data, in: Volume 7C: Structures and Dynamics, ASME, Phoenix, Arizona, USA, n.d.: pp. 1-12.

[6] I.B. Carrington, Development of Blade Tip Timing Data Analysis Techniques, University of Manchester, 2002.

[7] A.S. Lee, A.F. Vedichtchev, LP compressor blade vibration characteristics at starting conditions of a $100 \mathrm{MW}$ heavy-duty gas turbine, KSME International Journal. 18 (2004) 895-903.

[8] T.M. Pickering, Methods for Validation of a Turbomachinery Rotor Blade Tip Timing System Methods, Virginia Polytechnic Institute and State University, 2014.

[9] S. Heath, A New Technique for Identifying Synchronous Resonances Using TipTiming, Journal of Engineering for Gas Turbines and Power. 122 (2000) 219-225.

[10] S. Heath, A Study of Tip-Timing Measurement Techniques for the Determination of Bladed-Disk Vibration Characteristics, The University of London, 1996. 
[11] I. B. Carrington, J. R. Wright, J. E. Cooper, G. Dimitriadis, A Comparison of Blade Tip-Timing Data Analysis Methods, Proceedings of the 1st International Conference on the Integration of Dynamics, Monitoring and Control. 215 (1999) 1-6.

[12] J. Gallego-Garrido, G. Dimitriadis, J.R. Wright, A Class of Methods for the Analysis of Blade Tip Timing Data from Bladed Assemblies Undergoing Simultaneous Resonances-Part I: Theoretical Development, International Journal of Rotating Machinery. (2007) 1-11.

[13] J. Gallego-Garrido, G. Dimitriadis, I.B. Carrington, J.R. Wright, A Class of Methods for the Analysis of Blade Tip Timing Data from Bladed Assemblies Undergoing Simultaneous Resonances-Part II: Experimental Validation, International Journal of Rotating Machinery. (2007) 1-10.

[14] Y.-J.Y. and A. von F. Kyu-Kang Joung, Suk-Chul Kang, Ki-Seok Paeng, No-Gill Park, Ho-Jin Choi, Analysis of Vibration of The Turbine Blades Using Non-Intrusive Stress Measurement System, in: ASME 2006 Power Conference, 2006: pp. 1-7.

[15] G. Rigosi, G. Battiato, T.M. Berruti, Synchronous vibration parameters identification by tip timing measurements, Mechanics Research Communications. 79 (2016) 7-14.

[16] P. Händel, Properties of the IEEE-STD-1057 four-parameter sine wave fit algorithm, IEEE Transactions on Instrumentation and Measurement. 49 (2000) 1189-1193.

[17] K. Kaźmierczak, R. Przysowa, Standard sine fitting algorithms applied to Blade Tip Timing data, Journal of KONBiN. 30 (2014) 21-30.

[18] J. Gallego-Garrido, G. Dimitriadis, J.R. Wright, Development of a multiple modes simulator of rotating bladed assemblies for blade tip-timing data analysis, Proceedings of the 2002 International Conference on Noise and Vibration Engineering. (2002) 1437-1446.

[19] G. Dimitriadis, I.B. Carrington, J.R. Wright, J.E. Cooper, Blade-Tip Timing Measurement of Synchronous Vibrations of Rotating Bladed Assemblies, Mechanical Systems and Signal Processing. 16 (2002) 599-622.

[20] U. Pfeifer, M. Zidorn, Tip timing measurement chain validation with the Universal Tip Timing Calibrator UTTC, approach and experience, in: AIP Conference Proceedings, 2012: pp. 43-51.

[21] L. Piechowski, R. Rzadkowsk, P. Troka, P. Piechowski, L. Kubitz, R. Szczepanik, TipTiming Steam Turbine Rotor Blade Simulator, Journal of Vibration Engineering \& Technologies. 6 (2018) 317-323.

[22] V. Kharyton, J. Laine, F. Thouverez, O. Kucher, Simulation of Tip-Timing Measurements of a Cracked Bladed Disk Forced Response, Proceedings of ASME Turbo Expo. (2010) 1-10.

[23] D.H. Diamond, P.S. Heyns, A.J. Oberholster, A Comparison Between Three Blade Tip Timing Algorithms for Estimating Synchronous Turbomachine Blade Vibration, in: 
Proceedings of 2014 World Congress on Engineering Asset Management, 2015: pp. $215-225$.

[24] F. Figaschewsky, B. Hanschke, A. Kühhorn, Efficient Generation of Engine Representative Tip Timing Data Based on a Reduced Order Model for Bladed Rotors, Proceedings of ASME Turbo Expo. (2018) 1-12.

[25] O. Jousselin, Development of Blade Tip Timing Techniques in Turbo Machinery, The University of Manchester, 2013.

[26] J. Strnad, J. Liska, Diagnostic methods of a bladed disc mode shape evaluation used for shrouded blades in steam turbines, Journal of Physics: Conference Series. 659 (2015).

[27] G.L. Forbes, R.B. Randall, Estimation of turbine blade natural frequencies from casing pressure and vibration measurements, Mechanical Systems and Signal Processing. 36 (2013) 549-561.

[28] Ansys, Theory Reference for the Mechanical APDL and Mechanical Applications, 2009.

[29] A. Savitzky, M.J.E. Golay, Smoothing and Differentiation of Data by Simplified Least Squares Procedures, Analytical Chemistry. 36 (1964) 1627-1639.

[30] C. Mathworks, Image Processing Toolbox ${ }^{\mathrm{TM}}$ Reference R 2018 a, (2018).

[31] R. Przysowa, Analysis of synchronous blade vibration with the use of linear sine fitting, Journal of KONBiN. 2 (2014) 17.

[32] I. García, J. Beloki, J. Zubia, G. Aldabaldetreku, M. Illarramendi, F. Jiménez, An Optical Fiber Bundle Sensor for Tip Clearance and Tip Timing Measurements in a Turbine Rig, Sensors. 13 (2013) 7385-7398.

[33] W.B. Watkins, R. a Y.M. Chi, Noninterference blade-vibration measurement system for gas turbine engines, Journal of Propulsion and Power. 5 (1989) 727-730.

[34] W. Watkins, W. Robinson, R. Chi, Noncontact engine blade vibration measurements and analysis, in: 21st Joint Propulsion Conference, American Institute of Aeronautics and Astronautics, Reston, Virigina, 1985.

[35] M. Pastor, M. Binda, T. Harčarik, Modal assurance criterion, Procedia Engineering. 48 (2012) 543-548.

[36] S. Heath, M. Imregun, An Improved Single-Parameter Tip-Timing Method For Turbomachinery Blade Vibration Measurement Using Optical Laser Probes, International Journal of Mechanical Sciences. 38 (1996) 1047-1058. 


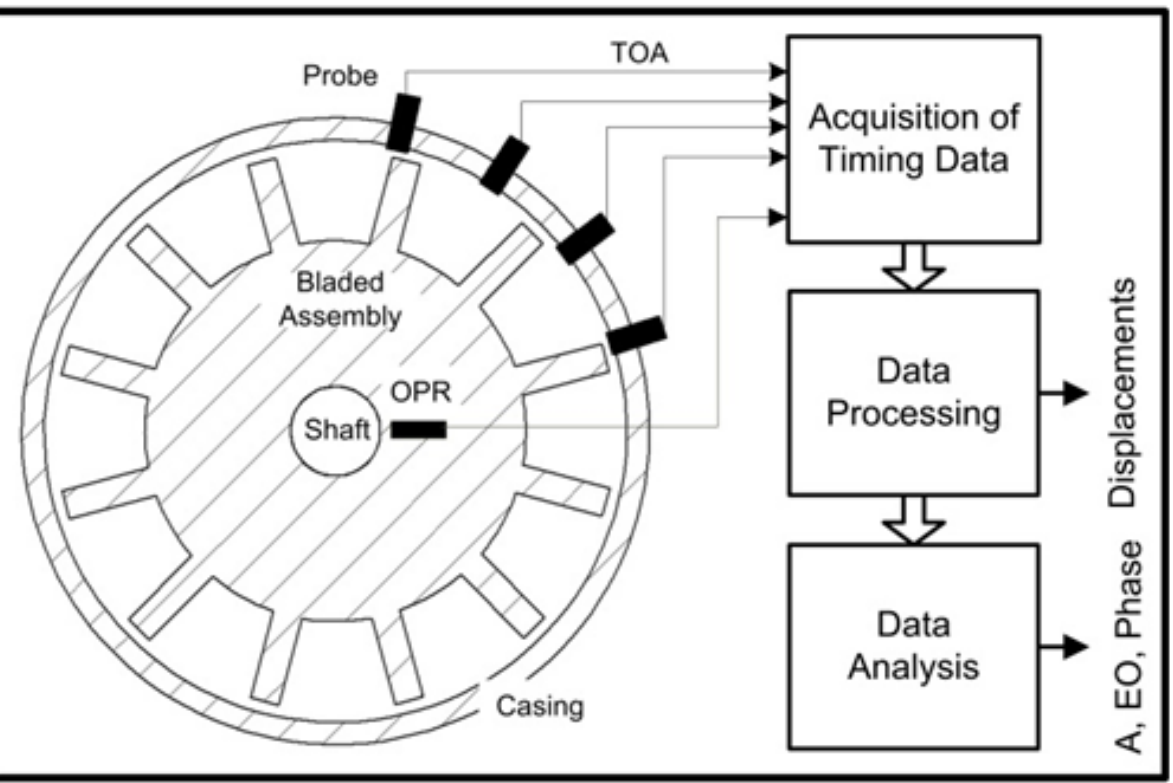

Fig. 1. BTT data flow

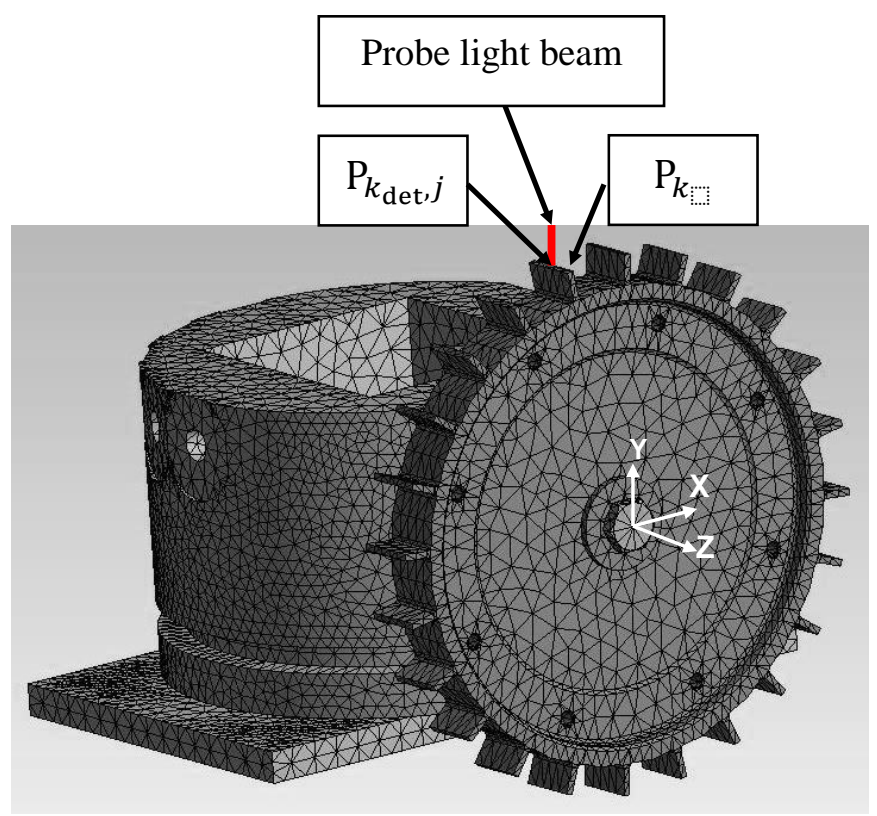

Fig. 2. Instantaneous position of a blade tip node 


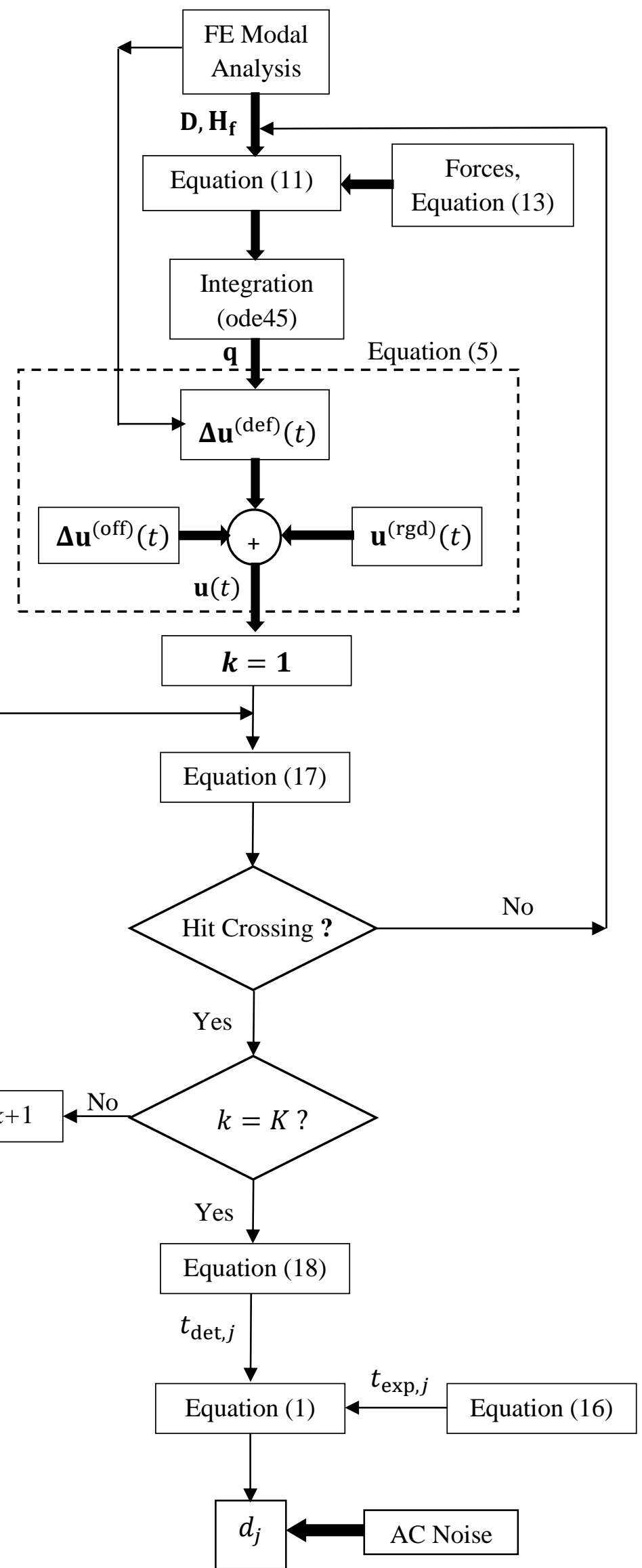

Fig. 3. Flow chart of the simulator 
(a) $3 \times 10^{-5}$

- Raw data

3

4

5

6

7

8

9 
(a) $3 \times 10^{-5}$

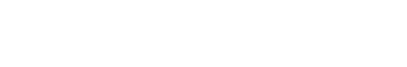

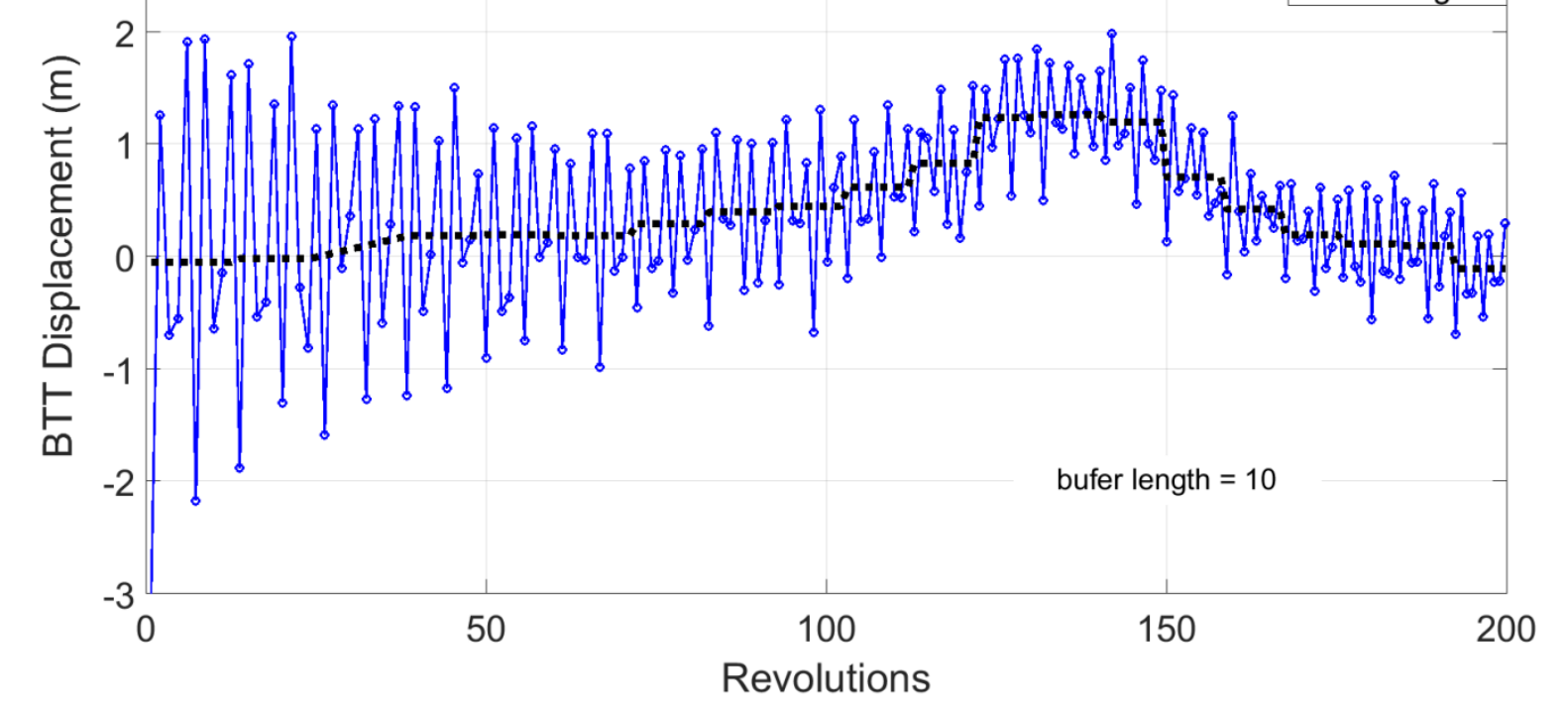

(b) $3 \times 10^{-5}$

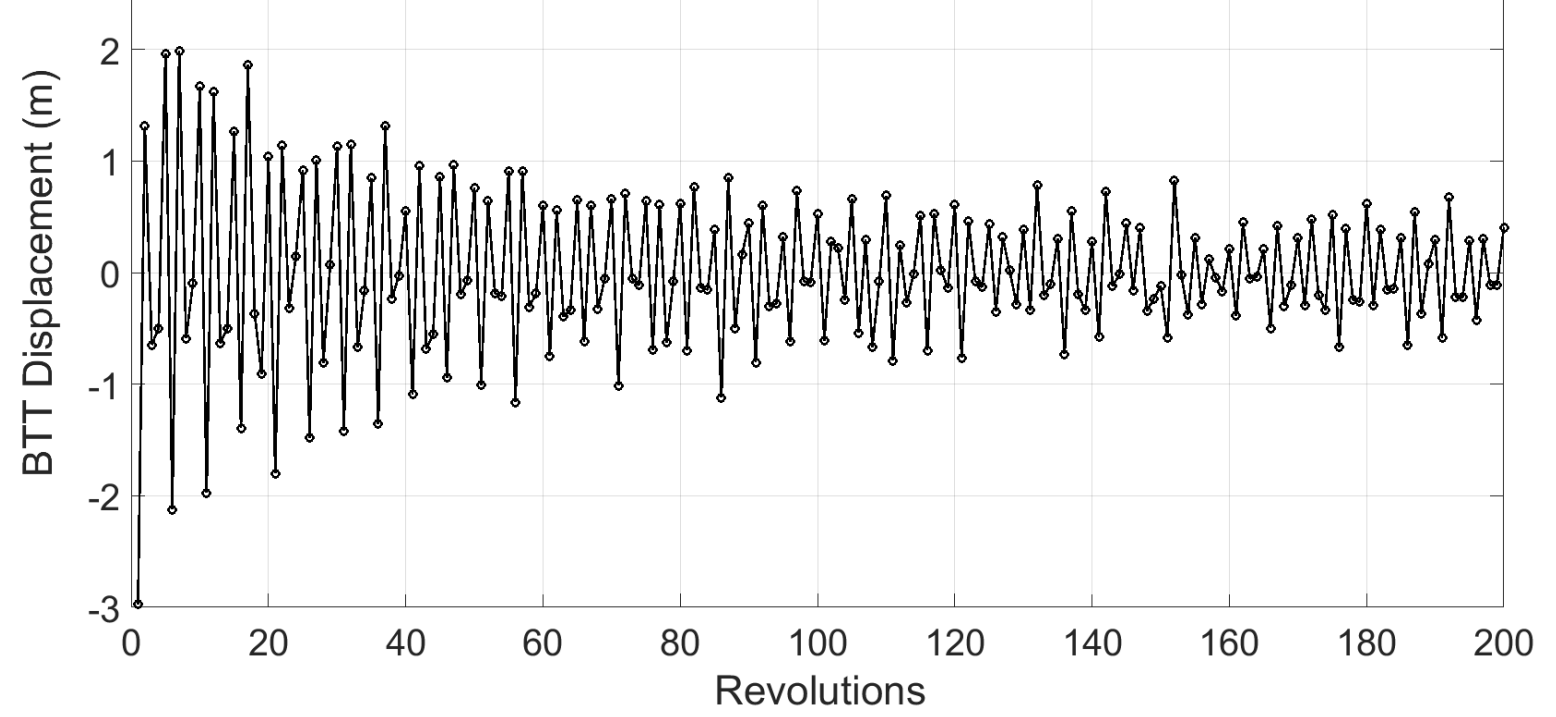

Fig. 5. Extraction of asynchronous component from simulated raw BTT data (2EO $+3.6 \mathrm{EO}+$ noise at $20 \% \mathrm{NSR}$ ) of a given blade at one probe: (a) averaging (without masking) of raw data; (b) extracted 3.6EO component. 
1

2

3

4

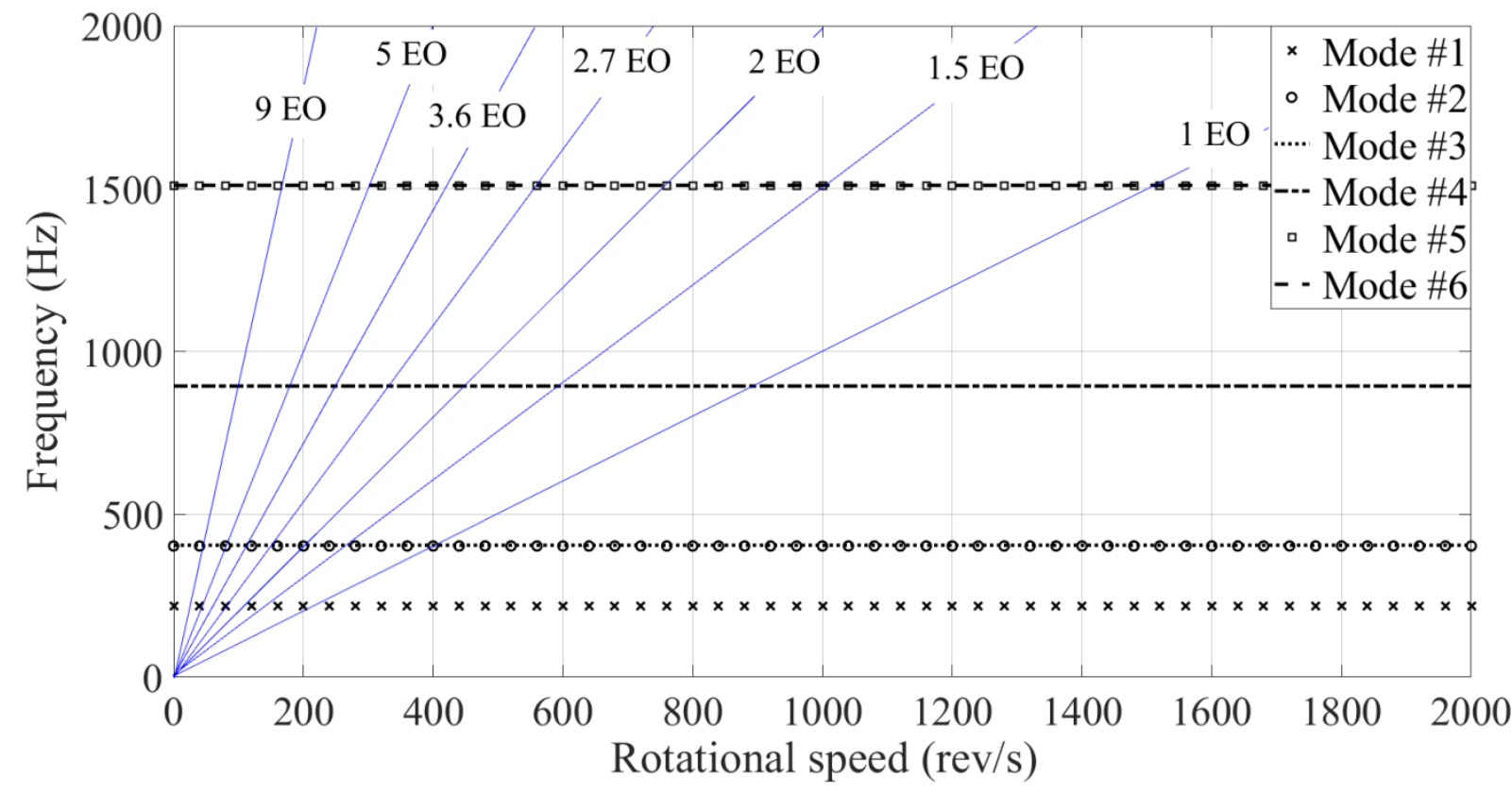

8

Fig. 6. Campbell diagram of the blisk model used in sections (4 and 5). Modal frequencies are invariant with speed since rotational inertia effects are not included in simulator.

9

10

11

12

13

14

15

16

17

18

19 
2

3

4

5

6

7

8

9

10

11

12

13

14

15

16

17

18

19

(a)

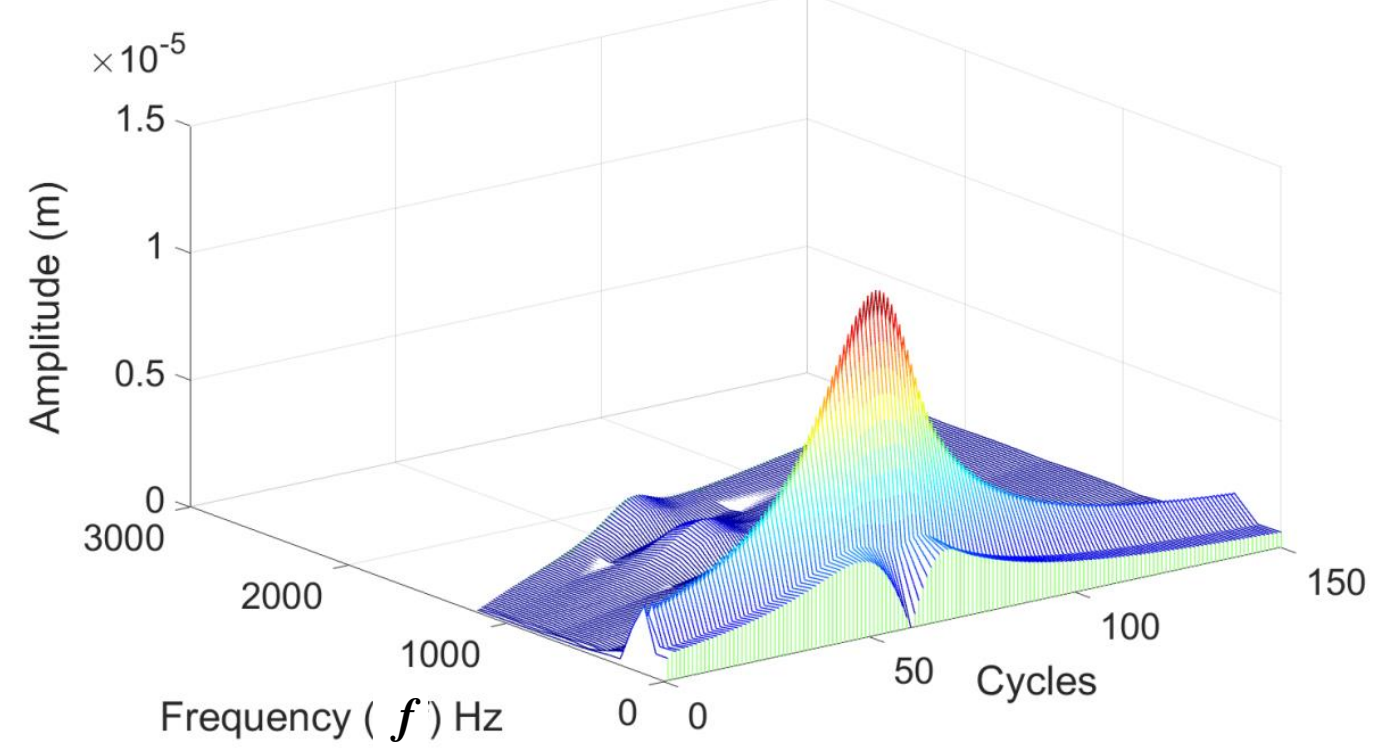

(b)

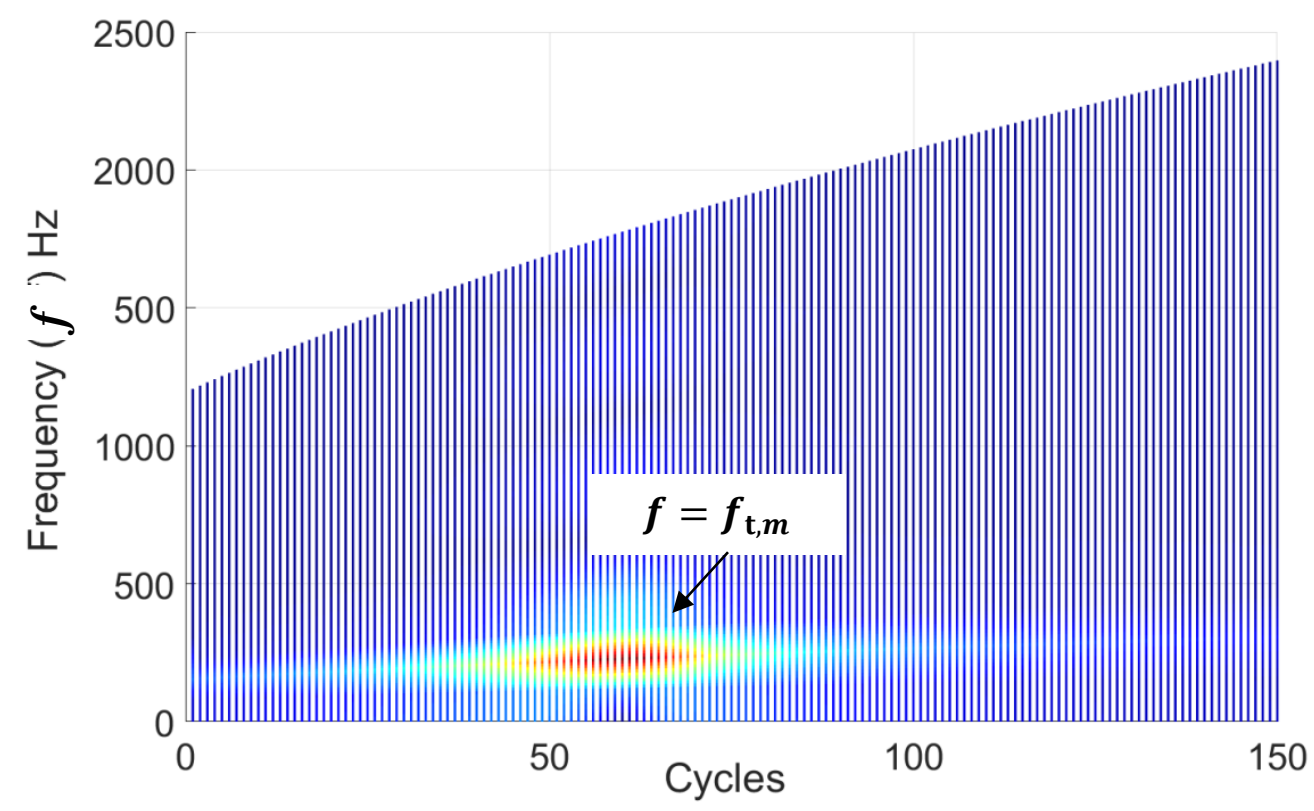

Fig. 7. Determination of non-integral EO from travelling wave pattern of the BTT data of all blades past a given probe: (a) travelling wave plot (isometric view); (b) travelling wave plot (top view) (BTT data is for test 2 in Table 2). 


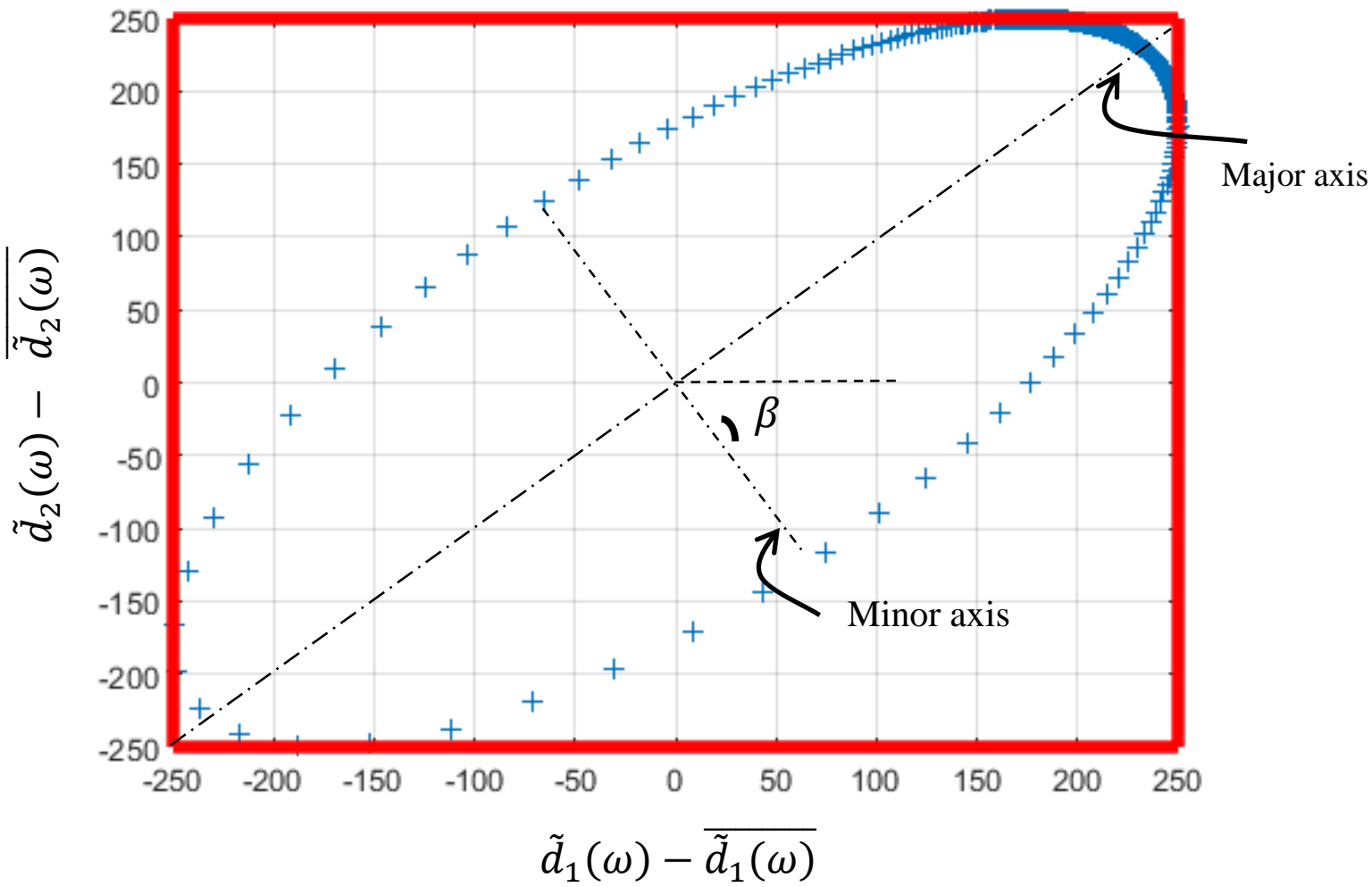

Fig. 8. Elliptical Relation between data from two probes in 2PP method

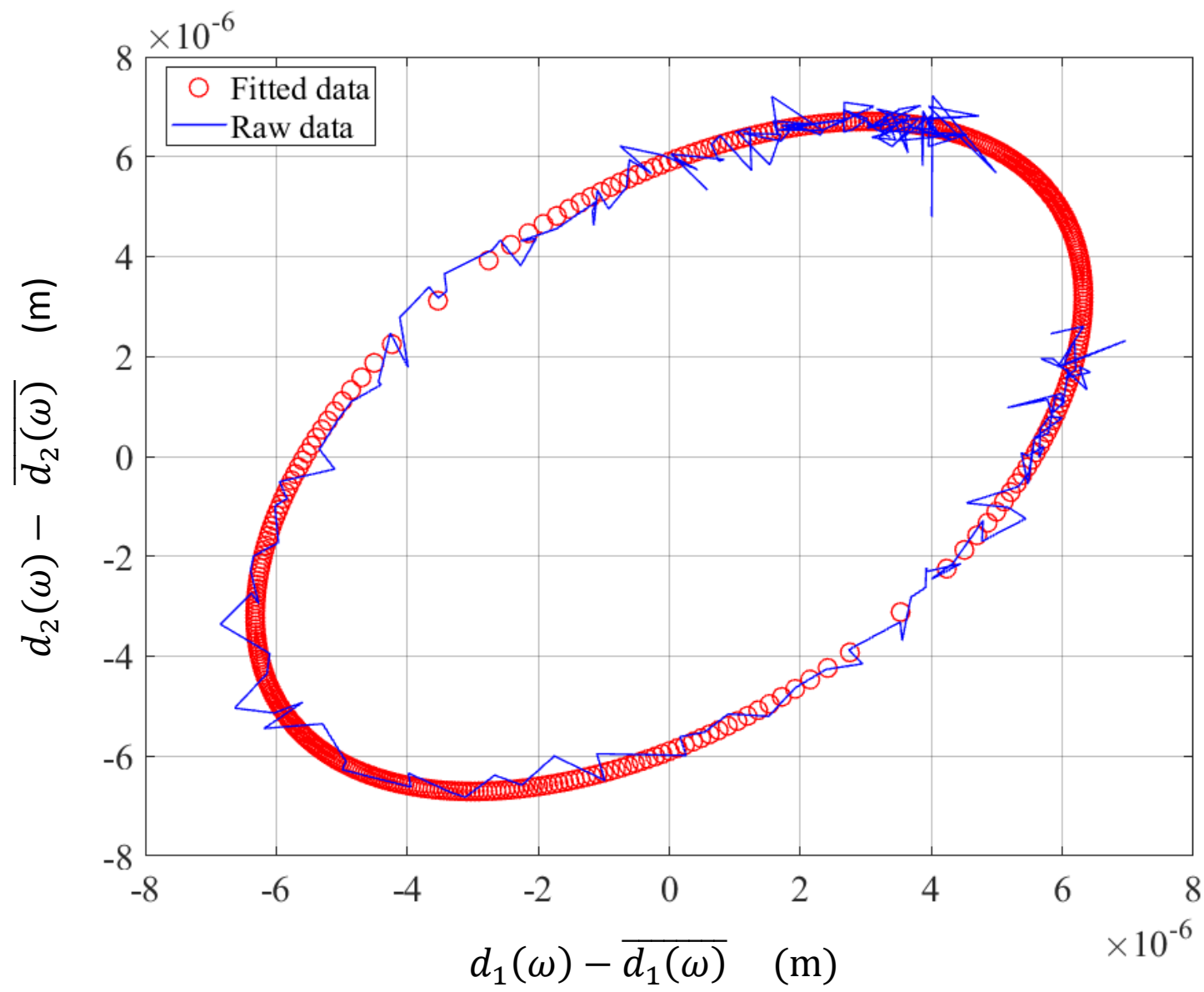

Fig. 9. Two parameter plot of data generated by the simulator of this paper (with added noise of 5\% NSR) 


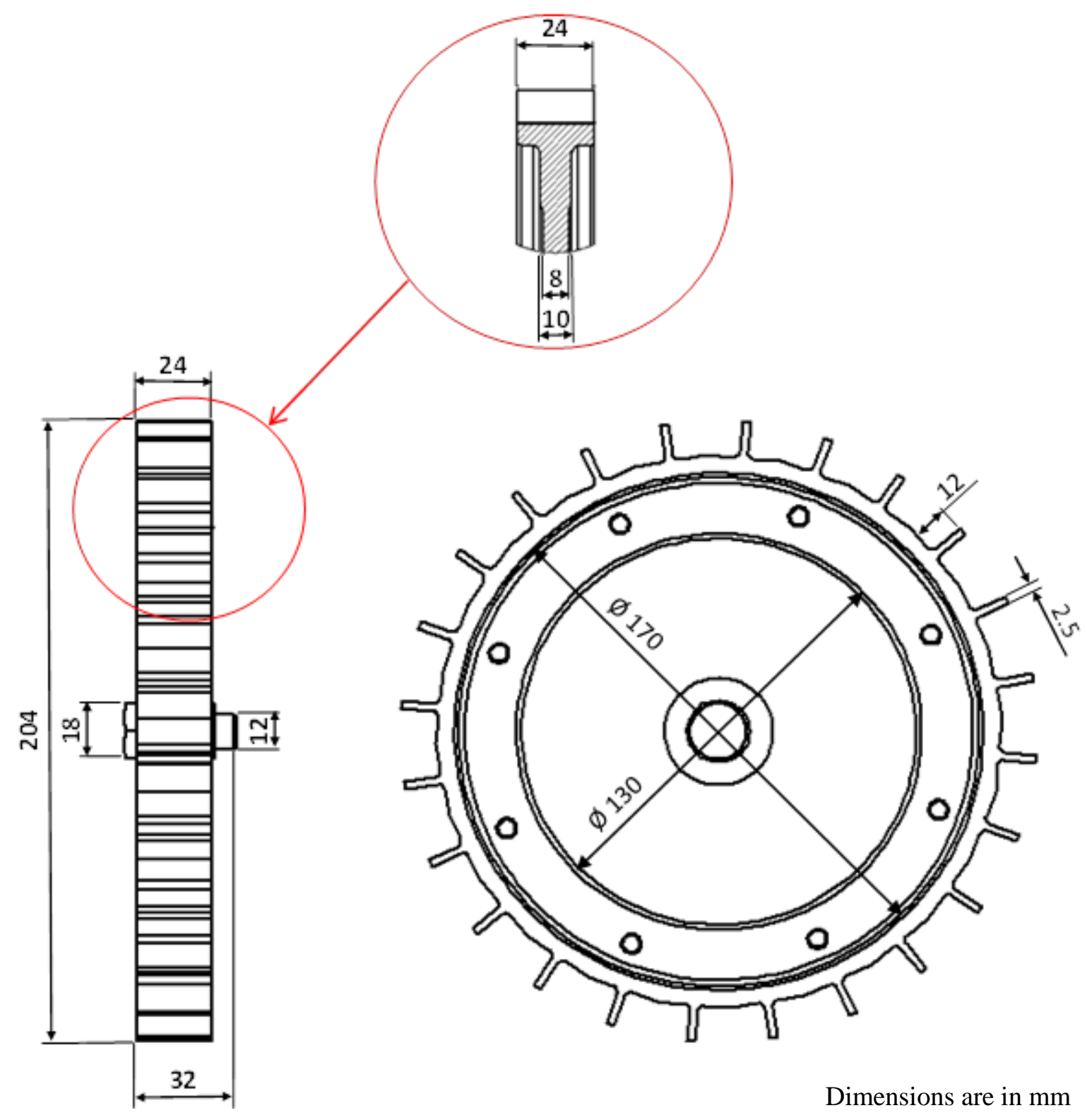

Fig. 10. Detailed blisk design 
Fig. 11. Rig for centrally mounted stationary blisk testing (conf. (2))

13 


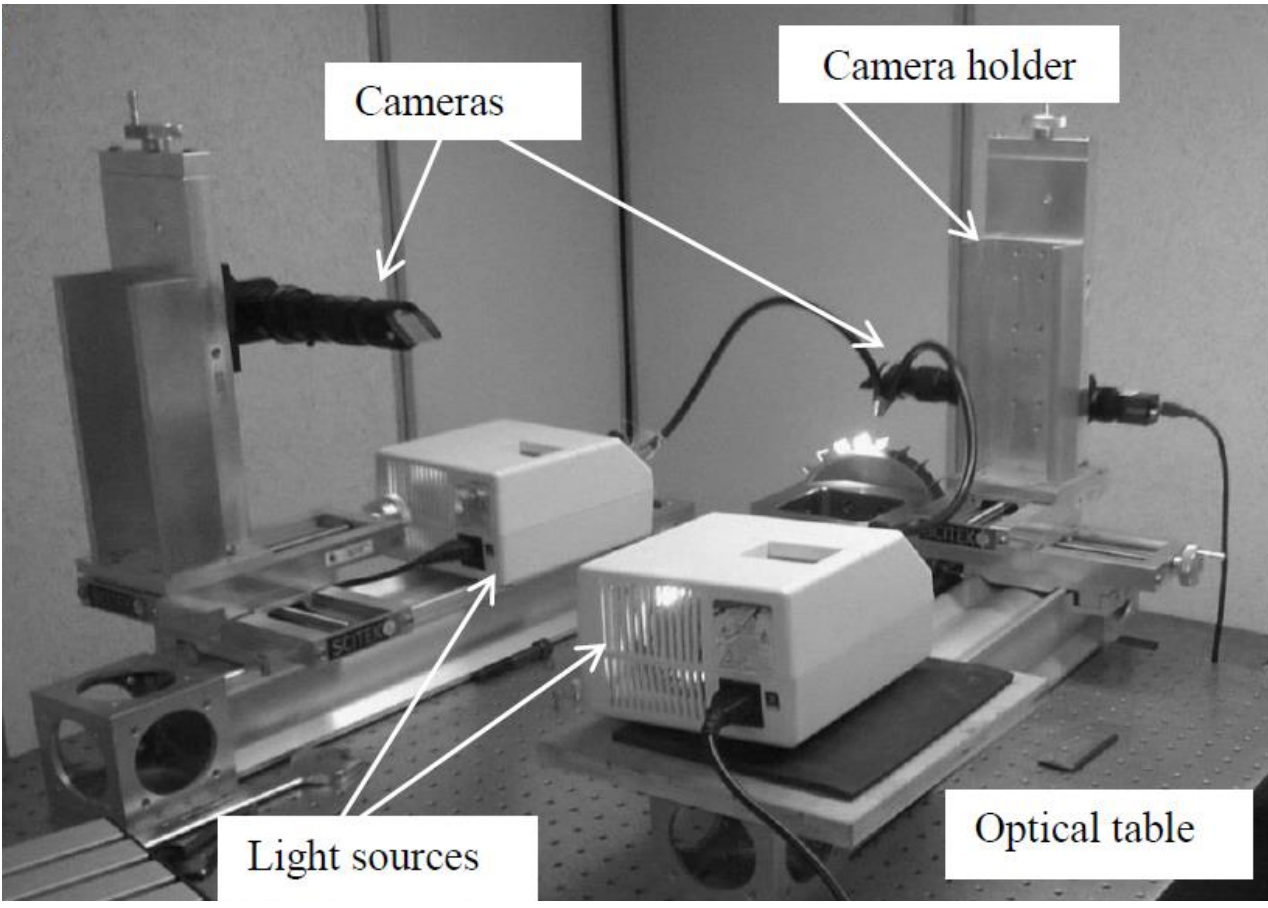

Fig. 12. FireWire cameras measurement system for centrally mounted stationary blisk testing (conf. (2)).

\section{4}

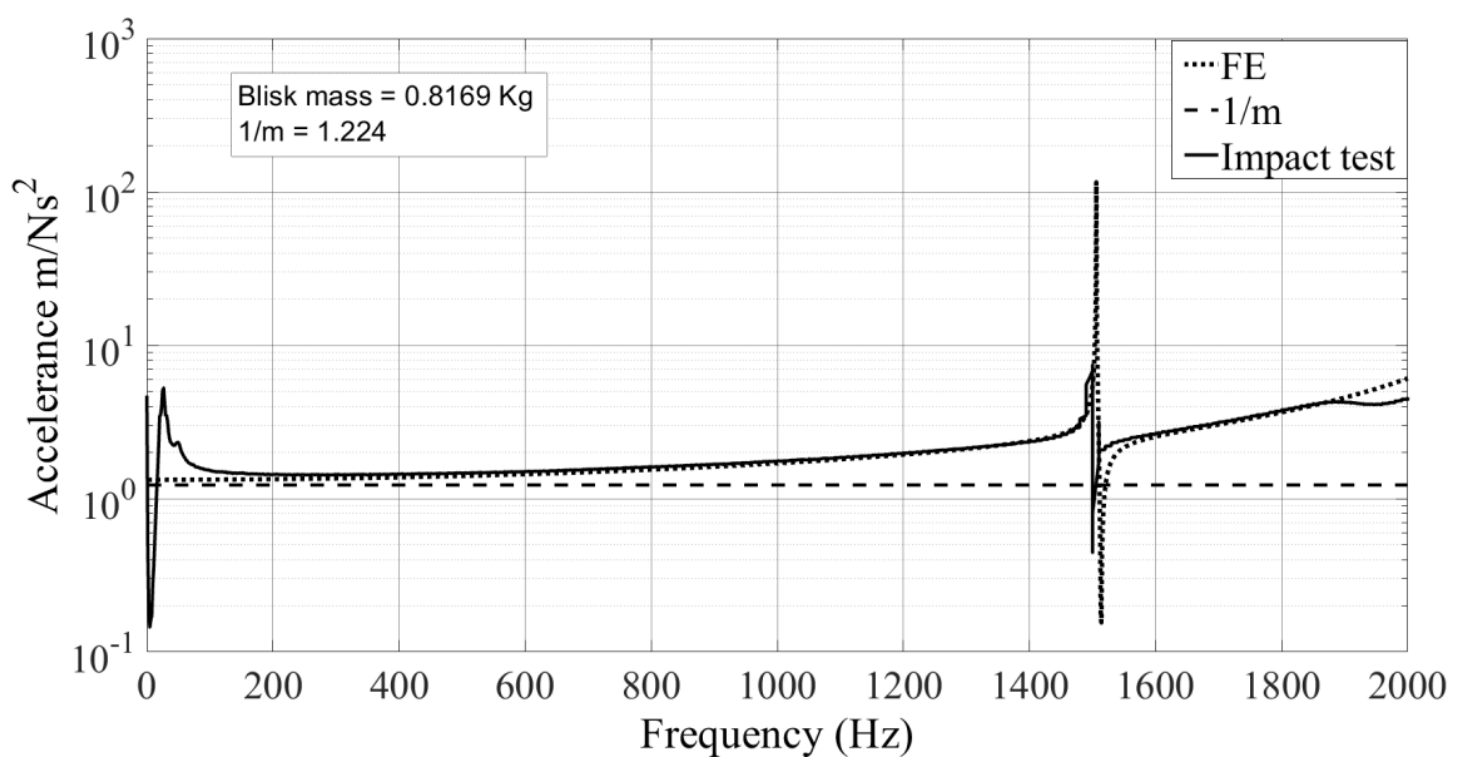

Fig. 13. Impact test result for typical frequency response function (FRF) magnitude of free blisk (conf. (1)) subject to excitation normal to its plane. 


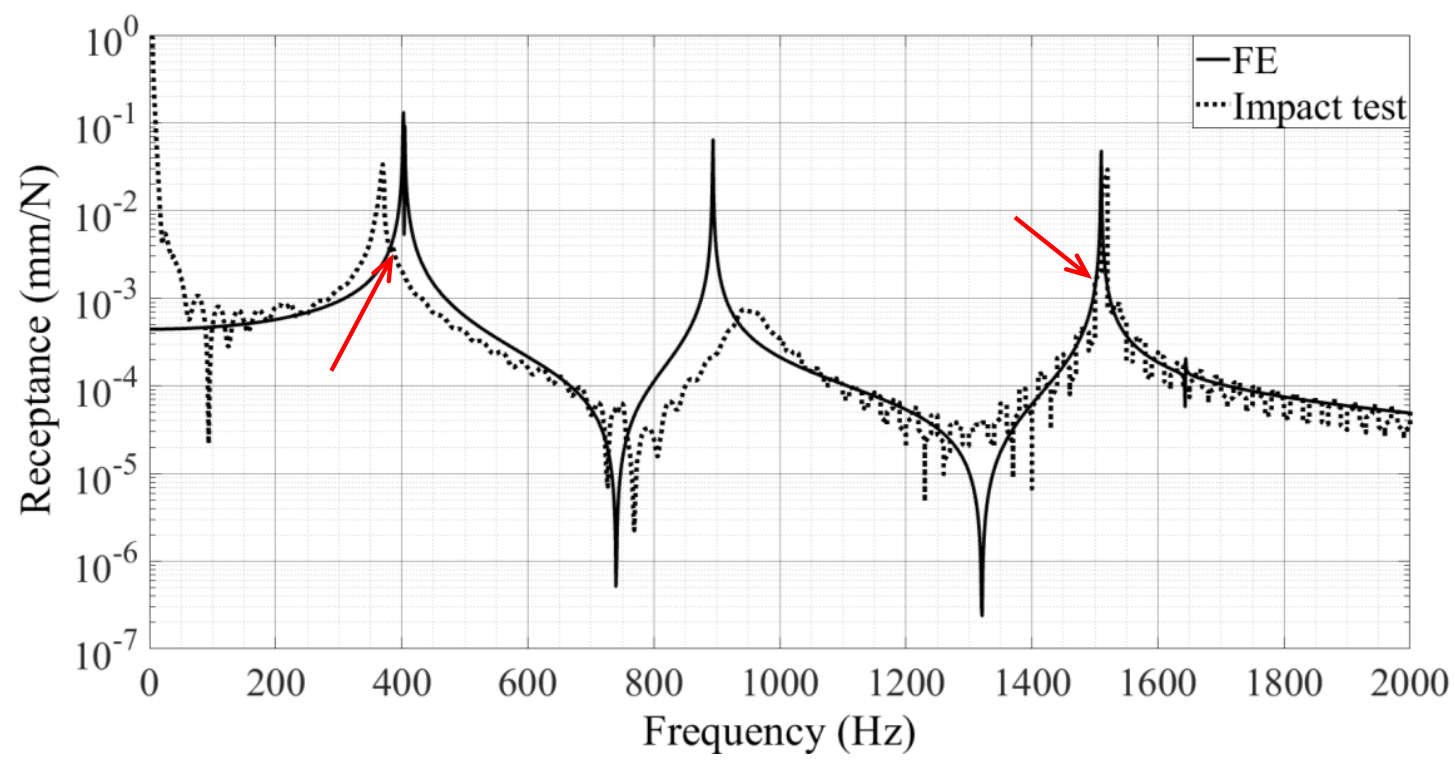

Fig. 14. Impact test result for typical frequency response function (FRF) magnitude of centrally mounted blisk (conf. (2)) subject to excitation normal to its plane (minor, barely visible peaks on measured FRF indicated with arrows).

(a)

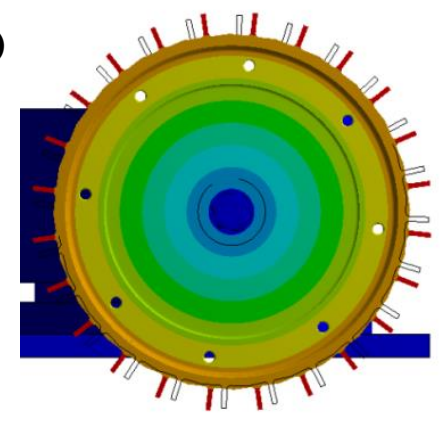

(d)

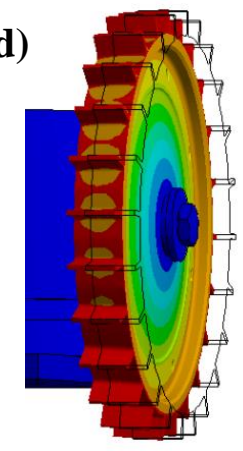

(b)

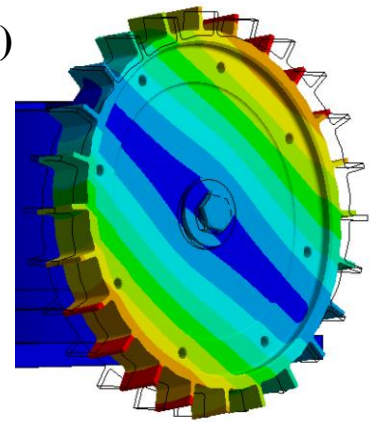

(e)

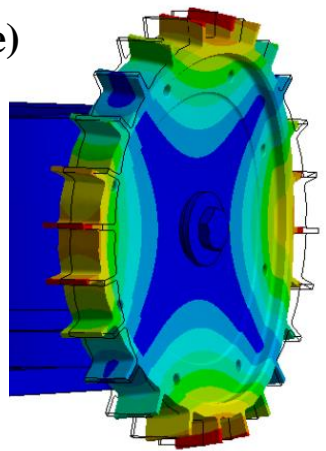

(c)

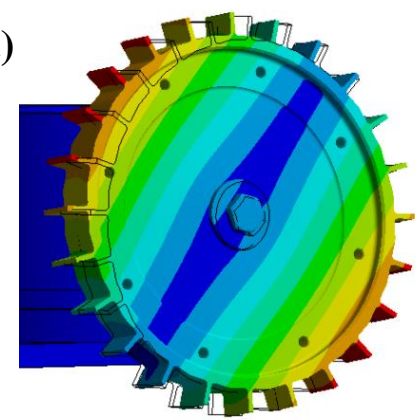

(f)

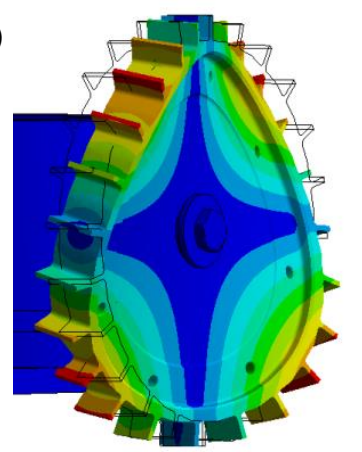

Fig. 15. Predicted mode shapes of the stationary, centrally mounted blisk (conf. (2)): (a) mode 1 $(218.7 \mathrm{~Hz})$; (b) mode $2(402.6 \mathrm{~Hz})$; (c) mode $3(404.7 \mathrm{~Hz})$; (d) mode $4(893.7 \mathrm{~Hz})$; (e) mode 5 $(1509.7 \mathrm{~Hz})$; (f) mode $6(1509.9 \mathrm{~Hz})$. (please refer to online version for more clarity). 
(a) clarity).

16

7

8

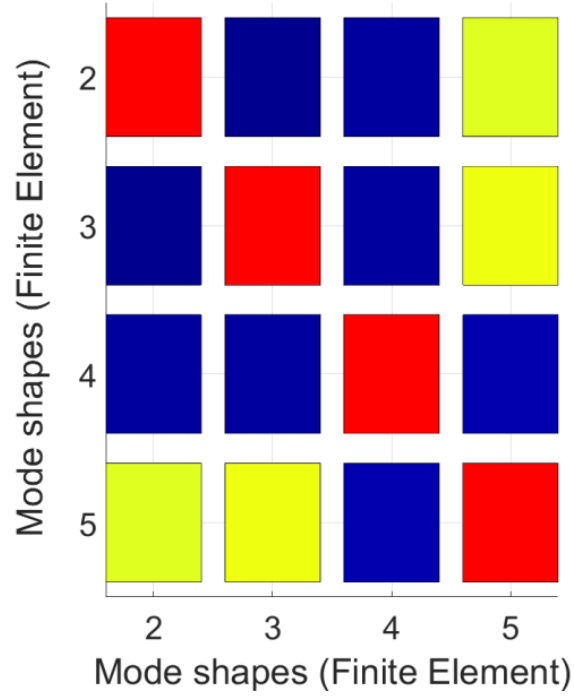

(b)

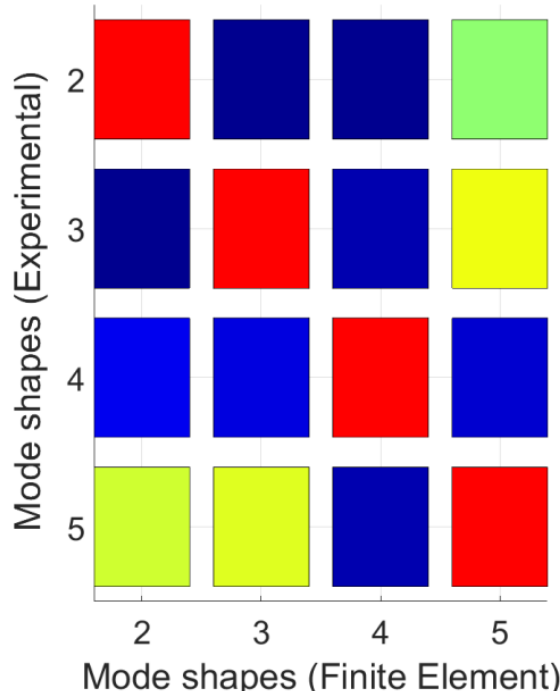

Fig. 16. Modal assurance criteria (MAC) matrices of modes 2 to 5 of stationary, centrally mounted blisk (conf. (2)): (a) Auto MAC matrix for FE-predicted mode shapes; (b) cross MAC matrix relating experimental mode shapes with FE-predicted mode shapes (please refer to online version for more 
(a)

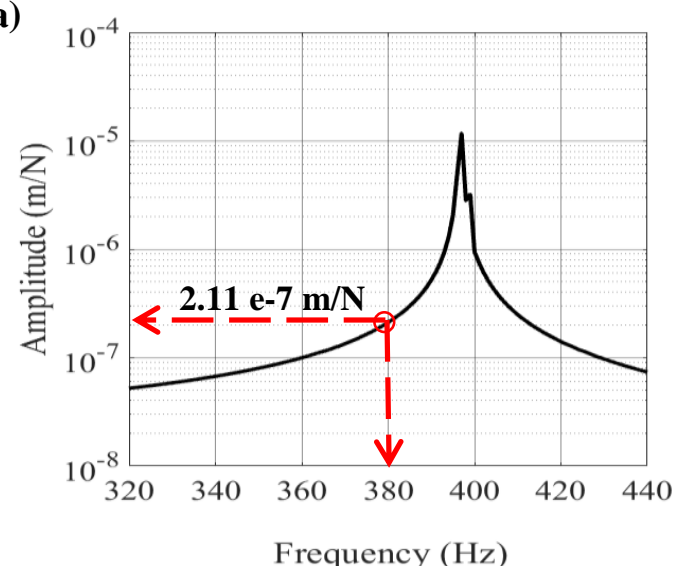

(c)

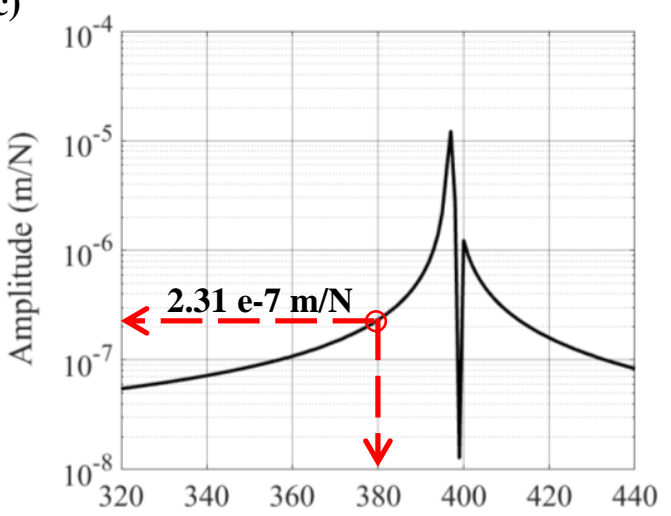

Frequency $(\mathrm{Hz})$

(e)

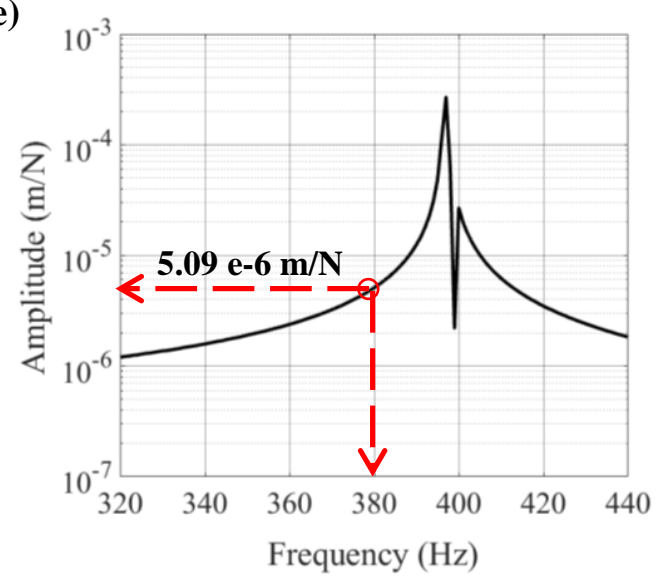

(b) $4 \times 10^{-7}$

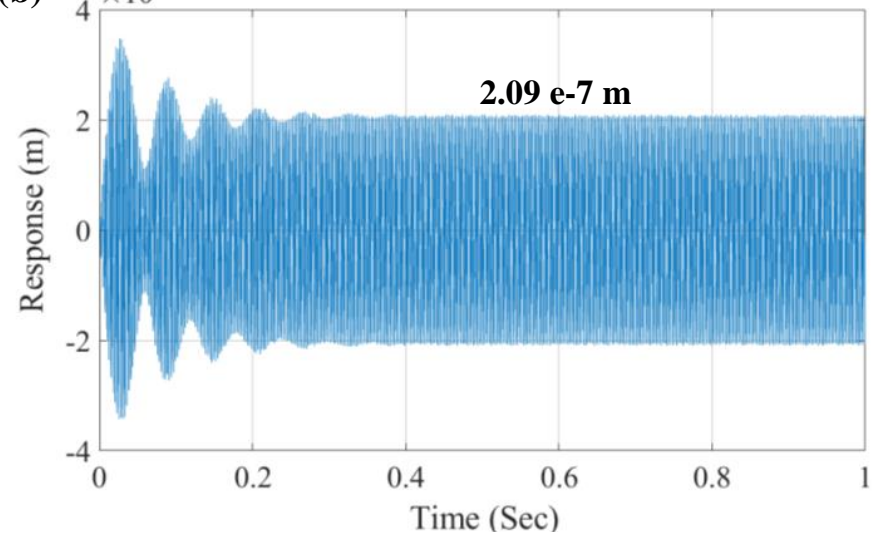

(d)

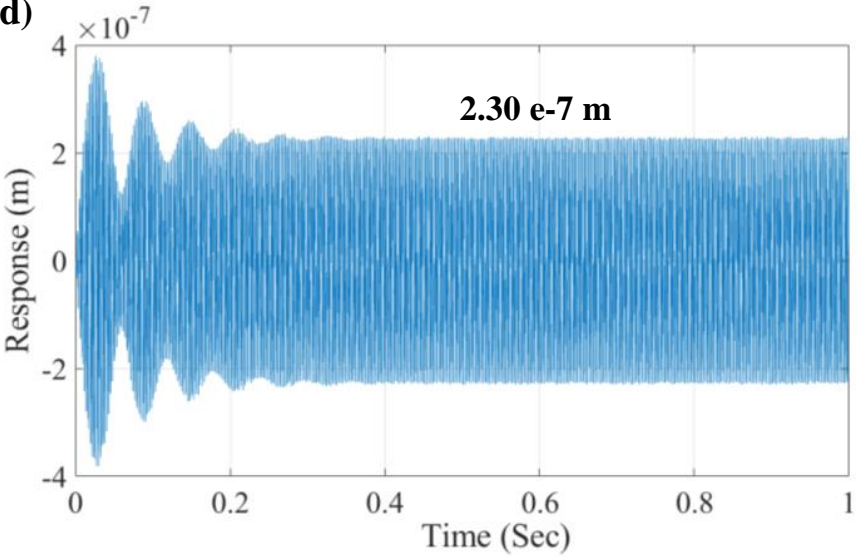

(f)

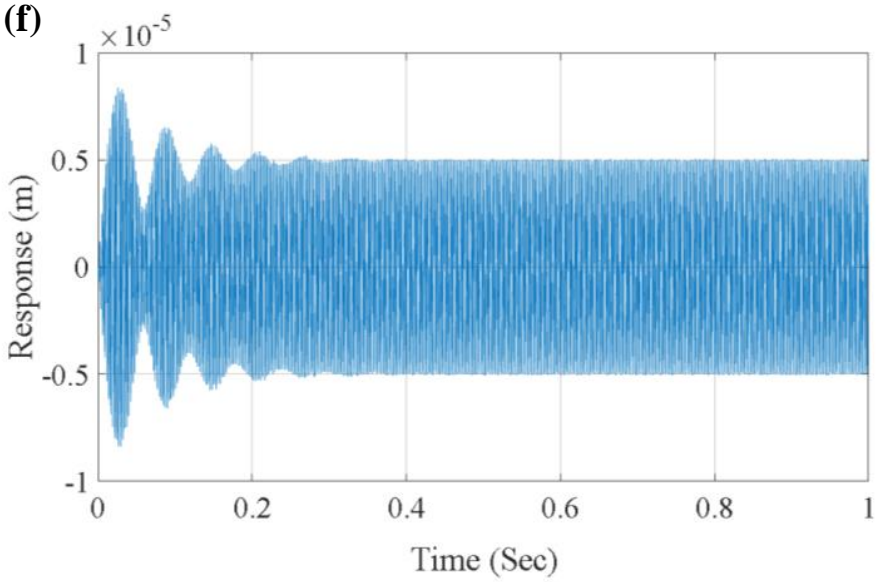

Fig.17. Convergence of modal transformation with six modes for time domain dynamic deflection of centrally mounted blisk, for harmonic excitation in $z$-direction (unit amplitude, $380 \mathrm{~Hz}$ ) and response at a typical blade tip: (a) exact frequency response function (FRF) from FE for response in $x$-direction; (b) simulator time domain (TD) response ( $\boldsymbol{x}$-component of $\left.\boldsymbol{\Delta} \mathbf{u}^{(\mathrm{def})}(\boldsymbol{t})\right)$; (c) FE FRF response ( $y$-direction); (d) simulator TD response ( $\boldsymbol{y}$-component of $\left.\boldsymbol{\Delta} \mathbf{u}^{(\text {def })}(\boldsymbol{t})\right)$; (e) FE FRF response (z-direction); (f) simulator TD response ( $\mathbf{z}$-component of $\left.\Delta \mathbf{u}^{(\text {def })}(\boldsymbol{t})\right)$. 
(a) $4 \times 10^{-5}$

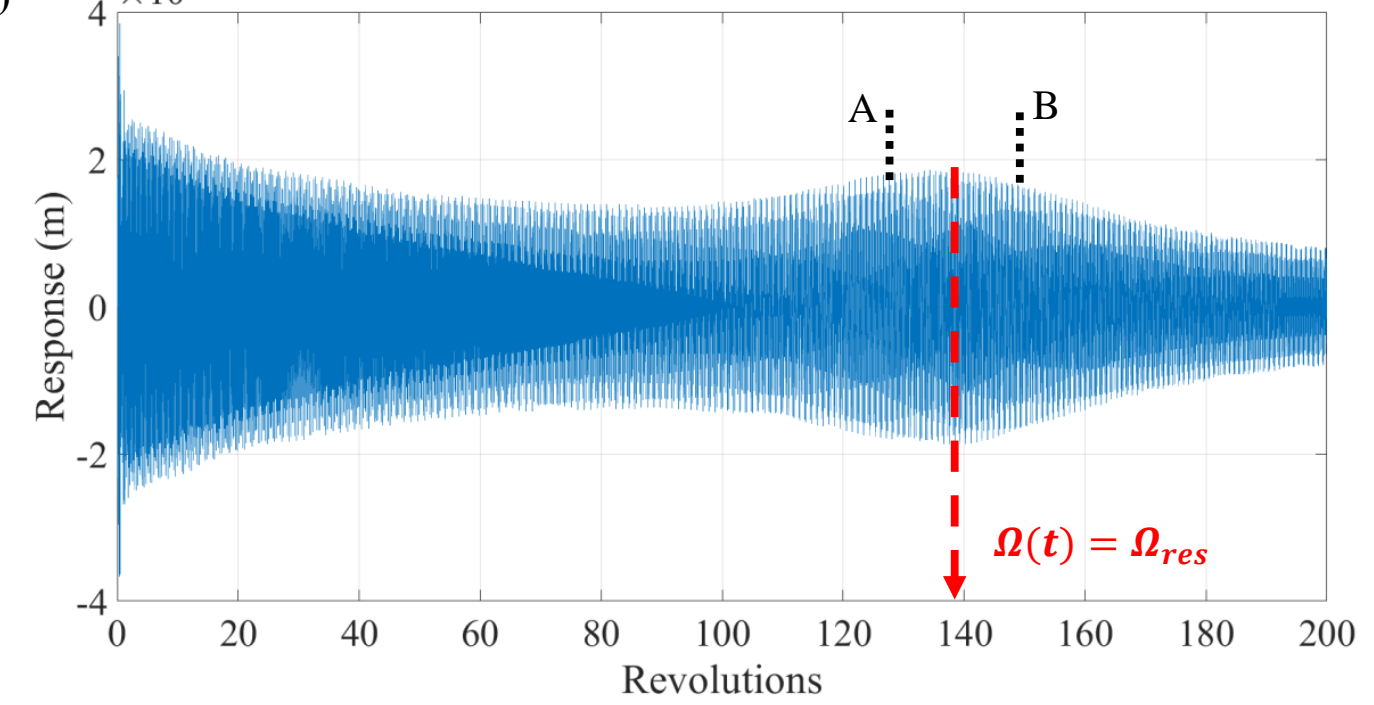

(b) $\quad 4 \times 10^{-5}$

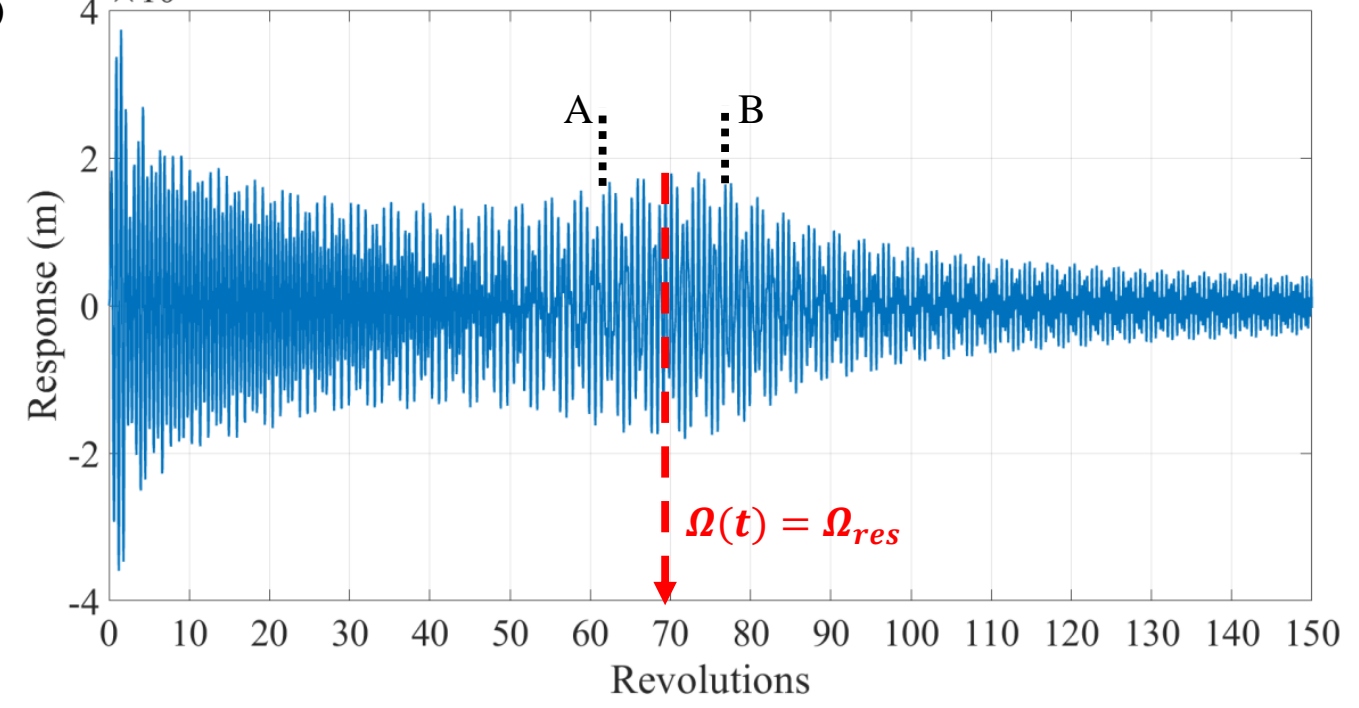

(c) $\quad 4 \times 10^{-5}$

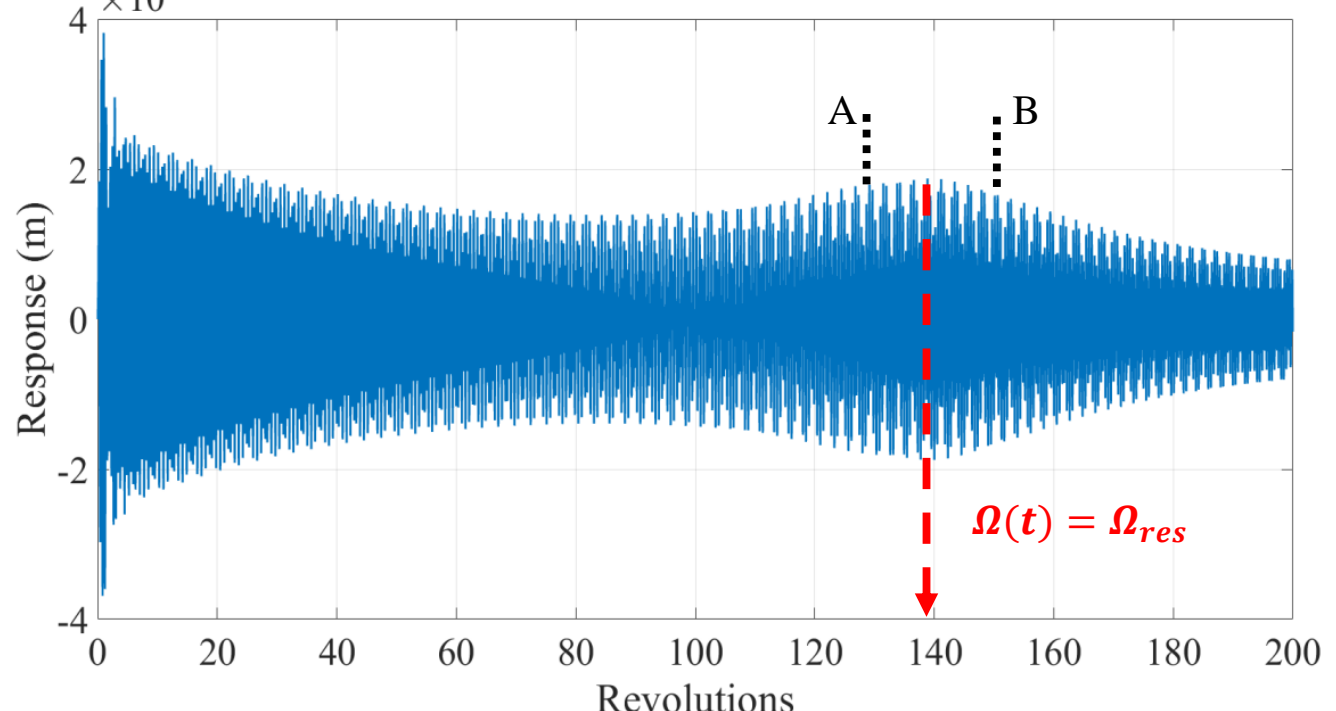

Fig. 18. The tangential vibratory responses calculated from $\Delta \mathbf{u}^{(\mathrm{def})}(t)$ of centrally mounted blisk at the nominal sensing point of a given blade tip: (a) test 3, (b) test 4, (c) test 5 . Positions of resonance indicated with red arrows. A and B are the boundapies of the data set used in FFT (section 5.2). 


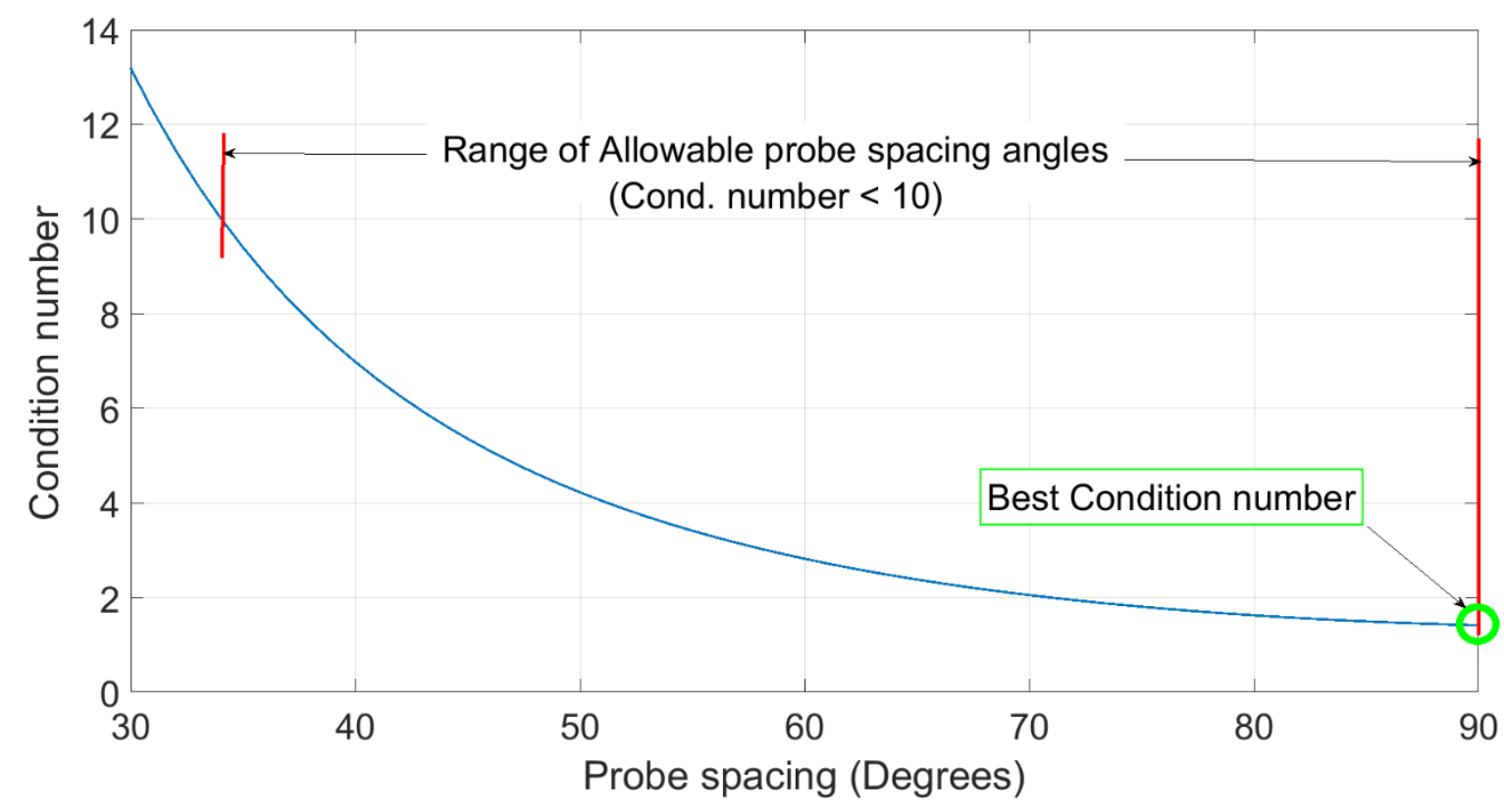

Fig. 19. Determination of allowable probe spacing for MSFP method $\left(\mathrm{EO}=1, N_{\mathrm{Pr}}=4\right)$

13

14

15

16

17

18

19

20

21

22

23

24

25

26

27 


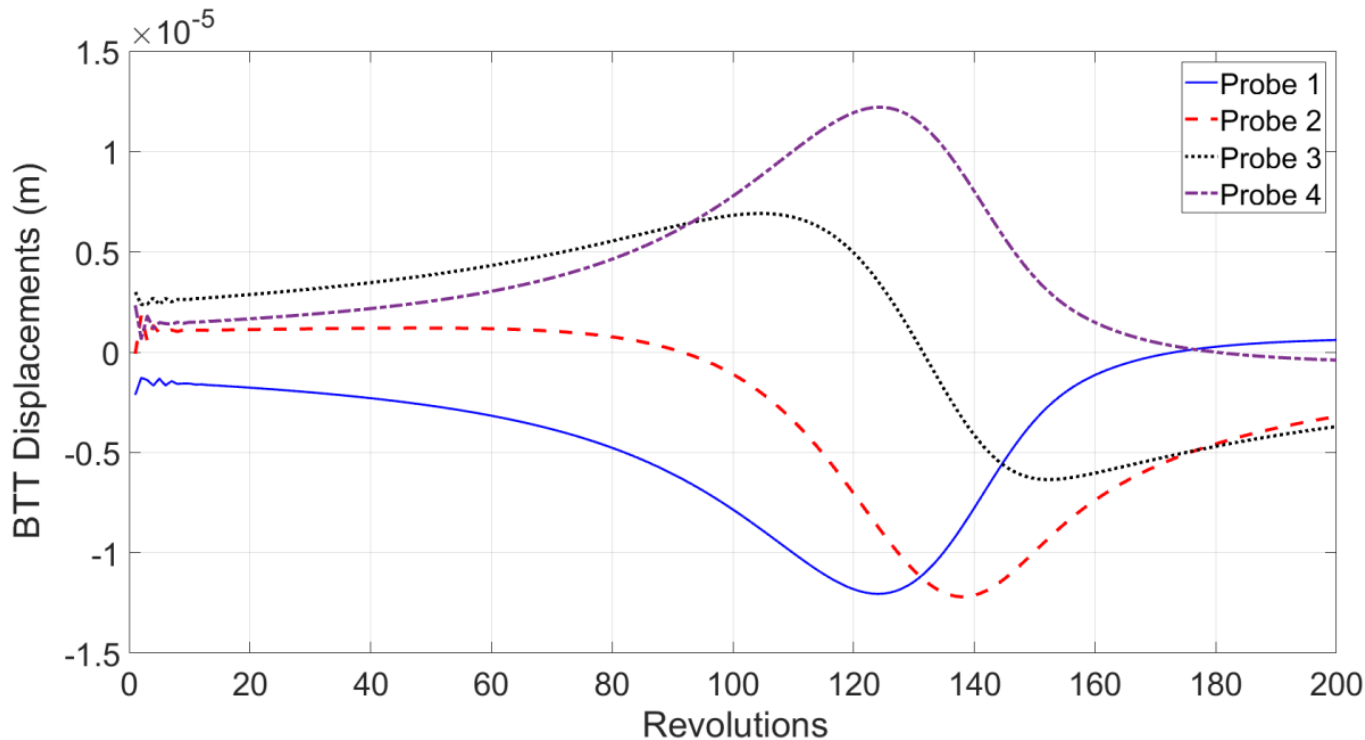

Fig. 20. Simulated BTT data of given blade at probes 1-4 prior to addition of noise for test 1 , Table 2 (1EO) (probe angles for test 1 (MSFP, MGARES, 2PP), Table 3).
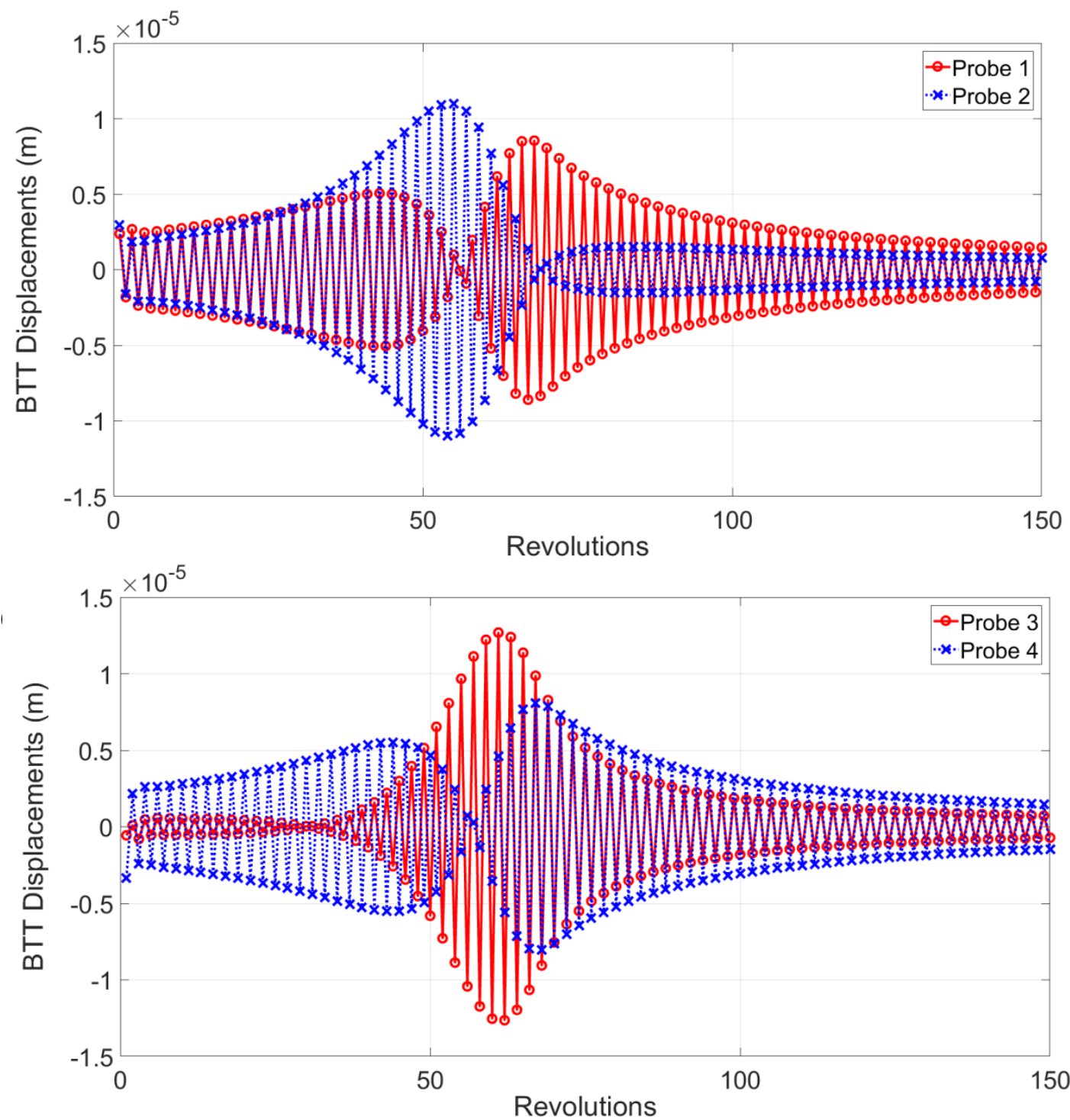

Fig. 21. Simulated BTT data of given blade at probes 1-4 prior to addition of noise for test 2 , Table 2 (1.5EO): (a) probes 1, 2; (b) probes 3, 4 (probe angles for test 2 (MSFP, MGARES), Table 3). 


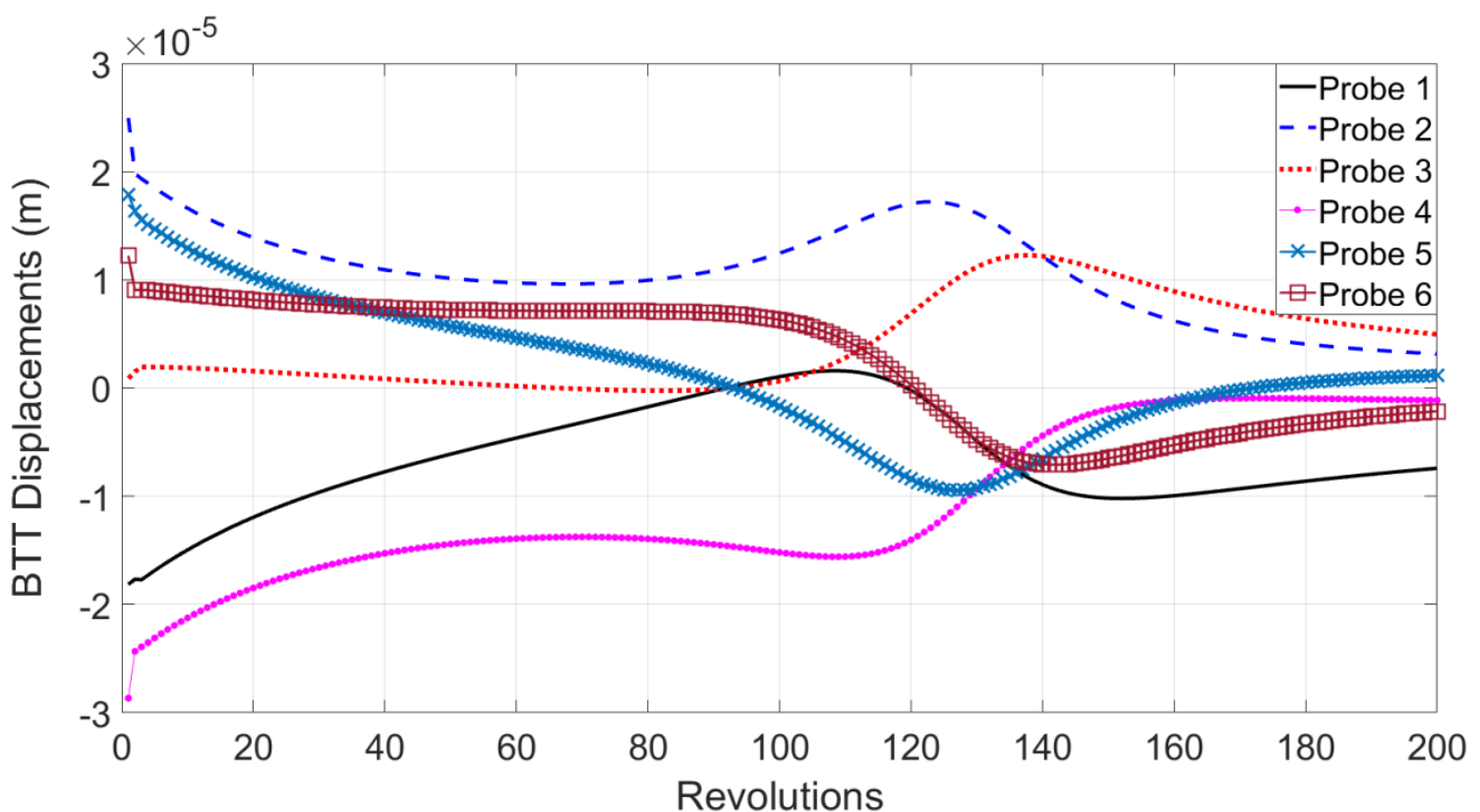

Fig. 22. Simulated BTT data of given blade at probes 1-6 prior to addition of noise for test 3 , Table 2 (5EO+9EO) (probe angles for test 3 MSFP, Table 3). 


列

1
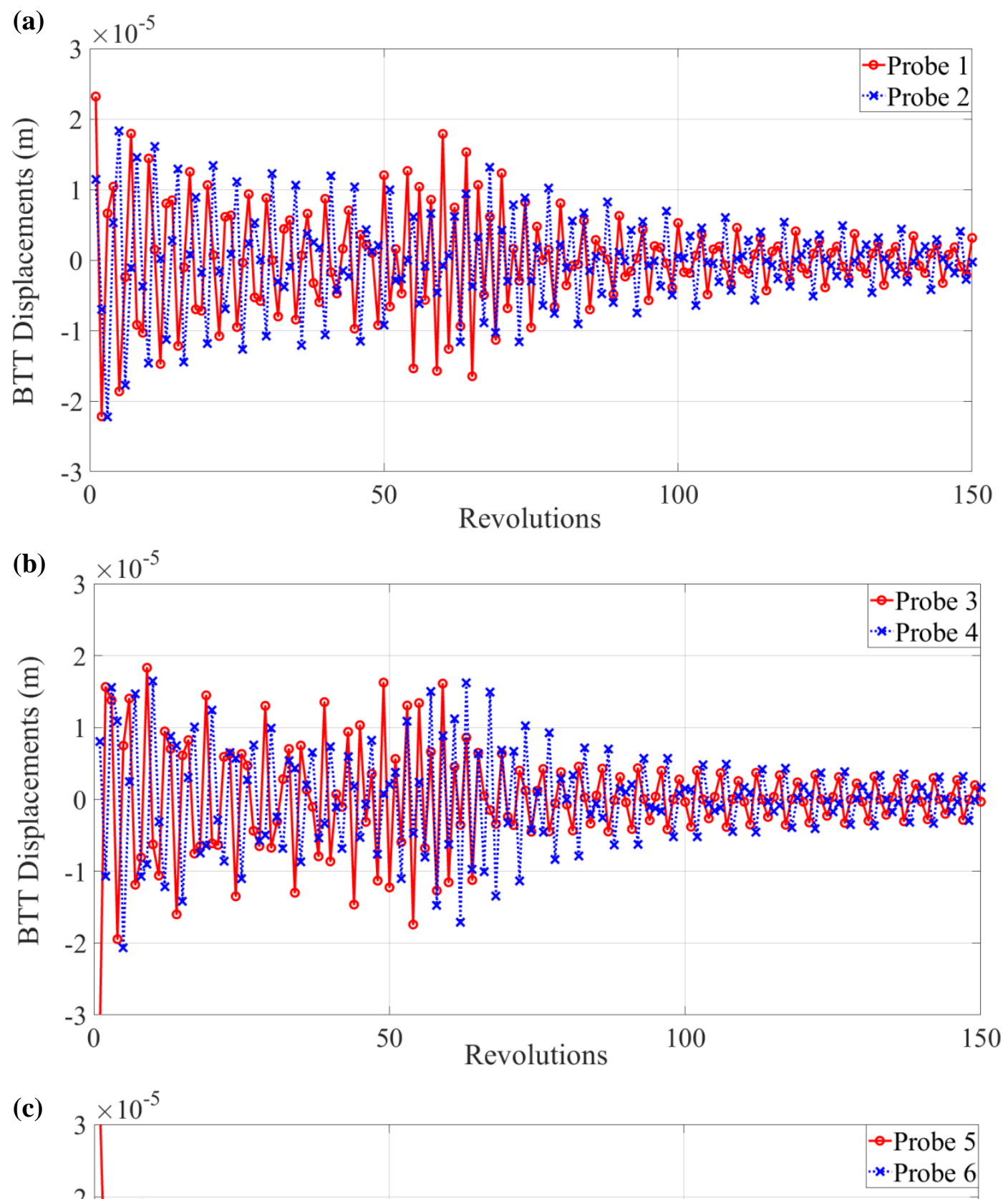

2

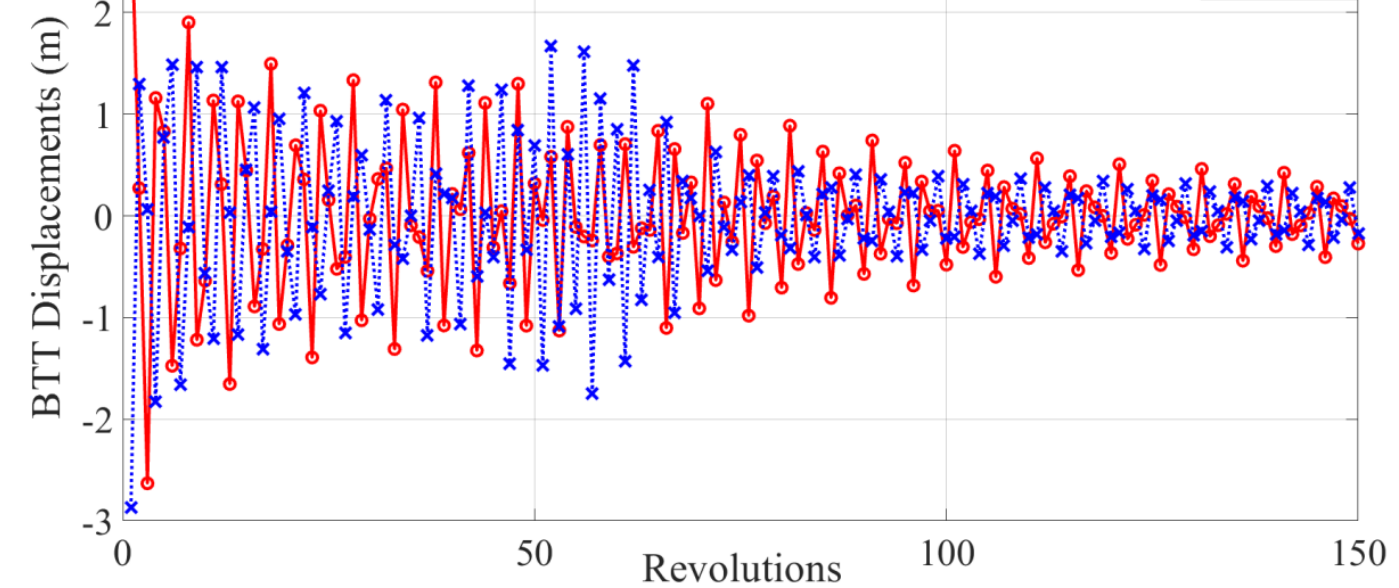

Fig. 23. Simulated BTT data of given blade at probes 1-6, prior to addition of noise for test 4 , Table 2 (1.5EO+2.7EO): (a) probes 1, 2; (b) probes 3,4; (c) probes 5,6 (probe angles for test 4 MSFP, Table 3). 
1

2

3

4

5

6

7

8

9

10

11

12

13

14

15

16

17

18

19

20

21

22

23

24

25

26

27

(a) $3 \times 10^{-5}$

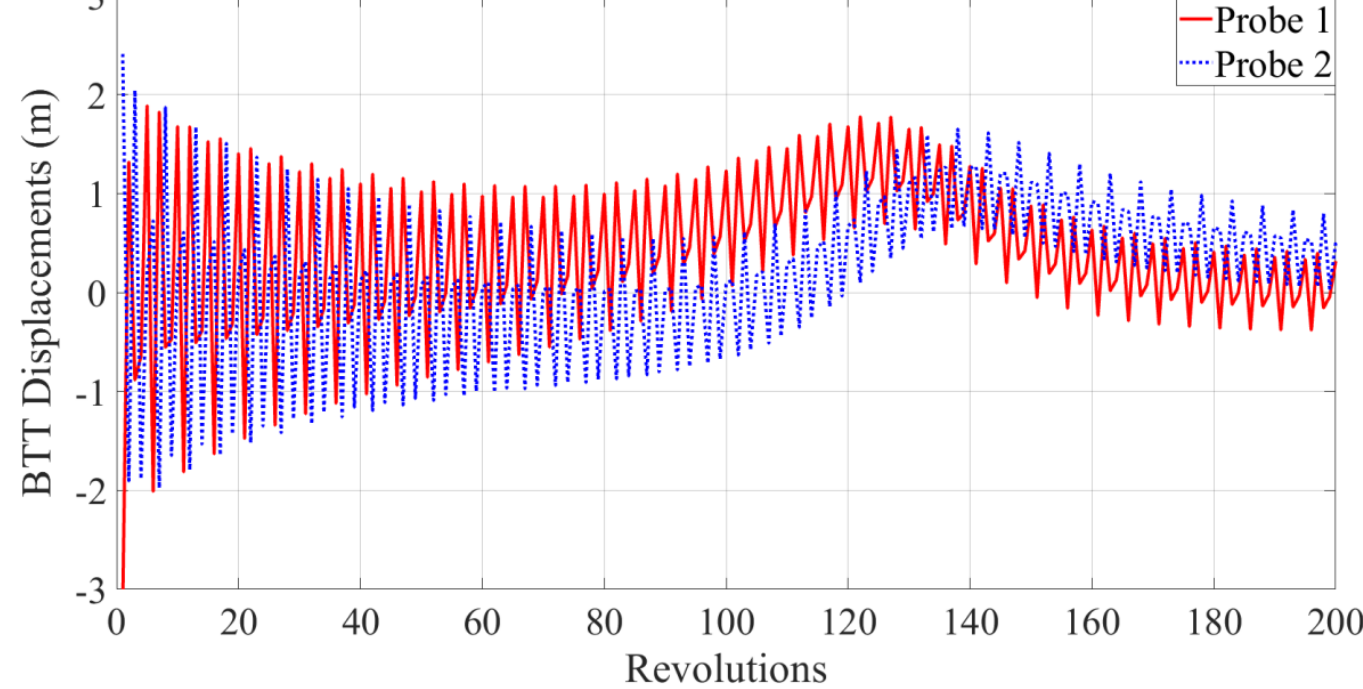

(b) $3 \times 10^{-5}$

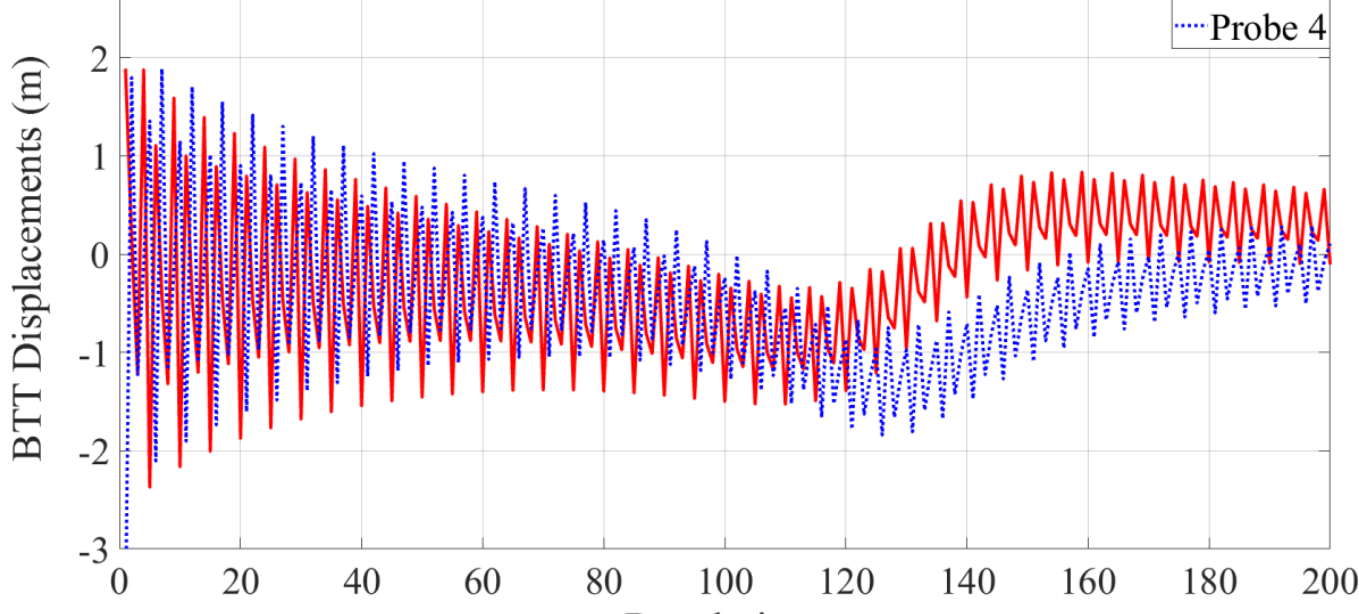

Revolutions

(c) $3 \times 10^{-5}$
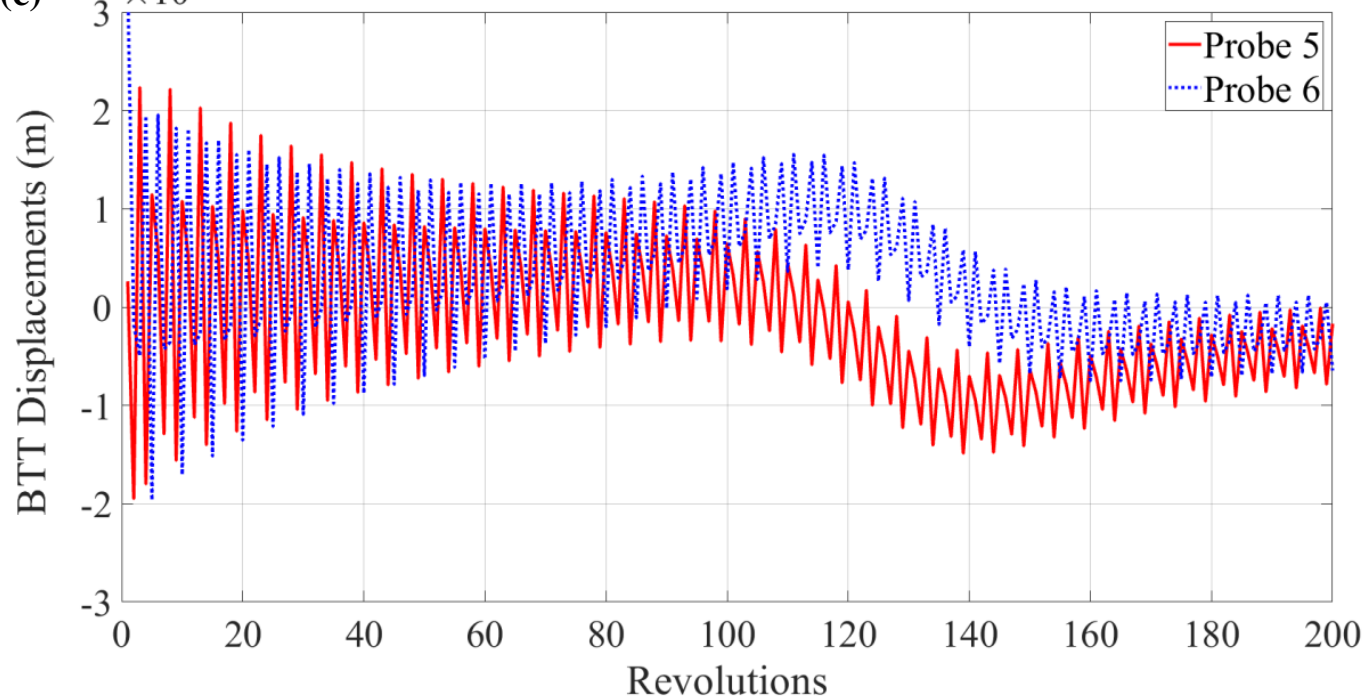

Fig. 24. Simulated BTT data of given blade at probes 1-6, prior to addition of noise for test 5 , Table 2 (2EO+3.6EO): (a) probes 1, 2; (b) probes 3,4; (c) probes 5,6 (probe angles for test 5 MSFP, Table 3). 
1

2

3

4

5

6

7

8

9

10

11

12

13

14

15

16

17

18

19

20

21

22

23

24

25

26

27

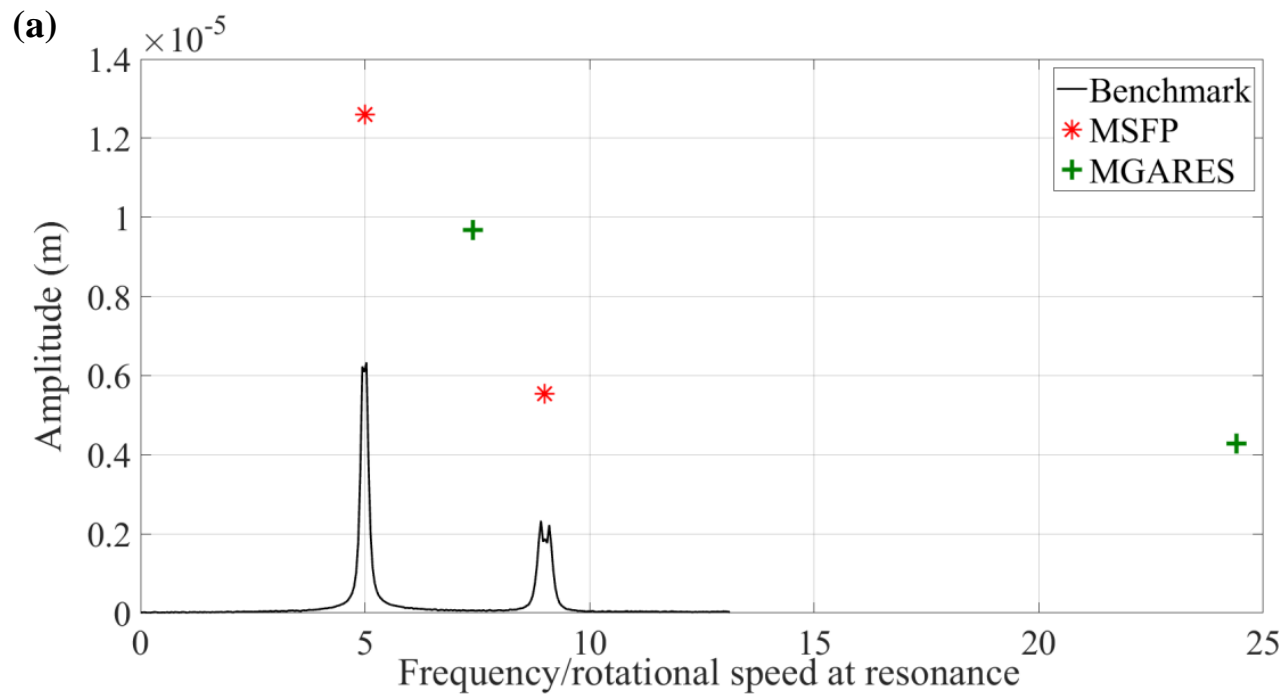

(b)

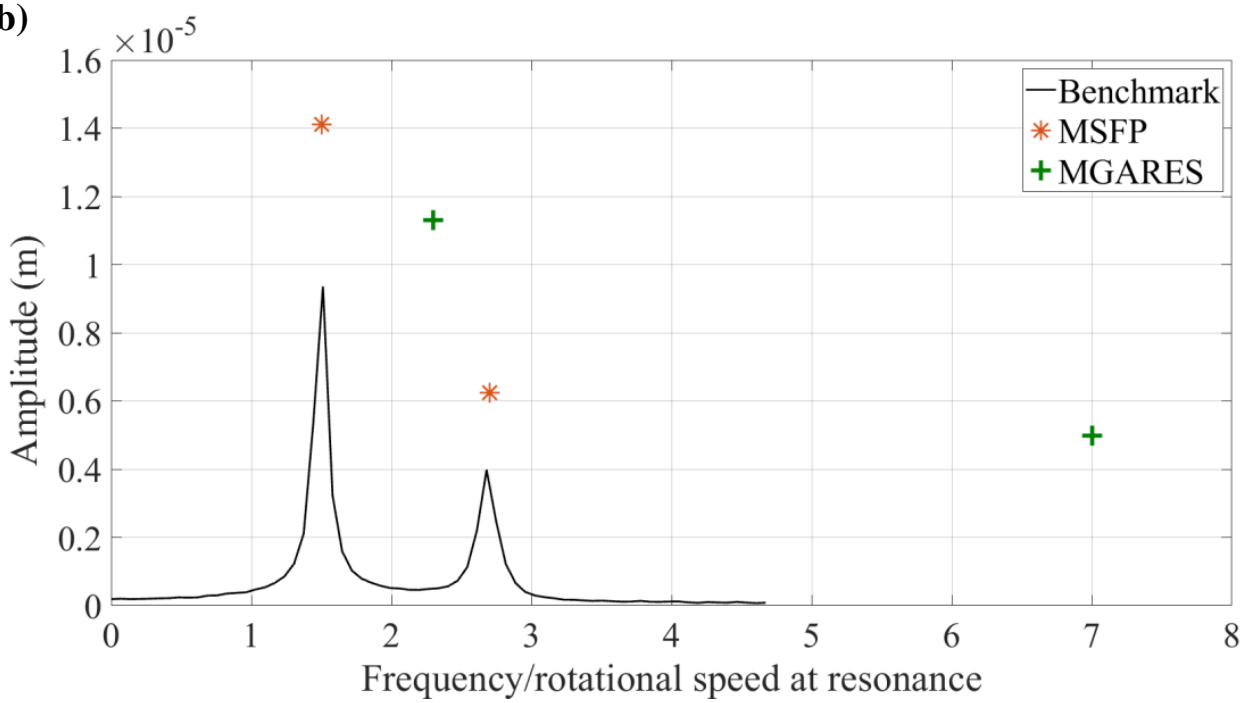

(c)

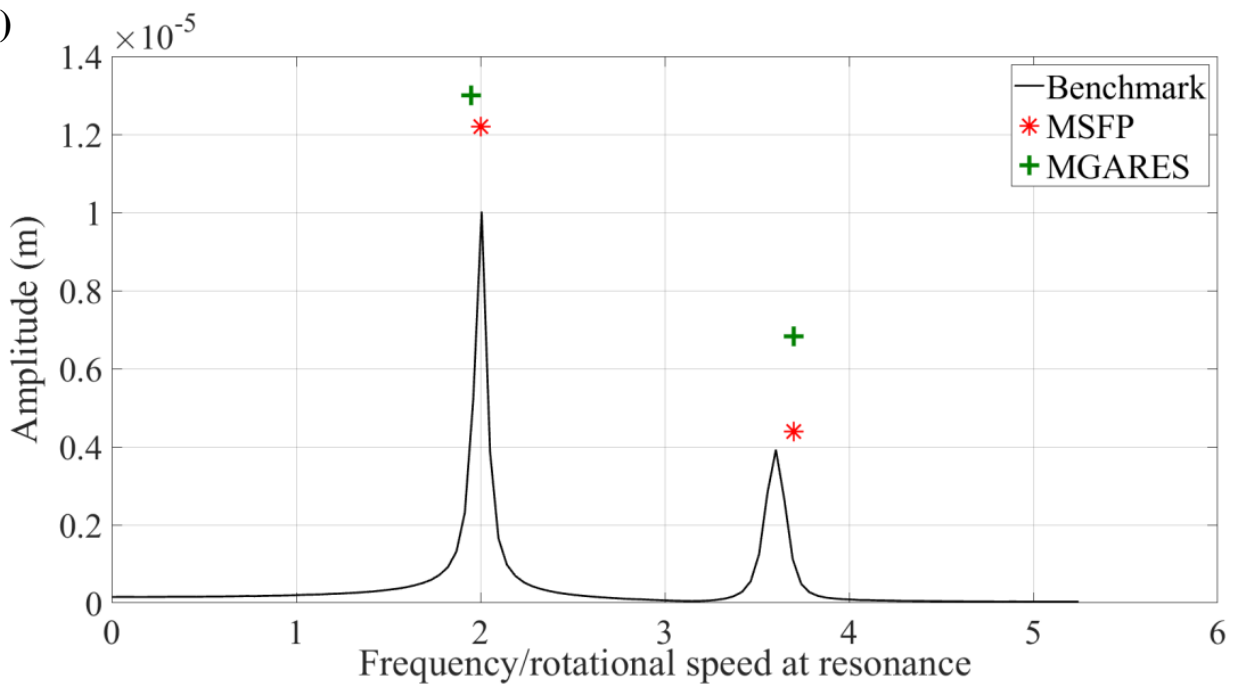

Fig. 25. The estimated BTT frequencies and amplitudes vs the FFT of a short-duration dataset of the benchmark signal centred around the resonance point $\Omega(t)=\Omega_{\text {res }}$ of the speed-sweep response (Fig. 17(a-c)). (a) test 3, (b) test $4,(c)$ test 5. 